\title{
Canadian Spine Society Abstracts
}

18th Annual Scientific Conference

The Fairmont Banff Springs Banff, Alberta

Feb. 28-Mar. 3, 2018 
The Canadian Spine Society is a collaborative organization of spine surgeons advancing excellence in research, education and patient care.

Accreditation: This event is an Accredited Group Learning Activity (Section 1) as defined by the Maintenance of Certification Program of the Royal College of Physicians and Surgeons of Canada, approved by The Canadian Orthopaedic Association.

Course Objectives: The Annual Scientific Conference of the Canadian Spine Society offers a comprehensive review of spine therapy and spine surgery in Canada. The meeting is held in conjunction with the Canadian Paediatric Spine Society and offers current perspectives on the management of both adult and pediatric spinal problems. The program covers both surgical and nonoperative answers to a wide range of spinal pathologies and addresses the more general issues of pain and pain management. It provides practical instruction for the treatment of specific common clinical challenges but also reports on advances in basic spine research and contemporary philosophies on the patient-centered approach. The conference includes the yearly review of work by the Canadian Spine Outcomes and Research Network (CSORN) and will include both papers arising from the research and an operational meeting for all those interested in participating in its ongoing collaboration. The continuing medical educationapproved format will again feature a well-balanced mix of didactic instructional lectures, interactive symposia, hands-on product demonstrations and professional interaction. This Annual Scientific Conference is the most important spine meeting in Canada and a must for anyone involved in administering or administrating spine care.

\section{Canadian Paediatric Spine Society Presentations}

Experiences in implementing the EOS radiographic system and SterEOS three-dimensional module. Doug Hill, ${ }^{1,2}$ Edmond Lou, ${ }^{1}$ Sarab Soutbon, ${ }^{1,2}$ Katbleen Shearer. ${ }^{2}$ From the ${ }^{1}$ University of Alberta and ${ }^{2}$ Alberta Health Services.

Background: The American Academy of Orthopaedic Surgeons, Scoliosis Research Society, Pediatric Orthopaedic Society of North America and American Academy of Pediatrics believe that the principles of ALARA (as low as reasonably allowable) should be applied in diagnostic imaging of children to decrease radiation exposure. The EOS radiography system is becoming more common for pediatric spine follow-up primarily due to reduced radiation exposure. SterEOS produces a threedimensional spine model by identifying anatomic landmarks on frontal and lateral radiographs, which conflicts with the ALARA principle. We describe the use of the EOS radiographic system in Edmonton for spine care, and review how the SterEOS 3D package is used for research purposes and the barriers to its broad clinical use. Methods: The EOS radiography system and the SterEOS 3D package were purchased in Edmonton in 2015 and 2016, respectively, with financial support from the Stollery Children's Hospital Foundation. The main selling features were low-dose radiation and three-dimensional spine modelling. The EOS radiography system entirely replaced pediatric spine imaging, with many images taken with the microdose setting. Results: Since EOS' installation, $75 \%$ of imaging continued to be posteroanterior-only, which precluded three-dimensional modelling. Several research studies in Edmonton have used the SterEOS 3D module around designing novel braces, establishing progression models and the gold standard for assessment of the use of ultrasound to monitor scoliosis. Reconstruction time can be reduced to about 30 minutes. Conclusion: The barriers to routine clinical use of the SterEOS 3D module are reluctance to request additional lateral radiograph based on ALARA, and lack of time and funding for clinical personnel to reconstruct three-dimensional images for clinic use. Time to reconstruct the three-dimensional image precludes having information for clinical decision-making available while the patient is in clinic. In addition, use is limited on congenital deformities and postop- erative follow-up owing to altered anatomy. To maintain proficiency, a certain volume needs to be routinely completed. Finally, generally accepted standards on how to interpret and use the three-dimensional parameters are lacking.

Risk factors for surgical site infections following spinal instrumentation in children: a case-control study. Hasan Abdullab, ${ }^{1,2}$ Asbleigh Catberine, ${ }^{1}$ Denisa Urban, ${ }^{1}$ Laura Rivera, ${ }^{1}$ Fabio Ferri-de-Barros, ${ }^{2,3}$ Mary Brindle. ${ }^{1,2,3}$ From ${ }^{1}$ Efficiency, Quality, Innovation \& Safety at the Alberta Children's Hospital; the ${ }^{2}$ University of Calgary Cumming School of Medicine; and the ${ }^{3}$ Alberta Children's Hospital.

Background: Health care-associated infections (HAIs) are a major cause of morbidity and mortality. They also place a substantial burden on health care workers and the health care system. Surgical site infections (SSIs) are the most substantial contributors to overall costs associated with HAIs, and are difficult to manage in the presence of implanted devices, particularly in spine surgery. Identification of modifiable risk factors allow for developing care pathways that may decrease SSIs. We sought to analyze risk factors associated with SSIs. Methods: We performed a retrospective analysis of all patients who underwent spinal instrumentation between 2010 and 2015 in a tertiary children's hospital. The incidence of SSIs and compliance with SSI prevention measures were quantified. Known and hypothetical risk factors for SSI in spine instrumentation were analyzed with a case-control (infections-noninfections) design. Results: Three hundred seventy-five patients were included in this study, of whom 15 (4\%) developed SSIs. The infection rate in idiopathic scoliosis was $1.78 \%$. In neuromuscular scoliosis, the infection rate was $12 \%$. As expected, nonmodifiable risk factors, such diagnosis of neuromuscular disorders $(p=0.0003)$ and American Society of Anesthesiologist scores greater than $2(p=0.007)$ were significantly associated with SSIs. Fluid resuscitation volume $(\mathrm{FV})$ post-surgery was found to be significantly higher $(p=0.03)$ in patients who developed SSIs. Multiple logistic regression analysis showed that FV was the only modifiable independent risk factor associated with SSIs $(p=0.004)$. Compliance with antibiotic prophylaxis timing and dosage guidelines was $55 \%$ and $74.3 \%$, respectively; however, this was not significantly associated with SSIs. Previously identified modifiable risk factors such as length of 
operation, surgical drain use and intrawound vancomycin use were not associated with SSIs. Conclusion: We have found a lower SSI rate in pediatric spine instrumentation in our institution as compared with most reports in the current literature. Fluid resuscitation volume postsurgery was the only modifiable variable associated with SSI.

On-site surgical simulation for teaching pedicle screw instrumentation: a pilot study. Alejandro Peiro-Garcia, ${ }^{1}$ Allison $R$. Teles, ${ }^{2}$ David Parsons, ${ }^{1}$ Fábio Ferri-de-Barros. ${ }^{1}$ From the ${ }^{1}$ Pediatric Spine Program, Department of Surgery, University of Calgary, Calgary, Alta.; ${ }^{2}$ Combined Spine Program, Department of Surgery, University of Calgary, Calgary, Alta.

Background: Spine instrumentation with pedicle screws is taught during different levels of surgical education. Most of the current teaching occurs in vivo. Sequential navigated power drilling (SNPD) is an improvement of the classic technique for spine instrumentation with pedicle screws. Previous research documented a significant reduction in operating time and blood loss in scoliosis surgery in our institution since the systematic implementation of SNPD (Ferri-de-Barros et al., American Academy of Orthopaedic Surgeons 2017; Canadian Spine Society 2018). Surgical simulation is an effective tool for studying and teaching technical skills. We developed a simulation model for studying and teaching pedicle screw instrumentation. We would like to present our pilot data to the Canadian Paediatric Spine Society (CPSS) membership with the intent of fostering national collaboration in this research and educational endeavour. Methods: Five spine fellows performed 4 experiments, each using a specific technique. Each experiment consisted of instrumenting a standard simulator with 10 pedicle screws (T3 to T7). A neurosurgeon, blinded to the technique, directly measured accuracy of pedicle screw instrumentation and graded each pedicle screw according to a valid scale. We compared the timed performances of all subjects using $t$ and the $Z$ tests $(\alpha=1 \%)$ and used regression modelling to study the hypothetical effect of different techniques on accuracy and efficiency of pedicle screw instrumentation. Results: We found differences in performance with different levels of training and different techniques among the five fellows. These results require further analysis, which will be concluded for presentation at the CPSS meeting. Conclusion: The experiments can each be completed in about 30 minutes. Invaluable learning has been reported. Ten residents will be recruited to perform the same experiments, which will be repeated with a pretest, test and retention test model. With these data, we will describe the learning curves of different techniques.

Spinal casting in severe early onset scoliosis. Fennifer Dermott, Reinhard Zeller. From the Hospital for Sick Children, Toronto, Ont.

Background: Spinal casting for early onset scoliosis has recently increased in popularity despite it being a refined conservative treatment option since the beginning of the 20th century. At our institution, the primary aim of spinal casting is to delay the initiation of growth-friendly surgical techniques and thus limit the number of surgeries these children may undergo. We present the technical details of spinal casting and evaluate its use as a treatment strategy to delay the initiation of growth-friendly surgical techniques in children with severe early onset scoliosis. Methods: We performed a retrospective chart and radiographic review of 13 consecutive patients who underwent spinal casting between July 2014 and November 2016. A series of 3 casts were applied, each on for 8 weeks, followed by full-time rigid brace wear. The Cobb angle of the largest curve was measured before the initiation of casting (baseline) and compared with the Cobb angle measurements with the child in their spinal cast, in their brace, and out of their brace at their most recent follow-up. Independent $t$ tests were used to compare curve magnitudes to baseline. Results: Thirteen children (7 females) were reviewed. Of these, 7 were idiopathic, 5 syndromic and 1 neuromuscular. The mean age at initial cast application was 3.5 years (range 1.9-5.6 yr). At baseline, the mean Cobb angle was $66.8^{\circ} \pm 17.2^{\circ}\left(\right.$ range $45^{\circ}-91^{\circ}$ ). This improved to $29.3^{\circ} \pm 13.1^{\circ}(p<0.001)$ in cast and $39^{\circ} \pm 16.6^{\circ}$ in brace $(p<0.001)$. At the most recent follow-up, the mean Cobb angle was $54.4^{\circ} \pm 17.1^{\circ}(p=0.0016)$. At a mean follow-up of 24.9 months (range 13.4-39.4 mo), none of the 13 children showed curve progression beyond baseline. Conclusion: Spinal casting in severe early onset scoliosis should be considered an effective conservative management option to minimize curve progression and ultimately delay the need for operative intervention.

Effect of stabilization exercise on back pain, disability and quality of life in adults with scoliosis: a systematic review. Malik Alanazi, Eric C. Parent, Elizabeth Dennett. From the Faculty of Rehabilitation Medicine, University of Alberta.

Background: Adult scoliosis (AS) is the most common spine deformity in the adult population. Back pain is the main symptom that leads patients to seek medical consultation. Stabilization exercise has been reported to be effective in reducing back pain, but the literature has not been reviewed to examine the effects of such exercises on back pain, disability or quality of life in adults with scoliosis. Our objective was to evaluate the effect of stabilization exercise on back pain, disability and quality of life in adults with scoliosis. Methods: We conducted a systematic search in the following databases from inception to Mar. 9, 2017: Medline (Ovid), CINAHL (EBSCO), Embase (Ovid), SportDiscus (EBSCO) and the Cochrane Central Register of Controlled Trials (CENTRAL). Selection criteria were as follows: randomized controlled trials (RCTs), prospective controlled clinical trials and retrospective controlled studies that compared core stabilization exercise to placebo, no treatment or any other type of treatment. Participants had to be diagnosed with AS and be 18 years of age or older. Studies that included participants with any torso or lower-extremity surgery, any injection in the last 6 months, any comorbidity that could affect the spine, red flag signs or with a history of spine trauma were excluded. Results: We found 908 references, which resulted in 630 articles after duplicates were excluded. After screening the titles and abstracts, only 105 articles were included for full-text screening. A total of 98 full-text articles could be retrieved. Only one article fit the selection criteria and was included in this review. The main reasons for exclusion were study design and patient population. The included study presented a low risk of bias for all criteria except blinding. Authors also did not report if the timing of assessments was similar between groups. Conclusion: This review provided limited evidence that stabilization exercise (one 60-min session/wk for $20 \mathrm{wk}$ of exercises developing motor control of the spine and 
pelvis) is effective for reducing back pain or disability and improving quality of life in adults with scoliosis. However, this review highlights the paucity of literature examining the effect of exercise on back pain in adults with scoliosis and strongly suggests that further experimental research is needed.

\section{Abstract Presentations}

\section{5}

Postoperative radiographic spinopelvic measurements gradually change following thoracolumbar fusions for adult spinal deformity. Michael M.H. Yang, Khaled A. Almansoori, Peter Lewkonia, W. Bradley Facobs. From the University of Calgary, Calgary, Alta.

Backround: Adult spinal deformity is an increasingly common problem encountered by spine surgeons. Restoration of spinopelvic balance has been shown to improve outcomes following surgical intervention. However, radiological measurements may change over time following surgical realignment of a patient's global balance. This study evaluates the degree of change among radiographic spinopelvic parameters within the first postoperative year following adult spinal deformity surgery. Methods: A retrospective observational cohort study was performed by reviewing scoliosis images over a 10 -year period to identify patients undergoing degenerative-scoliosis procedures with a thoracolumbar instrumented fusion of more than 5 spinal levels. Among patients who met inclusion criteria, perioperative scoliosis images were compared between early (6-12 wk) and late postoperative (9-18 mo) periods. Nine radiographic parameters were independently measured by 2 spinal surgeons: sacral slope, pelvic tilt, pelvic incidence, lumbar lordosis, pelvic mismatch, thoracic kyphosis, T1-spinopelvic angle, proximal-junction angle and sagittal vertical axis. Independent $t$ tests with Bonferroni correction were performed; $\alpha$ was set at 0.05 . Results: A total of 876 scoliosis patients were identified, 19 of whom met all inclusion criteria. The mean interval between early and late postoperative radiography was 11.8 months. The following radiographic parameters with respective $p$ values had significantly changed over this period; pelvic tilt $+7.1^{\circ}(p=0.0002)$, thoracic kyphosis $+6.7^{\circ}(p=0.003)$ and proximal junction angle $+6.3^{\circ}(p=0.001)$. No changes were noted among sacral slope, pelvic incidence, lumbar lordosis, pelvic mismatch or T1 spinopelvic inclination measurements. Interrater reliability using $\mathrm{K}$ statistics are pending. Conclusion: Pelvic tilt, thoracic kyphosis and proximal junction angles significantly change within the first postoperative year following lower thoracic to sacrum fusions for degenerative scoliosis. Early postoperative radiograph measurements are subject to change following sagittal balance corrections.

\section{7}

Impact of pelvic parameters on clinical outcomes following short segment lumbar fusion. Shandy Fox, Brad Facobs, Stephan du Plessis, Dan Yavin, Roberta Lee. From the University of Calgary, Calgary, Alta.

Background: The importance of spinopelvic balance to patient-reported clinical outcomes has been previously demon- strated in adult spinal deformity patients. Recent literature suggests that maintenance or restoration of spinopelvic balance may also decrease the rate of adjacent segment degeneration after short segment lumbar fusions. The purpose of this study was to assess whether postoperative spinopelvic parameters in patients who underwent 1 to 4 level lumbar fusions had an effect on patient outcomes and health care utilization for lumbar spine-related complaints. Methods: One hundred fifty patients undergoing 1 to 4 level lumbar spinal fusion between 2010 and 2015 were enrolled in a prospective database. Spinopelvic parameters of lumbar lordosis (LL), pelvic incidence (PI), pelvic tilt (PT) and sacral slope (SS) were recorded from preoperative and postoperative imaging. Patients were labelled "matched" if PI:LL was $10^{\circ}$ or smaller and "mismatched" if PI:LL was more than $10^{\circ}$. Postoperative health care use was recorded. Reoperation rates due to adjacent segment degeneration, pseudarthrosis or device-related complications were compared between groups, as were cross-sectional imaging for recurrent or persistent symptoms, postoperative spinal injections, hospital readmission or assessment for lumbar-related causes, and opioid use. Results: Eighty-five patients were "matched", 47 were "mismatched" and 18 did not have adequate imaging. All patients were followed for a minimum of 2 years from the index surgical date. The mismatched group had a nonsignificant increase in postoperative injections (20\% v. $12.7 \%)$ and cross-sectional imaging (22.2\% v. $15.2 \%)$. Reoperation rate was significantly higher in the mismatched group $(20 \%$ v. $7.6 \%)$. Opioid use at 2 years was similar in the matched and unmatched groups (17.7\% v. $20 \%)$. Conclusion: Maintaining or achieving PI:LL $\leq 10^{\circ}$ may result in fewer patients requiring spine injections, cross-sectional imaging and reoperations within 2 years following short segment lumbar fusion. These data suggest that maintenance of spinopelvic balance may have an important clinical effect on outcome after short segment lumbar fusion.

\section{2}

Hardware failure based on extent of sagittal vertical axis correction in adult spinal deformity surgery. Fanine Hsu, ${ }^{1}$ Evan Frangou, ${ }^{1,2}$ Daniel Warren. ${ }^{1,2}$ From the ${ }^{1}$ Island Medical Program, University of British Columbia, Victoria, BC; ${ }^{2}$ Vancouver Island Neurosurgical Foundation, Victoria, BC.

Background: We aim to study whether the extent of spinal correction, based on pre- and post-operative sagittal vertical axis (SVA), is associated with hardware failure in patients receiving spinal deformity surgery. Methods: We performed a retrospective study to analyze spinal deformity correction of short thoracolumbar (2-6 levels of correction) and long thoracolumbar (7-12 levels of correction) performed by 2 surgeons from 2012 2015 at a tertiary care centre in Victoria, British Columbia. We measured SVA using plain films preoperatively and postoperatively at 3 months to 1 year. Pre- and postoperative SVAs were divided into SVA $<$ 5, SVA 5-10 and SVA $>10$. Postoperative hardware failure was determined by imaging. Hardware failure was divided into major (proximal junctional kyphosis, distal junctional failure or rod fracture) and minor (loose screws). Results: Thirty-three patients were involved: 22 women $(66.6 \%)$ and 11 men $(33.3 \%)$. Twelve patients had major 
hardware failure $(36.4 \%)$ and 8 patients had minor hardware failure (24.2\%). Six patients had preoperative SVA $<5$ (group A), 13 had preoperative SVA 5-10 (group B) and 14 had preoperative SVA $>10$ (group C). In group A, 5 patients had postoperative SVA $<5$, of whom 1 patient had major hardware failure (20\%) and 1 patient had minor hardware failure (20\%); 1 patient had postoperative SVA 5-10 and no hardware failure. In group B, 7 patients had postoperative SVA $<5$, of whom 1 patient had major hardware failure (14.3\%) and 4 patients had minor hardware failure (57.1\%); 6 patients had postoperative SVA 5-10, of whom 6 had major hardware failure $(46.2 \%)$ and 2 had minor hardware failure (33.3\%). In group C, 2 patients had postoperative SVA $<5$, of whom 1 had major hardware failure and no patients had minor hardware failure; 9 patients had postoperative SVA 5-10, 4 of whom had major hardware failure $(44.4 \%)$ and 3 of whom had minor hardware failure (33.3\%); 3 patients had postoperative SVA > 10, 2 with major hardware failure $(66.6 \%)$ and none with minor hardware failure. Conclusion: We show that the highest rate of major hardware failure was in patients with uncorrected SVA (group B, postoperative SVA 5-10 and group C, postoperative SVA > 10). The second highest rate of major hardware failure was when SVA was over corrected (group C, postoperative SVA $<$ ). The lowest rate of major hardware failure per group was when SVA was moderately corrected (group B, postoperative SVA $<5$ and group C, postoperative SVA 5-10). A larger prospective study would be needed for further investigation.

\section{5}

Interbody fusion through impaction grafting in adult spinal deformity. Robert Ravinsky, Colby Oitment, Stephen Lewis, Sofia Magana, Gerrit Stuivenberg. From the Toronto Western Hospital, Division of Orthopaedic Surgery, University of Toronto, Toronto, Ont.

Background: Interbody fusion is typically performed with a cage, providing a stable conduit for fusion between vertebral end plates. In addition to being expensive, cages depend on normal end plate geometry and host bone stock. Impaction grafting is an inexpensive alternative that does not rely on these factors. This paper reviews the radiographic outcomes of impaction grafting. Methods: The demographic, surgical and radiographic data from patients undergoing open posterior lumbar interbody fusion (PLIF) were retrieved from a prospective database of surgical procedures, between Jan. 1, 2012 and Dec. 31, 2014. Disc heights were measured using lateral lumbar radiographs pre- and post-operatively and compared with measurements made with computed tomography (CT) imaging. Imaging was used to assess resorption in the first 6 months after surgery. Resorption and fusion were assessed between 6 and 18 months, and beyond 18 months postoperatively. Results: We identified 103 PLIFs for inclusion in our analysis among 75 patients with a mean age of $60.0( \pm 14.8)$ years. The population was predominantly female $(2: 1)$. Disc height was stable over time in all groups, and there were no differences in disc height between those that fused $(n=42, x=10.8 \pm 2.9 \mathrm{~mm})$ and those that did not $(n=26, x=10.6 \pm 3.9 \mathrm{~mm})(p>0.05)$. Imaging showed immediate total resorption of graft in $10 \%$ of patients, and subtotal resorption in another $10 \%$ within the first 6 months after surgery. Rate of resorption increased over time to a final rate of $56 \%$ of discs showing some degree of resorption. Solid interbody fusion occurred in $72 \%$ of patients, and posterior interfacet fusion occurred in $89 \%$ of patients. Qualitatively, the graft initially appears as a solid mass on radiography and CT, and undergoes resorption, reorganization and remodelling over time, taking on a spongy appearance. Conclusion: Although total fusion rates were comparable with the literature, overall interbody fusion rates were lower than what is reported in the literature. Despite resorption, a high fusion rate was observed, with maintenance of the disc height, suggesting the grafts served a load-sharing function.

\section{9}

Direct nerve root stimulation: a novel approach to neuromonitoring for reduction of high-grade spondylolithesis. Christopher Nielsen, Stephen Lewis, Samuel Stranzas, Laura Holmes, Mabeswara Akula. From the Toronto Western Hospital, Division of Orthopaedic Surgery, University of Toronto, Toronto, Ont.

Background: Traditional methods of neuromonitoring for spinal deformity lacks accuracy and reproducibility in monitoring for high-grade spondylolisthesis. Free-run electromyography (EMG) has not been shown to be accurate in detecting radicular injuries in lumbar degenerative surgeries. This project introduces direct nerve stimulation (DNS) as a novel alternative to nerve root monitoring during reduction of high-grade spondylolisthesis. Methods: Prospectively collected intraoperative neuromonitoring data including motor evoked potential (MEP), somatosensory evoked potential (SSEP) and free-run EMG, were collected from pediatric patients who underwent posterior reductions for high-grade L5/S1 spondylolisthesis. A fourth modality that involved DNS of the L5 and S1 nerve roots was recorded by placing a stimulator directly on the exposed nerve root and recording threshold stimulus. All modalities were recorded before and during reduction, and before closure. Results: Five patients with high-grade spondylolisthesis were included in the analysis, 4 with grade IV and 1 with grade V. All patients had positive bilateral straight leg raises. Four of the patients had intact preoperative motor examinations; 1 patient had unilateral extensor hallucis longus weakness. Three patients did not have any neuromonitoring alerts during their surgical procedure; the mean change in their threshold value from baseline to final stimulation for the L5 nerve root was $1.3 \mathrm{~mA}$. Two patients had intraoperative alerts of MEPs, SSEPs and EMGs, which resulted in greater nerve root stimulation threshold values at closing compared with baseline. One patient showed a 3-fold increase in DNS threshold, whereas a second patient had a 25 - to 30 -fold increase. In both cases, further nerve root exploration and decompression were performed. Both patients had immediate postoperative neurologic deficits in bilateral ankle dorsiflexion. Conclusion: After establishing prereduction thresholds for DNS, increases in DNS threshold were associated with postoperative neurologic deficits in cases of posterior reduction of high-grade spondylolisthesis. Direct nerve stimulation provided an accurate measure of nerve function in this series. Recognizing increases in DNS thresholds intraoperatively can alert the surgeon to potential real-time nerve injuries. A larger multicentre series can help determine the utility of this technique. 


\section{6}

Minimal clinically important difference for CPCHILD Questionnaire following scoliosis surgery in cerebral palsy. Firoz Miyanji, ${ }^{1,2}$ Luigi Nasto, ${ }^{1}$ Paul Sponseller, ${ }^{3}$ Tracey Bastrom, ${ }^{4}$ Suken Shab, ${ }^{5}$ Amer Samdani, ${ }^{6}$ Michelle Marks, ${ }^{7}$ Burt Yaszay, ${ }^{4}$ Unni Narayanan, ${ }^{8}$ Peter Newton. ${ }^{4}$ From the ${ }^{1} \mathrm{BC}$ Children's Hospital, Vancouver, BC; ${ }^{2}$ University of British Columbia, Vancouver, BC; ${ }^{3}$ Johns Hopkins University, Baltimore, Md.; 'Rady Children's Hospital, San Diego, Calif.; ${ }^{5}$ Nemours/Alfred I. duPont Hospital for Children, Wilmington, Del.; 'Shriners Hospital for Children, Philadelphia, Pa.; ${ }^{7}$ Setting Scoliosis Straight, San Diego, Calif.; ${ }^{8}$ The Hospital for Sick Children, Toronto, Ont.

Background: Patient-reported outcomes have evolved to include an understanding of the patient's perspective of change. Minimal clinically important difference (MCID) is defined as the minimum amount of change important to the patient or caregiver. The CPCHILD Questionnaire is designed to measure health-related quality of life in patients with cerebral palsy (CP) and has been used to study outcomes of scoliosis surgery in this population. However, the MCID threshold for this questionnaire following scoliosis surgery remains unknown. Our aim was to define an MCID threshold score for the questionnaire for patients undergoing scoliosis surgery with a minimum 2-year follow-up. Methods: An anchor-based method using a prospective, multicentre CP scoliosis surgical database was conducted. All consecutive CP patients with 2 or more years of follow-up who underwent deformity correction were included. CPCHILD scores were collected at baseline and 2 years after surgery. Caregivers were asked (anchor questions) to rate on a 5-level Likert-type scale the effect of surgery with regard to improvement in quality of life (QOL), comfort (COM), ease of care (EOC), and general health $(\mathrm{GH})$ of their child at 2 years' follow-up. The change in CPCHILD total score (TS) was then compared with the patient or caregiver responses to the anchor questions. Linear and logistic regression models were used to calculate the MCID. Results: One hundred thirty-two patients were included. The MCIDs for significant improvements in QOL, COM and EOC were $10(p=0.016), 15.8(p=001)$, and $6.8(p=0.022)$ points, respectively. The GH anchor did not reach statistical significance. In patients who reached the MCID, the odds of improvement with surgery was 1.77 -fold higher in QOL, 4.9-fold higher in COM, and 1.48-fold higher in EOC than in those that did not. The probability of patients improving after surgery if the MCID was reached was $63.9 \%$, $83 \%$ and $59.5 \%$ in anchors QOL, COM and EOC, respectively. Conclusion: No univocal estimate of MCID value for CPCHILD TS can be given. However, using an anchor-based method, we provide MCID values in the CPCHILD TS to predict a significant improvement in QOL, COM and EOC 2 years after scoliosis surgery.

95

A prospective study of the improvement in health-related quality of life following surgical treatment of lumbosacral spondylolisthesis in young patients. Étienne BourassaMoreau, ${ }^{1,2,3}$ Jean-Marc Mac-Thiong, ${ }^{1,3,4}$ Timotby Hresko, ${ }^{5}$ Stefan Parent, ${ }^{1,3,4}$ Dan 7. Sucato, ${ }^{6}$ Lawrence G. Lenke, ${ }^{7}$ Michelle Marks, ${ }^{8}$ Hubert Labelle. ${ }^{1,4}$ From the ${ }^{1}$ Université de
Montréal, Montreal, Que.; ${ }^{2}$ Oxford Spinal Unit, Oxford, United Kingdom; ${ }^{3}$ Hôpital du Sacré-Coeur, Montreal, Que.; ${ }^{4}$ CHU Sainte-Justine, Montreal, Que.; ${ }^{5}$ Boston Children's Hospital, Boston, Mass.; ${ }^{6}$ Texas Scottish Rite Hospital, Dallas, Tex.; ${ }^{7}$ The Spine Hospital New York Presbyterian, New York, NY; ${ }^{8}$ Setting Straight Scoliosis Foundation, San Diego, Calif.

Background: Large prospective studies are needed to clearly define the benefits of surgery in the young patient population. The objective of this study was to assess the health-related quality of life (HRQOL) changes after surgical treatment of pediatric lumbosacral spondylolisthesis. Methods: A prospective multicentre study of surgical lumbosacral spondylolisthesis patients, younger than 25 years with a minimum of 2 years follow-up, was performed. The main outcome was Scoliosis Research Society (SRS-22) questionnaire scores compared preoperatively and at 2 years' postoperative follow-up. Results: Eighteen low-grade and 64 high-grade slips patients met our inclusion criteria. Patient HRQOL was significantly improved 2 years after surgery for all domains and for the total score of the SRS-22 questionnaire. In 69 patients $(84 \%)$, SRS total score improved, and 54 patients (66\%) improved their SRS-22 score by a clinically significant difference. All subscore domains of the SRS-22 questionnaire significantly improved in high-grade slips; however, only pain, function and total subscores improved for low-grade slips. Conclusion: This is the largest study comparing the HRQOL before and after surgery in young patients with low- and high-grade lumbosacral spondylolisthesis. The results show that HRQOL was significantly improved by surgery, especially for high-grade patients. This study helps clinicians to better counsel patients with regard to the benefits of surgery for lumbosacral spondylolisthesis.

\section{5}

Measuring the Cobb angle in scoliosis patients: a comparison of manual and computer-generated measurements. Nick Pasic, Parham Rasoulinejad, Chris Bailey, Fabad Al Helal, Shuo Li, Laura Churchill. From Western University, London, Ont.

Background: Our objectives were to assess the interobserver reliability of Cobb angle measurements generated manually by 2 independent expert observers and by an automated system. Methods: We performed a retrospective case review to identify patients diagnosed with scoliosis who had appropriate anteroposterior (AP) radiographs between 2002 and 2017 at London Health Sciences Centre. Two expert independent assessors performed a blinded radiographic review of 99 patients. Manual Cobb angle measurements were done on digitized AP images using picture archiving and communication system (PACS). Cobb angles were measured by identifying the end vertebrae of the curve deformity and drawing intersecting lines on the superior and inferior end plates of these end vertebrae. Computergenerated measurements were completed on 40 patients using the Multi-View Correlation Network (MVC-Net) system. Interrater agreement was estimated using the intraclass correlation coefficient (ICC). Results: The mean difference between expert assessors was $3.84^{\circ}$ (standard deviation [SD] $4.58^{\circ}$ ), with an ICC of $0.93(p<0.01,95 \%$ confidence interval [CI] 0.89 to 0.98 ). The mean difference between expert 1 and the MVC-Net 
system was $27.68^{\circ}\left(\mathrm{SD} 17.61^{\circ}\right)$, with an ICC of $0.03(p=0.30$, $95 \% \mathrm{CI}-0.01$ to 0.22 ). The mean difference between expert 2 and the MVC-Net system was $27.34^{\circ}$ (SD 19.36 $)$, with an ICC of 0.06 ( $p=0.13,95 \% \mathrm{CI}-0.07$ to 0.22$)$. Conclusion: Similar to previous studies, interrater reliability between expert observers was excellent, supporting the use of the manual Cobb angle measurement technique. Although a computerized method of measurement would add efficiency to clinical decision-making, the MVC-Net system proved unreliable compared with expert raters and does not currently offer clinical utility. Factors such as variable patient anatomy and poor-quality radiographic films are easily overcome by human raters but present unique challenges for the MVC-Net system. Training of the system is ongoing and will need to reconcile these issues before it can be used effectively in the assessment of scoliosis patients.

\section{2}

Nanodose protocol for Cobb angle assessment in early onset scoliosis: preliminary results of a phantom-based and clinically validated study. Peter $H$. Pedersen, ${ }^{1}$ Fred Xavier, ${ }^{2}$ Claudio Vergari, ${ }^{3}$ Alexia Tran, ${ }^{4}$ Antoine Faeger, ${ }^{4}$ Pierre Laboudie, ${ }^{4}$ Wafa Skali, ${ }^{3}$ Soren P. Eiskjaer, ${ }^{1}$ Raphael Vialle. ${ }^{4}$ From the ${ }^{1}$ Aalborg University Hospital, Denmark; ${ }^{2}$ Department of Surgery (Spine), Dalhousie University, Halifax, NS; ${ }^{3}$ École nationale supérieure d'arts et métiers, Paris, France; ${ }^{4}$ Chirurgie Ortopedique Pediatrique, Hopital Armand Trousseau, Paris, France.

Background: Scoliosis is a three-dimensional deformity of the spine characterized by three-dimensional clinical parameters. Nevertheless, the two-dimensional Cobb angle remains an important and widely used radiologic measure in clinical practice. Repeated imaging is required for the assessment and follow-up of scoliosis patients. Then, high doses of absorbed radiation increase the risk of developing radiation-induced cancer in such patients. Microdose radiographic imaging is already available in clinical practice, but the radiation delivered to the patient can be further reduced. To address this issue, we aimed to validate a new nanodose full-spine protocol for reproducible Cobb angle assessment. Methods: An anthropomorphic child phantom was used to establish a lowest possible dose in the EOS imaging system, still allowing Cobb angle measurements, defined as the "nanodose". A pilot group of 17 children less than 12 years of age was assessed with microdose and additional nanodose full-spine imaging modalities. Two patients had to be excluded owing to metal implants and subsequent image artifacts. Intra- and inter-observer reliability of determining reliability of Cobb angle measurements was performed according to the ISO 5725 standard. Results: Microdose full-spine imaging settings were $60 \mathrm{kV}$ and $80 \mathrm{~mA}$ for frontal view radiographs, corresponding to $30 \mathrm{mGy} \cdot \mathrm{cm}^{2}$. Nanodose settings were $60 \mathrm{kV}$ and $20 \mathrm{~mA}$ for frontal view radiographs, corresponding to $6.8 \mathrm{mGy} \cdot \mathrm{cm}^{2}$ exposure. These preliminary in vivo data showed good reliability and repeatability between microdose imaging and the new protocol. There was a 6 -fold reduction in radiation exposure from microdose to nanodose full-spine imaging. Pooled results for 4 observers indicated inter- and intraobserver variability of less than $5^{\circ}$ using $95 \%$ confidence intervals, which is within general accepted standards. Conclusion: We propose a new nanodose protocol for routine full-spine imaging of children being followed-up for scoliosis before surgery. Although image quality was, as expected, worse than with standard dose and microdose, our study showed good repeatability for Cobb angle measurements with the new nanodose measurements. Exposure from the nanodose protocol is $1 / 6$ that of microdose, equivalent to about 1 day of natural background exposure.

\section{1}

Sagittal balance and spinopelvic parameters in patients with adolescent idiopathic scoliosis managed with intraoperative skull-femoral traction. Alejandro Peiro-Garcia, ${ }^{1,2}$ Garielle Brown, ${ }^{2}$ Madalene Earp, ${ }^{2}$ David Parsons, ${ }^{1,2}$ Fabio Ferri-de-Barros. ${ }^{1,2}$ From the ${ }^{1}$ Alberta Children's Hospital, Calgary, Alta.; ${ }^{2}$ University of Calgary, Calgary, Alta.

Background: Previous studies have raised concern about excessive correction of lumbar lordosis in adolescent idiopathic scoliosis (AIS) patients managed with intraoperative traction. Our purpose is to compare postoperative sagittal balance and spinopelvic parameters in 2 cohorts of AIS patients who underwent surgery with or without intraoperative skull-femoral traction (IOSFT). Methods: The medical and radiologic records of 113 AIS patients managed by posterior spinal instrumented fusion were reviewed. Eighty-five patients underwent surgery with IOSFT (traction group) and 28 patients underwent surgery without traction (nontraction group). The radiologic outcomes and complication rates were compared between the 2 groups before and 2 years after surgery. A $p$ value of less than 0.05 was considered significant. Results: No significant differences were found in sagittal balance and pelvic parameters between both groups at 2 years' follow-up. Median coronal plane correction was significantly greater in the traction group, although the patients in the traction group had smaller preoperative Cobb angles and implant density. No traction-related complications were reported. Conclusion: According to our results, the use of IOSFT in posterior arthrodesis for managing AIS did not negatively impact sagittal balance or pelvic parameters. The use of IOSFT combined with adequate spinal cord monitoring is safe. Further research is required to investigate the generalizability of our findings.

\section{6}

Quality, safety and value of innovation in scoliosis surgery: intraoperative skull-femoral traction and navigated sequential drilling. Fonatban Bourget-Murray, Alejandro PeiroGarcia, Garielle Brown, Madalene Earp, David Parsons, Fabio Ferri-de-Barros. From the Cumming School of Medicine, University of Calgary, and the Department of Surgery, Division of Pediatric Orthopedic Surgery, Alberta Children's Hospital, Calgary, Alta.

Background: Posterior spinal instrumentation and fusion (PSIF) is a common and expensive surgery for the management of adolescent idiopathic scoliosis (AIS). Since 2010, our institution has implemented 2 surgical strategies to mitigate the growing costs associated with this surgery: intraoperative skull-femoral traction (IOSFT) and navigated sequential drilling (NSD). This study compares the clinical and financial 
impact for 2 different surgical strategies used for pedicle screw hole preparation: NSD and a navigated awl technique, both used with IOSFT. Methods: We reviewed the medical records of 125 consecutive patients who underwent a singlestage PSIF for the treatment of AIS between 2008 and 2015 at a single institution. We identified 3 cohorts based on the different surgical strategies used in the treatment of AIS: traditional techniques $(n=28)$, IOSFT $(n=45)$, and IOSFT plus NSD $(n=52)$. Primary outcomes focused on reportable quality indicators: operative time, prevalence of cases requiring extended operating room time, need for blood transfusion, length of hospital stay and total cost per surgery. Secondary outcomes included implant density, degree of spine deformity correction and complication rate. Results: All primary outcomes improved significantly in the IOSFT and NSD cohort $(p<0.001)$. Mean operating time decreased by 59\%, ranging from 462 to 185 minutes. Use of late operating room hours fell from $89 \%$ to $0 \%$, transfusion rate from $64 \%$ to $1.9 \%$ and length of hospital stay from 5 to 4 days. Comprehensive cost per case decreased by $24 \%$. These savings were mostly accounted for by the reduced length of hospital stay and operating time. Conclusion: The implementation of IOSFT and NSD delivered superior quality, safety and value-of-care relative to the conventional awl technique. These surgical strategies were performed without increased risk of perioperative complications, while allowing us to reduce costs by $16 \%$ per case. The data presented may have considerable implications in health resource use for scoliosis surgery. Prospective larger scale research is warranted to investigate the generalizability of our findings.

\section{4}

Anterior vertebral body tethering for the treatment of idiopathic scoliosis: feasibility, outcomes and complications. Firoz Miyanji, ${ }^{1}$ Luigi Nasto, ${ }^{2}$ Eva Habib, ${ }^{2}$ Andrea Simmonds. ${ }^{2}$ From the ${ }^{1}$ BC Children's Hospital and University of British Columbia, Vancouver, BC; ${ }^{2}$ BC Children's Hospital, Vancouver, BC.

Background: Anterior vertebral body tethering (AVBT) has recently sparked interest as a possible alternative in the management of progressive idiopathic scoliosis (IS). To date, limited available data exist regarding the efficacy and complication rate with AVBT. Our aim was to evaluate the clinical, radiographic and perioperative outcomes and complication rates to determine the efficacy of AVBT in skeletally immature patients with IS. Methods: A retrospective review of all consecutive patients treated with AVBT between 2012 and 2016 was conducted after Institutional Review Board approval. Demographic data were collected from chart review. Preoperative and most recent follow-up radiographic parameters were measured by an independent reviewer. Perioperative outcome variables and complication data were obtained from chart review. Clinical success was set a priori as major coronal Cobb angle $\leq 30^{\circ}$ at most recent follow-up. Results: Thirty-two patients with 34 procedures were included in the analysis. Mean age at surgery was $13.6 \pm 1.4$ years, and patients were mostly female (93.8\%). Mean Risser grade was $0.77 \pm 0.79$ with a mean follow-up of $17.8 \pm 10.9$ months. Mean major preoperative Cobb angle was $50.6^{\circ} \pm 8.6^{\circ}$, improved to mean of $18.3^{\circ} \pm 9.5^{\circ}$ at most recent follow-up (\% correction: $64.3 \%, p<0.001$ ). Significant spontaneous curve correction was also observed in the uninstrumented curves $(48.7 \% \pm 24.2 \%, p<0.001)$. Thoracic axial rotation significantly improved from $15.0^{\circ} \pm 4.2^{\circ}$ to $8.0^{\circ} \pm 4.1^{\circ}(p<$ $0.001)$ as measured by scoliometer. The mean number of instrumented levels was $6.8 \pm 0.9$, with a mean operating room time of $348.4 \pm 84 \mathrm{~min}$. Mean estimated blood loss was $252.8 \pm 83.4 \mathrm{~cm}^{3}$, with no patient requiring allogeneic blood. Mean length of hospital stay was $5.3 \pm 1.0$ days, with $84.3 \%$ of patients returning to full activity at 3 months. Clinical success was noted in $96.6 \%$ of patients at most recent followup. We noted a $23.5 \%$ complication rate; however, there were no readmissions to hospital or reoperations in this cohort. Conclusion: Anterior vertebral body tethering is an effective technique in obtaining clinical success in skeletally immature patients with IS. No major complication was noted, suggesting an acceptable safety profile. Early results appear promising; however, longer-term follow-up is needed to determine true clinical benefits.

26

Short-term complications with vertical expandable prosthetic titanium rib and magnetically controlled growing rods to manage early onset scoliosis. Alejandro Peiro-Garcia, ${ }^{1,2}$ Fonathan Bourget-Murray, ${ }^{2}$ Fabio Ferri-de-Barros, ${ }^{1,2}$ David Parsons. ${ }^{1,2}$ From the ${ }^{1}$ Alberta Children's Hospital, Calgary, Alta.; ${ }^{2}$ University of Calgary, Calgary, Alta.

Background: Early onset scoliosis (EOS) is a challenging problem for spine surgeons and has been managed with different growth-friendly instrumentation systems. Although rib-based devices encourage spinal growth via regular lengthening, the high rate of complications and reoperations leads us to use spine-based devices, such as magnetically controlled growing rods (MCGRs) to mitigate this concern. The objective of this study is to report our short-term complication rates for patients with EOS managed with vertical expandable prosthetic rib (VEPTR) and MCGR. Methods: Thirtyfive EOS patients were included in the study. Twenty patients were included in the VEPTR group and 15 patients were included in the MCGR group. Demographic data and 1 -year postoperative complications and reoperations were reviewed retrospectively. As secondary outcomes, radiographic outcomes were reported preoperatively and 1 year after surgery. Indications for this technique and complications were collected from the chart. Results: Significant differences were found in the complications rate at 1-year, with $65 \%$ complications in the VEPTR group and $6.7 \%$ complications in the MCGR group $(p<0.001)$. The reoperation rate at 1 -year was also significantly higher in the VEPTR group (50\% v. $6.7 \%$ in the MCGR group, $p=0.006)$. Eleven patients in the MCGR group received a staged MCGR insertion. Conclusion: According to our results, VEPTR has been shown to have a significantly higher complication and reoperation rate at 1 -year postsurgery compared with MCGR. Staged MCGR insertion may explain lower implant failure in the MCGR group. To our knowledge, this is the first series of EOS patients that compares short-term complications and reoperation rates in VEPTR and MCGR. 


\section{0}

Early spinal size gain and alignment changes in children with early onset scoliosis who receive MAGEC rods: a preliminary study using the EOS imaging system. Fred Xavier, ${ }^{1}$ Antoine Faeger, ${ }^{2}$ Raphael Vialle. ${ }^{2}$ From the ${ }^{1}$ Department of Surgery (Spine), Dalhousie University, Halifax, NS; ${ }^{2}$ Chirurgie Orthopedique Pediatrique, Hopital Armand Trousseau, Paris, France.

Background: Avoiding the definitive burden of spinal fusion surgeries, growing rod techniques have been used for decades as a means of growth management in severe cases of early onset scoliosis. More recently, magnetically controlled rods were introduced as an alternative to the traditional forms of growing rods. Spinal lengthening for this newer technique is performed magnetically and noninvasively during outpatient visits. In this pilot study, we assessed the early signs of improvement before distraction in the patients who received the MAGEC growing rod system (Ellipse Technologies Inc.). Methods: Nine children ( 2 boys and 7 girls) with early onset scoliosis were treated with magnetically controlled growing rods. Full-spine radiographs, using the EOS imaging system, were taken preoperatively and at the first follow-up visit before lengthening. We measured spinal length from T1 to L5, frontal Cobb angle, kyphosis and lordosis. We also assessed the early impacts of frontal and sagittal alignment changes in the overall health of the patients. Results: The mean age of our group was 10.8 (range 8-15) years. Our mean postoperative follow-up control time postsurgery was at 4.4 (range 2-9) months. The T1-L5 length increased by mean 3.1 (range $0.7-6.3$ ) $\mathrm{cm}$. There was a reduction in the magnitude of the main frontal curve by an average of $17^{\circ}$ (range $0.8^{\circ}-39^{\circ}$ ). Conclusion: Severe forms of early onset scoliosis in children carry a great risk of rapid spinal deformity progression. Adding to the cosmetic changes, pulmonary insufficiency could lead to significant respiratory failure and a reduced life expectancy in extreme cases. The MAGEC growing rod system provides safe and rapid results. Our preliminary data confirmed the swift changes in spinal size gain and alignment with the implantation of the rods, before the activation of the magnetic system. There was an overall improvement in the quality of life of our patients within the first few months after surgery.

\section{8}

Postoperative distal coronal decompensation after fusion to L3 for idiopathic scoliosis is affected by sagittal pelvic parameters. Stephen Lewis, Kato So, Reinhard Zeller, Sofia Magana. From the Hospital for Sick Children, Division of Orthopaedic Surgery, University of Toronto, Toronto, Ont.

Background: Sparing the distal fusion levels in adolescent idiopathic scoliosis (AIS) surgery is beneficial for better postoperative mobility, but late distal decompensation is of concern. The L3 can be chosen as the lower instrumented vertebra (LIV) in posterior fusion, but progressive angulation of the L3/4 disc is commonly experienced. Elucidating the risk factors for late decompensation in immediate postoperative radiographs will help us understand the optimal corrections for long-term outcomes. Methods: We retrospectively analyzed 54 consecutive AIS patients who underwent posterior fusion to L3 from 2007 to 2012. Patients' demographic data and imme- diate and 2-year postoperative standing whole spine radiographs were investigated. Late decompensation was defined as progressive increase of $\mathrm{L} 3 / 4$ disc wedging angle at 2 years. Coronal, sagittal and rotational radiographic parameters were compared between patients with and without decompensation. Patient Scoliosis Research Society (SRS-30) questionnaire scores were collected as a patient-reported outcome. Results: The mean age of patients was 14.4 years, and mean fusion level was 12 (range 6-15). Twenty-six patients (48\%) experienced progressive L3/4 disc wedging, with 7 patients showing more than $5^{\circ}$ increase. Among the parameters compared, patients with decompensation showed significantly larger pelvic tilt (PT; $14.2^{\circ}$ v. $\left.6.1^{\circ}, p=0.007\right)$ and smaller sacral slope (SS; $34.1^{\circ}$ v. $\left.39.7^{\circ}, p=0.02\right)$ on the immediate postoperative radiograph. Rotation of L3 (1.2 v. 0.8 on Nash-Moe grade, $p=0.07$ ) and $\mathrm{T} 1$ pelvic angle tended to be different $\left(8.9^{\circ}\right.$ v. $\left.4.6^{\circ}, p=0.09\right)$. Multivariate analysis revealed that larger L3 rotation, smaller SS and larger PT are significant risk factors for decompensation. Patient SRS-30 scores did not differ significantly between the 2 groups ( 4.0 v. $4.1, p=0.44$ ). Conclusion: Pelvic retroversion at the immediate postoperative status was found to be the risk factor for late distal coronal decompensation after fusion to L3 for AIS. Proper sagittal alignment correction is imperative to ensure long-term outcomes.

92

Establishing guidelines for surgical reduction in high-grade L5-S1 spondylolisthesis based on quality of life measures. Fean-Marc Mac-Thiong, ${ }^{1,2,3}$ Étienne Bourassa-Moreau, ${ }^{1,3,4}$ Timotby Hresko, ${ }^{5}$ Stefan Parent, ${ }^{1,2,3}$ Dan Sucato, ${ }^{6}$ Lawrence Lenke, ${ }^{7}$ Michelle Marks, ${ }^{8}$ Hubert Labelle. ${ }^{1,2}$ From the ${ }^{1}$ Université de Montréal, Montreal, Que.; ${ }^{2}$ Centre hospitalier universitaire Sainte-Justine, Montreal, Que.; ${ }^{3}$ Hôpital du SacréCoeur, Montreal, Que.; ${ }^{4}$ Oxford Spinal Unit, Oxford, United Kingdom; ${ }^{5}$ Boston Children's Hospital, Boston, Mass.; ${ }^{6}$ Scottish Rite Hospital, Dallas, Tex.; ${ }^{7}$ The Spine Hospital New York Presbiterian, New York, NY; ${ }^{8}$ Setting Straight Scoliosis Foundation, San Diego, Calif.

Background: Surgical reduction of slip grade and lumbosacral kyphosis in high-grade isthmic spondylolisthesis remains unclear. In particular, previous studies have not analyzed the influence of surgical reduction on the quality of life. This study aims to analyze the relation between surgical reduction and improvement in quality of life in surgical patients with high-grade spondylolisthesis. Methods: A prospective cohort of 51 patients $(14.9 \pm 3.14 \mathrm{yr})$ with high-grade lumbosacral spondylolisthesis was followed for a minimum of 2 years after surgery. Scoliosis Research Society (SRS-22) scores, slip grade, lumbosacral angle, pelvic incidence, pelvic tilt and sacral slope were assessed before surgery and at latest follow-up. Statistical analyses were performed for the complete cohort, and after subdividing the patients based on their preoperative pelvic balance (balanced v. unbalanced), using nonparametric tests and a level of significance of 0.05. Results: Postoperative SRS-22 scores and the improvement in total SRS-22 score were similar between patients with a postoperative slip grade of 1, 2 or $>2$. Postoperative SRS-22 scores and the improvement in total SRS-22 score were not directly associated with the postoperative value or the improvement in lumbosacral angle. However, a low postoperative pelvic tilt was correlated 
with increased satisfaction, better function and greater improvement in total SRS score, especially for patients with a preoperative unbalanced pelvis. Postoperative pelvic tilt was mainly associated with postoperative lumbosacral angle. Conclusion: Surgical reduction of slip grade or lumbosacral angle in young patients with high-grade spondylolisthesis is not directly associated with an improvement in quality of life. However, postoperative improvement in quality of life is strongly associated low pelvic tilt, which in turn is strongly correlated with an improved lumbosacral angle. Therefore, improving the lumbosacral angle during the surgery is recommended in selected patients to decrease pelvic tilt and optimize quality of life.

22

Staged MAGEC rods insertion to manage early onset scoliosis. Alejandro Peiro-Garcia, ${ }^{1,2}$ Jonatban Bourget-Murray, ${ }^{2}$ David Parsons, ${ }^{1,2}$ Fabio Ferri-de-Barros. ${ }^{1,2}$ From the ${ }^{1}$ Alberta Children's Hospital, Calgary, Alta.; ${ }^{2}$ University of Calgary, Calgary, Alta.

Background: Magnetically controlled growing rods (MCGRs) are currently the gold standard of treatment for early onset scoliosis (EOS). The high rate of reoperation secondary to hardware failures leads us to develop new strategies to mitigate this concern. In 2014, we introduced a staged surgery for MCGR consisting of anchor insertion in the first stage, followed by a halo-gravity period and insertion of MCGRs in the second stage. The objective of this study is to report our radiographic outcomes and complication rates for patients with EOS managed with this technique. Methods: Seventeen EOS patients were managed in 2 stages. During the first stage, anchor points and halo-gravity were applied, followed by halo-gravity traction. At a second stage, halogravity was removed and MCGRs were inserted. Demographic and radiographic outcomes, and complications, were reviewed retrospectively with a median follow-up of 2 years. Radiographic outcomes were reported preoperatively and after the second stage. Indications for this technique and complications were collected from the chart. Results: Indications for 2-stage surgery were short trunk height, stiff curves, severe kyphosis, poor bone density, dislodgement of proximal anchor points in previous constructs and severe curves. The median percentage of main curve Cobb angle correction was $48.7 \%(37 \%-57 \%)$, the median T1T12 increase was $27(17-29) \mathrm{mm}$, and median T1-S1 increase was $39(30-50) \mathrm{mm}$. The complication rate was $5.88 \%$. No infections were reported. No neurologic complications or anchor dislodgement were reported. Conclusion: According to our results, the staged MCGR insertion combined with halo-gravity traction has been shown to improve Cobb angles and increase T1-T12 and T1-S1 heights with a low complication rate. To our knowledge, this is the first series of EOS patients reporting the staged insertion of MCGR combined with halo traction.

29

Assessing health-related quality of life in cerebral palsy following scoliosis surgery: Is the CPCHILD measuring what we want? Firoz Miyanji, ${ }^{1,2}$ Luigi Nasto, ${ }^{1}$ Tracey Bastrom, ${ }^{3}$ Paul Sponseller, ${ }^{4}$ Suken Shab, ${ }^{5}$ Amer Samdani, ${ }^{6}$ Michelle Marks, ${ }^{7}$ Unni Narayanan, ${ }^{8}$ Peter O. Newton. ${ }^{3}$ From the ${ }^{1} \mathrm{BC}$ Children's Hospital, Vancouver, BC; ${ }^{2}$ University of British Columbia, Vancouver, BC; ${ }^{3}$ Rady Children's Hospital, San
Diego, Calif.; ${ }^{4}$ Johns Hopkins University, Baltimore, Md.; ${ }^{5}$ Nemours/Alfred I. duPont Hospital for Children, Wilmington, Del.; ${ }^{6}$ Shriners Hospital for Children, Philadelphia, Pa.; ${ }^{7}$ Setting Scoliosis Straight, San Diego, Calif.; ${ }^{8}$ The Hospital for Sick Children, Toronto, Ont.

Background: The CPCHILD questionnaire is a reliable and validated condition-specific outcome measure of health-related quality of life (HRQOL) for children with severe cerebral palsy (CP). However, the CPCHILD's responsiveness with respect to scoliosis surgery remains unreported. Recently, it has been used to assess outcomes of spinal fusion in this population with conflicting results. Our aim was to assess the responsiveness (sensitivity to change) of the CPCHILD questionnaire after scoliosis surgery. Methods: Primary caregivers of consecutive CP patients with 2 or more years' follow-up after scoliosis surgery completed the CPCHILD at baseline, 1 and 2 years after surgery. Concurrently, caregivers were asked to rate on a 5-level ordinal scale their perception of the effects of surgery (from deteriorated a lot to improved a lot) with respect to 4 items: ease of care (EOC), comfort (COM), general health $(\mathrm{GH})$ and overall quality of life (QOL), at 1 and 2 years' follow-up. Effect size (ES) and standardized response mean (SRM) for the CPCHILD total and domain scores were calculated. Spearman correlation coefficients were calculated between change in CPCHILD score after surgery and caregivers' responses to the 4 questions. Results: We included 173 patients with a mean age of $14( \pm 2.6)$ years, most of whom with a Gross Motor Function Classification System (GMFCS) level 5 (81\%). Most patients rated effect of surgery as significantly "improved" in all anchors compared with "no change" or "deteriorated" $(p<$ 0.001). At 2 years' follow-up, CPCHILD total scores significantly improved $(p<0.001)$, as did scores for all expected domains (personal care and activities of daily living $[p=0.002]$; positioning and mobility $[p<0.001]$; COM $[p=0.005]$; GH $[p=0.004]$; and overall QOL $[p<0.001])$, but not for communication $(p=0.145)$. A moderate $(0.50 \leq$ effect size $[\mathrm{ES}]<0.80)$ responsiveness was observed at 2 years' follow-up across the anchor responses. Strongest correlation at 2 years' follow-up was noted between total score and anchors QOL, COM and EOC $(r=0.33,0.39$ and 0.31 respectively); QOL domain and anchor COM $(r=0.33)$; personal care and COM domains and EOC ( $r=0.3$ and 0.34 , respectively). The weakest correlation was between CPCHILD scores in all domains and GH anchor $(r<0.2)$. Conclusion: A high level of satisfaction was noted among caregivers after scoliosis surgery at 2 years' follow-up in CP patients as demonstrated with significantly improved scores in CPCHILD and meaningful external anchors. The CPCHILD questionnaire demonstrated responsiveness in the domains of QOL, personal care and activities of daily living, COM and total scores at 1 and 2 years' follow-up.

160

Spine surgery infection: Who is at risk? Reina Yao, Hanbing Zhou, Aresh Sepebri, Brian Kwon, John Street. From the Division of Spine, Department of Orthopaedics, University of British Columbia, Vancouver, BC.

Background: Surgical site infections (SSIs) remain a common problem among surgically treated patients with associated increased rates of morbidity and mortality, hospital length of stay and cost. The rate of SSIs reported can range from $0.2 \%$ to 
$16.7 \%$, depending on the type of procedure. Numerous studies have attempted to identify the specific risk factors associated with SSIs but are often limited to a specific diagnosis or procedure. The purpose of this study is to perform a systematic review of SSI risk factors and, more specifically, categorize them into patient-, surgical- and disease-related factors. Methods: We performed a systematic review of published literature on SSI risk factors in adult spine surgery. We included studies that reported risk factors for SSI in adult spinal surgery. We excluded studies involving pediatric patient populations, systematic reviews and metaanalyses. We identified 71 cohort studies, 1 controlled-cohort study, 1 matched-cohort study, 1 matched-paired-cohort study, 12 case-controlled studies, 6 case series and 1 cross-sectional study. Results: We identified the following patient-associated risk factors: diabetes mellitus (type 2), obesity (body mass index $>35$ ), age, subcutaneous fat thickness, chronic steroid use, American Society of Anesthesiologists class > 3, immunosuppression, chronic renal disease, Parkinson disease, current smoker and socioeconomic status (level III). We identified the following disease-associated risk factors: traumatic injury, deformities, tumours (no difference in primary v. metastatic), preoperative radiation and revision surgery (level III). We identified the following surgical factors: prolonged operating room time (level II), stay in an intensive care unit, delay in surgery (trauma), combined anterior-posterior approach, open surgery (v. minimally invasive surgery), surgical invasiveness or levels of instrumentation, lumbosacral junction, number of surgeons and less surgeon experience (level III). Furthermore, early tracheostomy, sex and use of nonsteroidal anti-inflammatory drugs (level III) were not associated with SSI. Conclusion: Surgical site infections are associated with many risk factors that can be patient- or surgery-related. Our review was able to identify important modifiable and nonmodifiable risk factors that can be essential in surgical planning and discussion with patients.

\section{7}

Reduction in surgical site infections with suprafascial intrawound application of vancomycin powder in instrumented posterior spinal fusion: a retrospective case-control study. Ralph T. Schär, ${ }^{1}$ Shoichi Haimoto, ${ }^{1,2}$ Yusuke Nishimura, ${ }^{2}$ Masabito Hara, ${ }^{3}$ Toshibiko Wakabayashi, ${ }^{2}$ Howard Ginsberg. ${ }^{1}$ From the 'Division of Neurosurgery, St. Michael's Hospital, University of Toronto, Toronto, Ont.; ${ }^{2}$ Department of Neurosurgery, Nagoya University Graduate School of Medicine, Nagoya, Japan; ${ }^{3}$ Department of Neurosurgery, Inazawa Municipal Hospital, Inazawa, Japan

Background: Recent studies have shown the efficacy of subfascial intrawound application of vancomycin powder in spine surgery to reduce the rate of surgical site infections (SSIs). However, to date, no study has evaluated the efficacy and safety of suprafascial application of vancomycin powder in spine surgery. The purpose of this study was to quantify the rate of SSIs after open instrumented posterior spinal fusion with and without application of suprafascial vancomycin powder, and to evaluate the rate of vancomycin powder-related local adverse effects. Methods: We conducted a single-centre retrospective case-control study involving adult patients who underwent open instrumented posterior fusion of the cervical, thoracic or lumbar spine conducted by a single surgeon from January 2010 through December 2016.
In March 2013, routine application of $1 \mathrm{~g}$ of suprafascial vancomycin powder was started for all cases in addition to standard systemic antibiotic prophylaxis. Baseline demographics, operative data and SSI rates were compared between the study groups. The incidence of vancomycin powder-related adverse effects was analyzed. Results: A total of 515 patients (268 in the untreated group and 247 in the treated group) were included in the study. The mean age was significantly higher in the treated group than in the untreated group (58.4 v. $54.4 \mathrm{yr}, p<0.01)$. Operative variables were similar between the study groups. Patients who received vancomycin powder had a significantly lower infection rate $5.6 \%$ in the untreated group v. $0 \%$ in the treated group, $p<$ $0.001)$. No vancomycin powder-related adverse effects were identified in the treated group. Conclusion: Routine application of suprafascial intrawound vancomycin powder in addition to systemic antibiotic prophylaxis is an easy-to-use, safe and effective strategy for preventing SSIs after instrumented posterior spinal fusion. Suprafascial application of vancomycin powder could be a valuable alternative to previously reported subfascial distribution by minimizing the risk of local adverse drug reactions.

\section{4}

Increased risk of infections following blood transfusions in anterior cervical spine surgery. Hussam Fabri, ${ }^{1}$ Stephen Kingwell, ${ }^{1,2,3}$ Mabmoud Bedaiwy, ${ }^{1}$ Mobammed Al-Sawat, ${ }^{1}$ Abdulkareem Al-Rabie, ${ }^{1}$ Yeye AlQatbani, ${ }^{1}$ Saad Surur, ${ }^{1}$ Philippe Phan, 1,2,3 Eve Tsai,, 1,3 Safraz Mobammed, ${ }^{1,4}$ Mobamad Hoda, ${ }^{1}$ Darren Roffey, ${ }^{1,3}$ Eugene Wai. ${ }^{1,2,3}$ From the ${ }^{1}$ Ottawa Spine Collaborative Analytics Network (OSCAN), The Ottawa Hospital, Ottawa, Ont.; ${ }^{2}$ Division of Orthopaedic Surgery, University of Ottawa, The Ottawa Hospital, Ottawa, Ont.; ${ }^{3}$ Clinical Epidemiology Program, Ottawa Hospital Research Institute, Ottawa, Ont.; ${ }^{4}$ Division of Neurosurgery, University of Ottawa, The Ottawa Hospital, Ottawa, Ont.

Background: Studies in transfusion of allogeneic blood have shown an immunomodulatory effect that may increase the risk of infection. Spinal surgery is considered to have a relatively elevated risk of blood loss with increased associated transfusion requirements owing to bone and epidural bleeding. To date, there have been few studies on the issue of postoperative infections following transfusions in cervical spinal surgery. Methods: We performed a retrospective study using the American College of Surgeons National Surgical Quality Improvement Program (ACS NSQIP) database. Data from patients who underwent elective anterior cervical spinal surgery between 2011 and 2014 were obtained. Outcomes included factors associated with superficial wound infection, urinary tract infection (UTI), deep wound infection, pneumonia, organ space surgical site infection (SSI), sepsis and wound dehiscence. Multivariate logistic regression analysis was used to adjust for any factor that was associated with infection in univariate analysis. Results: A total 12623 patients were identified, resulting in data from 55 patients who had postoperative transfusions $(0.4 \%)$, and 240 patients who had infections $(1.9 \%)$. In univariate analysis, transfusion was associated with increased odds of infection (odds ratio $[\mathrm{OR}]=13.4,95 \%$ confidence interval [CI] 6.9-26.4). Multivariate logistic regression analysis with adjustment for confounding factors showed further increased odds of infection following transfusion (adjusted 
$\mathrm{OR}=14.9,95 \%$ CI 1.1-187.5). Additional subgroup analyses indicated that duration of surgery was not an interacting factor. Conclusion: Allogeneic blood transfusion was significantly associated with increased risk of infection in elective anterior cervical spinal surgeries. This supports the immunomodulatory hypothesis, and suggests that interventions to reduce blood loss and transfusion may reduce postoperative infections. However, caution should be applied in inferring any causative effect owing to the prospect of other unknown confounding factors.

\section{7}

Allogeneic blood transfusions and infection risk in singlelevel lumbar posterior decompression: an American College of Surgeons National Surgical Quality Improvement Program study. Amedeo Falsetto, 1,2 Darren Roffey, ${ }^{1,3}$ Mobamad Hoda, ${ }^{1}$ Stephen Kingwell, ${ }^{1,2,3}$ Philippe Phan, ${ }^{1,2,3}$ Alexandra Stratton, ${ }^{1,2}$ Eugene Wai. ${ }^{1,2,3}$ From the ${ }^{1}$ Ottawa Spine Collaborative Analytics Network (OSCAN), Ottawa, Ont.; ${ }^{2}$ Division of Orthopaedic Surgery, University of Ottawa, The Ottawa Hospital, Ottawa, Ont.; ${ }^{3}$ Clinical Epidemiology Program, Ottawa Hospital Research Institute, Ottawa, Ont.

Background: Lumbar spine fusion surgery has postoperative blood transfusion rates as high as $30 \%$. Recent studies indicate American Society of Anesthesiologists class > 3, bleeding disease, multilevel surgery and presurgical blood urea nitrogen are predictors of transfusion for lumbar spine surgery. It is hypothesized that transfusions lead to an immunomodulatory effect, resulting in a relatively immunocompromised state, which can then result in developing surgical site infections (SSIs), urinary tract infections (UTIs) and overall postoperative infections. Our objective was to explore to what extent transfusions are associated with postoperative infections while adjusting for existing comorbidities. Methods: We performed a retrospective review of prospectively collected data in the American College of Surgeons National Surgical Quality Improvement Program (ACS NSQIP) database from 2006 to 2014. Patients with primary single-level lumbar posterior decompression for an elective degenerative lumbar condition were identified. Patients with cancer, emergency surgery, dialysis or renal failure, paralysis or pre-existing spine infection were excluded. Data were analyzed using a multivariate logistic regression model. Results: A total of 29598 patients were identified, $1480(5 \%)$ of whom underwent a transfusion. Older age, female sex, lower preoperative albumin, weight, higher ASA class, duration of surgery and smoking were significantly associated with transfusions $(p<0.05)$. Preoperative hematocrit (HCT) was not associated with transfusion $(p=0.28)$. There were $141(0.5 \%)$ SSIs and $451(1.5 \%)$ total postoperative infections in any body region. The odds of SSI and total infections were 3.0 times greater $(95 \%$ confidence interval [CI $1.8-4.8$ ) and 2.1 times greater $(95 \% \mathrm{CI}$ 1.5-2.9), respectively, in patients who had a transfusion. Multivariate logistic regression analysis, adjusting for all of the above noted risk factors and HCT, showed adjusted odds ratios of 2.5 (95\% CI 1.5-4.1) and 1.9 (95\% CI 1.4-2.7), respectively, for SSI and total infections. Conclusion: Transfusions in patients who underwent single-level lumbar posterior decompression surgery were independently associated with SSIs and total infections. Identification of risk factors for transfusion may be helpful in the perioperative optimization strategies for decreasing transfusions, which may in turn lower the risk of SSI and total infection overall.
133

Canadian national survey on surgical site infection prophylactic strategies in spine surgery. Reina Yao, ${ }^{1}$ Mark $\mathrm{Xu},{ }^{2}$ Michael Goytan, ${ }^{2}$ Fobn Street. ${ }^{1}$ From the ${ }^{1}$ University of British Columbia, Vancouver, BC; ${ }^{2}$ University of Manitoba, Winnipeg, Man.

Background: Numerous prophylactic strategies have been proposed to mitigate risk of surgical site infection (SSI) . However, no national guidelines exist and practices of Canadian spine surgeons remain unknown. We conducted a Canadian Spine Society (CSS) survey to capture current practices with respect to SSI prophylaxis. Methods: We emailed the CSS membership a 37-item questionnaire developed after a systematic review of the literature. Results: A total of 52 spine surgeons completed the survey, of whom $94 \%$ were fellowship trained. With the exception of preoperative antibiotics in instrumented cases, no SSI prevention strategies were universally used by respondents. For all prophylactic strategies, there was greater reported use for instrumented cases as compared with uninstrumented. Strategies used by most respondents were methicillin-resistant Staphylococcus aureus (MRSA) screening (64\% in instrumented and $59 \%$ in uninstrumented cases), optimization of diabetic blood glucose $(69 \%$ and $60 \%)$, hair clipping from the operative site $(86 \%$ and $83 \%)$, iodine-impregnated drape application in instrumented cases $(60 \%)$, routine Cell Saver use, saline wound irrigation (97\% and 93\%), intrawound vancomycin in instrumented cases $(66 \%)$ and administration of antibiotics before and after surgery. All respondents gave preoperative antibiotics in instrumented cases, with $95 \%$ in uninstrumented cases, and almost all used a 1st generation cephalosporin. Ninety percent redosed intraoperatively every 4 hours. In cases of penicillin allergy, there was equal use of vancomycin and clindamycin. Seventy-eight percent gave postoperative antibiotics in instrumented cases compared with $53 \%$ in uninstrumented cases, with $87 \%$ of respondents giving 24 hours' duration or less. Sixy-seven percent of respondents closed with monofilament subcuticular absorbable suture, whereas $43 \%$ used staples. Factors considered high risk by most respondents were obesity, diabetes, immunodeficiency, history of infection, revision surgery, tumour cases, instrumentation and prolonged surgical duration. Conclusion: There is significant variation in the adoption of SSI prophylactic strategies by CSS members. This survey will help direct future development of national guidelines for spine SSI prophylaxis.

\section{8}

Determining the extent of clinical practice variation across Canada for single-level posterior surgery for lumbar degenerative spondylolisthesis. Vinay Kulkarni, ${ }^{1}$ Kunal Bbanot, Albert Yee, ${ }^{1}$ Greg McIntosh,${ }^{2}$ Henry Abn, ${ }^{1}$ Jeremie Larouche. ${ }^{1}$ From the ${ }^{1}$ University of Toronto, Toronto, Ont.; ${ }^{2}$ Canadian Spine Outcomes and Research Network, Toronto, Ont.

Background: There is considerable practice debate and discordant literature as to the optimal surgical treatment for degenerative lumbar spondylolisthesis. Single-level posterior surgery for this condition is typically associated with excellent patientreported outcomes. This study aims to determine the clinical practice variation in surgical treatment that exists across Canada for this patient population. Methods: A review of prospectively collected data from the Canadian Spine Outcomes and Research 
Network (CSORN) registry was performed (enrolled patients October 2008 to August 2017). Inclusion criteria were patients with a primary diagnosis of degenerative lumbar spondylolisthesis, a chief complaint of radiculopathy or claudication and treatment with a single-level posterior lumbar spinal decompression with or without fusion. Statistical analyses were performed to evaluate surgical practice variability, including the following parameters: with or without fusion, the use of medical devices including interbody devices, type of bone grafting, and intraoperative use of surgical navigation and neuromonitoring. We evaluated provincial differences in surgical practice and compared surgery based on surgeon background specialty training (i.e., neurosurgery or orthopedics). Results: We included 244 patients, of whom 117 (48\%) received decompression alone and $127(52 \%)$ received decompression with fusion. Mean patient age at surgery was 67 years, and $62 \%$ of patients were female. Intraoperative neuromonitoring was used in $9 \%$ of cases $(n=21)$. In fusion procedures, allograft was used in $35 \%$ of cases $(n=44)$, synthetic graft in $10 \%(n=13)$ and surgical navigation in $30 \%$ $(n=38)$. There was no statistical difference in fusion rates comparing background surgeon specialty training; however, orthopedic surgeons were 6 times more likely to perform a transforaminal lumbar interbody fusion (TLIF; odds ratio 6.3, $p<0.001$ ). There was significant practice variability between Canadian provinces when considering the use of surgical fusion, TLIF and bone allograft $(p<0.05)$. Conclusion: There is considerable clinical practice variation across Canada in surgery performed for singlelevel degenerative lumbar spondylolisthesis. This variability is important as both care providers and health system funders consider value-based surgery. Our research motivates ongoing comparative study that should consider patient-reported outcome measures after surgery.

\section{8}

Use of the Spine Adverse Events Severity System (SAVES) in patients undergoing spine surgery not related to trauma. A comparison with National Surgical Quality Improvement Program for the identification of acute care adverse events. Fennifer Lauren Nevin, ${ }^{1}$ Étienne Bourassa-Moreau, ${ }^{2}$ John Street. ${ }^{3}$ From the ${ }^{1}$ Department of Orthopaedics, University of British Columbia, Vancouver, BC; ${ }^{2}$ Oxford Spinal Unit, Oxford University Hospitals, Oxford, United Kingdom; ${ }^{3}$ Blusson Spinal Cord Centre, Division of Spine, Department of Orthopaedics, University of British Columbia, Vancouver, BC.

Background: We sought to compare the Spine Adverse Event Severity System (SAVES) with the National Surgical Quality Improvement Program (NSQIP) for the reported incidence of adverse events and per patient rate of adverse events, and to identify risk factors for adverse events for adult patients undergoing major spine surgery. Methods: In this observational cohort comparison, patients who underwent major spine surgery other than for trauma at a quarternary care spine program between 2011 and 2016 were identified using Current Procedural Terminology codes in the NSQIP database and matched to corresponding records in SAVES. The NSQIP adverse events were mapped to appropriate ones in SAVES. Results: There were 672 patients identified in NSQIP; 635 also had complete records in SAVES. The SAVES system identified 7.8 times as many individual adverse events than the NSQIP database (overall incidence of adverse events 2188 v. 277) and more patients who suffered an adverse event (SAVES 357, NSQIP 191). Intraoperative events were identified in only $0.94 \%$ of patients in NSQIP as compared with $19.5 \%$ in SAVES. Many clinically important adverse events were identified more often in SAVES, including superficial surgical site infection (1.3 times more, $p=0.029)$, pneumonia ( 4.5 times more, $p<0.0001)$, pulmonary embolus (3.2 times more, $p<$ $0.0001)$, UTI (3.2 times more, $p<0.0001)$ and sepsis $(1.9$ times more, $p<0.0001)$. Risk factors such as age and surgery duration were correlated with adverse events collected by both SAVES and NSQIP. Conclusion: Using the SAVES system identified more patients who suffered adverse events and more events per person than the NSQIP database. This study shows that using a prospective and validated specialty-specific adverse event database more reliably identifies clinically important adverse events, which may influence quality improvement initiatives.

\section{5}

The effect of perioperative adverse events on long-term patient-reported outcomes after lumbar spine surgery. Oliver Ayling, Charles Fisher, Hanbing Zhou, Michael Bond, Tamir Ailon, Nicolas Dea. From the University of British Columbia, Vancouver, BC.

Background: Perioperative adverse events lead to patient disappointment, resource use and increased health care costs. There is a paucity of data as to how complications affect long-term patient reported outcomes (PROs). The purpose of this study is to examine perioperative adverse events and their long-term impact on PROs. Methods: We analyzed data from 3621 consecutive patients who underwent elective spine surgery for degenerative lumbar spine disorders enrolled in the Canadian Spine Outcomes and Research Network (CSORN) prospective database. Peri- or immediate post-operative adverse events were divided into major and minor for lumbar disc herniation, degenerative spondylolisthesis and spinal stenosis. Outcomes at 3 and 12 months on the Oswestry Disability Index (ODI), visual analog scales for leg and back, and short-form 12 (SF-12) were assessed using univariate and multivariate linear regression. Results: A total of 109 major complications occurred (3.01\%). Patients who had major complications were significantly older $(64.41 \pm 11.59 \mathrm{yr}$ v. $57.26 \pm$ $14.87 \mathrm{yr}$ ), were more likely to have had their condition for $>2$ years (47.7\% v. $32.85 \%)$, had longer surgeries (177.6 v. $134.3 \mathrm{~min}$ ) and had higher rates of comorbidities (all $p<0.05$ ). Major complications were associated with higher ODI at 3 months $(p=0.015)$ but not at 1 year $(p=0.7)$, as well as lower SF-12 Physical Component score at 3 months $(p=0.005)$, but not at 1 year $(p=0.16)$. Length of stay was longer after an adverse event $(12.15 \pm 20.7 \mathrm{~d}$ v. $3.72 \pm 16.9 \mathrm{~d}, p<0.0001)$. In multivariate linear regression, after controlling for significant variables on univariate analysis, no single adverse event was identified as a predictor of a poorer ODI at 3 months. Conclusion: Major adverse events during hospital admission lead to significantly worse outcomes at 3 months, but not at 1 year, after surgery for degenerative lumbar disease.

\section{7}

Surgical satisfaction and clinical outcomes in degenerative spondylolisthesis. Michael Craig, ${ }^{1}$ Tamir Ailon. ${ }^{1,2}$ From the ${ }^{1}$ Division of Neurosurgery, University of British Columbia, 
Vancouver, BC; ${ }^{2}$ Vancouver Spine Surgery Institute, Vancouver, BC.

Background: Two emerging themes in health care are valuebased delivery and patient-centred care. Accordingly, patient satisfaction has drawn increasing attention as an important outcome of elective surgical care. We sought to assess the strength of association between patient satisfaction and surgical outcomes in a population of patients with degenerative spondylolisthesis. Methods: Patients were retrospectively identified from the Canadian Spine Outcomes Research Network (CSORN) database. Patients with radiographic evidence of degenerative spondylolisthesis who underwent surgery for neurogenic claudication or radiculopathy were included. As part of CSORN, patients completed questionnaires during initial assessment and 618 weeks postoperatively to indicate levels of back and leg pain, and completed validated outcome questionnaires including the short-form 12 (SF-12) and Oswestry Disability Index (ODI). Postoperatively, patients responded to "Are you satisfied with the results of your spine surgery?" using a 5 -point scale. Correlation coefficients and 1-way analysis of variance were performed between clinical outcomes and patient satisfaction. Results: We identified 85 patients, all of whom underwent lumbar discectomy, laminectomy or arthrodesis. The population was $25 \%$ male, with a mean age of 65.2 (SD 8.5) years. All clinical outcome measures improved after surgery, and $90 \%$ of patients were extremely or somewhat satisfied with their result. The change in leg pain $(r=$ $0.63)$, and absolute postoperative back pain $(r=0.65)$ correlated most strongly with patient satisfaction, compared with change in scores for the SF-12 $(r=0.32)$ and ODI $(r=0.53)$. Mean improvement in pain scores differed by degree of satisfaction. For example, "somewhat dissatisfied" patients had a mean improvement in leg pain of 1.5 points (standard deviation [SD] 2.1), whereas "extremely satisfied" patients had a mean improvement of 5.9 points (SD 3.0). Conclusion: Specific symptoms such as back and leg pain correlated more strongly with patient satisfaction than more global outcome measures. This may suggest that patient satisfaction is more influenced by pain relief than by improvements in health-related quality of life measures.

\section{1}

Spinopelvic parameters and sagittal balance do not correlate with baseline pain, function or general health in degenerative lumbar spondylolisthesis. Michael Staudt, ${ }^{1}$ Charles Fisher, ${ }^{2}$ Raja Rampersaud, ${ }^{3}$ Andrew Glennie, ${ }^{4}$ Ken Thomas, ${ }^{5}$ Raphaële Charest-Morin, ${ }^{6}$ Edward Abrabam, ${ }^{7}$ Sean Christie, ${ }^{4}$ Nicolas Dea, ${ }^{8}$ Greg McIntosh, ${ }^{9}$ Zennifer Urquhart, ${ }^{1}$ Christopher Bailey. ${ }^{1}$ From the ${ }^{1}$ Western University, London, Ont.; ${ }^{2}$ University of British Columbia, Vancouver, BC; ${ }^{3}$ University of Toronto, Toronto, Ont.; ${ }^{4}$ Dalhousie University, Halifax, NS; ${ }^{5}$ University of Calgary, Calgary, Alta.; ${ }^{6}$ Université Laval, Laval, Que.; ${ }^{7}$ Dalhousie University, St. John, NB; ${ }^{8}$ Université de Sherbrooke, Sherbrooke, Que.; ' $\mathrm{CBI}$ Health Group, Toronto, Ont..

Background: The importance of sagittal balance and spinopelvic parameters are recognized in the management and outcomes of adult spinal deformity and isthmic spondylolisthesis, but less is known regarding their role in degenerative lumbar spondylolisthesis (DLS). The purpose of this study was to determine the influence of baseline sagittal balance and spinopelvic parameters on pain, function and health-related quality of life (HRQOL) of patients presenting with DLS. Methods: Patients with DLS were enrolled in a prospective, multicentre Canadian Spine Outcomes Research Network (CSORN) study. Baseline patient demographics, radiographic spinopelvic parameters and sagittal alignment were analyzed for possible correlation to HRQOL outcomes (numerical rating scale [NRS] for back or leg pain, Oswestry Disability Index [ODI], short-form 12 [SF-12], Patient Health Questionnaire [PHQ-9] and EQ-5D) using Pearson correlations. Results: We analyzed data from 154 patients (mean age $65.8 \mathrm{yr}$, mean body mass index 29.1 ) with grade I (72\%) or II (28\%) DLS, of whom 95 were female. Primary symptoms were related to neurogenic claudication $(80 \%)$, radiculopathy $(15.3 \%)$ or back pain (4.7\%). Mean symptom duration was more than 2 years in $71 \%$ of patients. Patients had moderate to severe symptoms and disability (mean NRS back 7.2, NRS leg 7.3, ODI 45.6, SF-12 physical component 33.2, SF-12 mental component 49.6, EQ-5D 0.54). Baseline measurements of radiographic alignment included sacral slope (SS; $34.9^{\circ} \pm 16.7^{\circ}$ ), pelvic tilt $\left(\mathrm{PT} ; 24.8^{\circ} \pm 9.5^{\circ}\right.$ ), pelvic incidence (PI; $57.4^{\circ} \pm 13.8^{\circ}$ ), sagittal vertical axis (SVA; $35.8 \pm$ $40.8 \mathrm{~mm}$ ), lumbar lordosis (LL; $45.7^{\circ} \pm 13.6^{\circ}$ ), thoracic kyphosis (TK; $36.8^{\circ} \pm 13.0^{\circ}$ ), T9-spinopelvic inclination (T9SPI; 9.62 \pm $4.0^{\circ}$ ) and T1-spinopelvic inclination (T1SPI; $4.43^{\circ} \pm 10.4^{\circ}$ ). There were $54 \%$ of patients who had an insufficient lumbar lordosis based on the relationship between PI and LL (LL < PI $-9^{\circ}$ ). Sagittal vertical axis correlated with T9SPI, LL, PI and PT; whereas T9SPI correlated with SVA, LL, TK, and T1SPI. There was no correlation between baseline patient outcomes and spinopelvic parameters and alignment. Conclusion: Patients with DLS have global sagittal misalignment compared with published normative controls, highlighted by less lumbar lordosis, a PI to LL mismatch, and increased PT. Spinopelvic alignment was not associated with patient outcomes, although positive sagittal balance may also be influenced by spinal stenosis.

\section{2}

Does back pain improve in surgically treated degenerative lumbar spondylolisthesis: What can we tell our patients? Michael Bond, ${ }^{1}$ Hanbing Zbou, ${ }^{1}$ Chris Bailey, ${ }^{2}$ Nicolas Dea, ${ }^{1}$ Raphaele Charest-Morin, ${ }^{3}$ Andrew Glennie, ${ }^{4}$ Neil Manson, ${ }^{5}$ Hamilton Hall, ${ }^{6}$ Ken Thomas, ${ }^{7}$ Raja Rampersaud, ${ }^{8}$ Greg McIntosh, ${ }^{9}$ Charles Fisher. ${ }^{1}$ From the ${ }^{1}$ Combined Neurosurgical and Orthopaedic Spine Program, Vancouver General Hospital and University of British Columbia, Vancouver, BC; ${ }^{2}$ Division of Orthopaedics, Department of Surgery, Western University, London, Ont.; ${ }^{3}$ Department of Orthopaedics, Centre Hospitalier Universitaire de Québec, Québec, Que.; ${ }^{4}$ Department of Surgery, Dalhousie University, Halifax, NS; ${ }^{5}$ Canada East Spine Centre, Saint John Regional Hospital, Saint John, NB; ' ${ }^{6}$ Department of Surgery, University of Toronto, Toronto, Ont.; ${ }^{7}$ Departments of Surgery and Clinical Neurosciences, University of Calgary, Calgary, Alta.; ${ }^{8}$ Department of Surgery, Division of Orthopedic Surgery, Toronto Western Hospital, Toronto, Ont.; ${ }^{9}$ Canadian Spine Society Research Operations, Markdale, Ont.

Background: Surgery for degenerative lumbar spondylolisthesis (DLS) has traditionally been indicated for patients with neurogenic 
claudication and radicular pain. Surgery improves patients' disability and lower extremity symptoms, but little is known about the impact on back pain. This study aims to evaluate changes in back pain after surgery and identify prognostic factors influencing these changes in surgically treated DLS. Methods: Consecutive patients with surgically treated DLS were prospectively enrolled in the Canadian Spine Outcomes Research Network (CSORN) registry. Patients had demographic data, clinical information, disability (Oswestry Disability Index [ODI]) and back pain (numerical rating scale) scores collected prospectively at baseline and at 3, 12 , and 24 months' follow-up. Results were compared with baseline using simple summary statistics, and factors associated with improved back pain were assessed with multivariable regression $(p<0.05)$. Results: A total of 557 patients were identified, all of whom had reached 3 months' follow-up, 376 (67.5\%) of whom had reached 12 months' follow-up and 141 (25.3\%) of whom had reached 24 months' follow-up. Mean age at baseline was $66.2 \pm$ 9.5 years, and $58.5 \%$ of patients were female. Back pain improved significantly at 3 months, and was maintained at 12 and 24 months' follow-up compared with baseline $(p<0.001)$. Improvement in back pain scores at 12 months was a mean of $3.2 \pm 2.8$ points, and clinically significant improvement in back pain was seen in $75 \%$ of patients (minimal clinically important difference was 2 points). Factors associated with worse back pain scores at 12 months' follow-up were higher baseline back pain, worse baseline physical function, at least 1 postoperative adverse event and longer duration of symptoms $(p<0.05)$. Conclusion: Back pain improved significantly during follow up for patients who received surgical treatment for DLS. This research shows that for patients who undergo surgery for DLS, most will have improved back pain at 1-year's follow-up.

\section{3}

Slip progression in low-grade degenerative lumbar spondylolisthesis and symptomatic deterioration after minimally invasive decompression. Robert Ravinsky, ${ }^{1,2}$ Eric Crawford, ${ }^{1,2}$ Raja Rampersaud. ${ }^{1,2}$ From the ${ }^{1}$ Division of Orthopaedic Surgery, University of Toronto, Toronto, Ont.; ${ }^{2}$ Division of Orthopaedic Surgery, Toronto Western Hospital, Toronto, Ont.

Background: Our objective was to determine whether there is a relationship between slip progression and symptomatic worsening after decompression without fusion for low-grade degenerative lumbar spondylolistheses (DLS). Methods: We performed a retrospective review (January 2008 to December 2016) of 1- to 2-level minimally invasive surgical (MIS) decompression for grade I-II DLS. We included patients who had a minimum of 1 year of follow-up with prospectively collected baseline and follow-up Oswestry Disability Index (ODI) scores. Slip percentage was measured on baseline and follow-up radiographs using Surgimap software. Results: We identified 60 patients (35 women $[58.3 \%]$ ), with a mean age of 65.7 (standard deviation [SD] 9.83; range 46-85) years who met the inclusion criteria. Mean length of follow-up was 1.7 (SD 0.4; range 1-2.4) years. Spondylolisthesis slip percentage increased in $56.7 \%(34 / 60)$ of patients. Slip percentage increased significantly $(p=0.001)$ from baseline (mean 16.9\% [SD 1.02\%]) to follow-up (mean $19.7 \%$ [SD 1.2\%]). A logistic regression model comparing stable with progressive slip percentages identified that women were more likely to have progressive slips compared with men (odds ratio $7.08,95 \%$ confidence interval 2.0-24.8; $p=0.002$ ). Seventy-four percent of women (26/35) had progressive slips compared with $32 \%$ of men $(8 / 25)$. ODI scores improved in $90 \%(54 / 60)$ of patients. Oswestry Disability Index scores decreased $(p<0.0001)$ from baseline to follow-up, with a mean decrease of 21.4 (SD 2.4) points. Oswestry Disability Index scores and spondylolisthesis slip percentage did not correlate at baseline $(p=0.45)$ or at follow-up $(p=0.42)$, nor did change in ODI scores and change in slip percentage ( $p=0.47)$. Additionally, changes in ODI scores were not different between patients with stable slips compared with those with progressive slips at follow-up $(p=0.6)$. Furthermore, of the 34 patients with slip progression, there was no difference in changes in ODI score $(p=0.5)$ for those with $1 \%-5 \%$ progression (15/34) compared with those with more than $5 \%$ slip progression (19/34). Conclusion: Slip progression was more likely to occur in women. Despite a small degree of slip progression in most patients, there was no correlation with symptom worsening as measured by the ODI.

\section{5}

Lumbar spine fusion: the influence of evidence on decision-making. Daniel Yavin, ${ }^{1,2}$ Nathalie fette, ${ }^{1,2,3,4}$ Samuel Wiebe, ${ }^{1,2,3,4}$ Thomas E. Feasby, ${ }^{1,2,3,4}$ R. Fobn Hurlbert, ${ }^{5}$ Andrew Nataraj, ${ }^{6}$ Garnette R. Sutberland, ${ }^{1,3}$ Perry Dhaliwal, ${ }^{7}$ Stephan du Plessis, ${ }^{1}$ Steven Casba. ${ }^{1,3}$ From the ${ }^{1}$ Department of Clinical Neurosciences, University of Calgary Cumming School of Medicine, Calgary, Alta.; ${ }^{2}$ Department of Community Health Sciences, University of Calgary Cumming School of Medicine, Calgary, Alta.; ${ }^{3}$ The Hotchkiss Brain Institute, University of Calgary Cumming School of Medicine, Calgary, Alta.; ${ }^{4}$ The O'Brien Institute for Public Health, University of Calgary Cumming School of Medicine, Calgary, Alta.; ${ }^{5}$ Division of Neurosurgery, Department of Surgery, University of Arizona, Tucson, Ariz.; ${ }^{6}$ Department of Surgery, University of Alberta, Edmonton, Alta.; ${ }^{7}$ Section of Neurosurgery, Department of Surgery, University of Manitoba, Winnipeg, Man.

Background: Evaluation of the determinants of widely varying regional rates of lumbar fusion is needed. Although patient-, surgeon-, and setting-specific factors have been investigated, the association between decision-making and the quality of the underlying evidence remains unclear. This study therefore aimed to evaluate the influence of the quality of evidence on experts' decisions to perform lumbar fusion for degenerative indications. Methods: The quality of evidence underlying 1296 distinct degenerative indications for lumbar fusion was graded according to criteria developed by the North American Spine Society. A North American expert panel of 13 physicians was convened, and members were provided with scenario-specific evidence grades and a systematic literature review that evaluated the safety and efficacy of lumbar fusion. Using a modified Delphi process, panel members rated the appropriateness of lumbar fusion for each indication. The strength of association between expert panel disagreement and scenarios' clinical characteristics, radiologic findings, and levels of evidence was assessed. Results: In the 1296 scenarios evaluated, $13 \%$ were supported by level I evidence, $33 \%$ by level II evidence, and $54 \%$ by level III evidence. Indications involving spondylolisthesis were associated with the highest 
percentage of level I evidence. Increasing quality of evidence was not associated with decreasing disagreement when panelists considered lumbar fusion for spinal stenosis $(p=0.11)$, spondylolisthesis $(p=0.79)$, disc herniation $(p=1.0)$, or spondylosis $(p=$ $0.63)$. In contrast, disagreement among panelists was dependent on the extent of patients' disability $(p<0.001)$, form of low back pain $(p<0.001)$, severity of neurologic signs and symptoms $(p<$ $0.001)$, and radiologic findings $(p<0.001)$. Conclusion: The quality of underlying evidence for lumbar fusion did not account for differing surgical decision-making. The reliance on level III evidence for the majority of surgical indications may limit the use of the available literature in patient care decisions. (Funded by the Alberta Spine Foundation.)

\section{6}

An exploratory analysis of spine patients' preoperative concerns and decision-making process: Does what the surgeon say matter? Norab-Faye Matthies, Sam Keshen, Stephen Lewis, Fiona Webster, Antbony Perruccio, Raja Y. Rampersaud. From the University of Toronto, Toronto, Ont.

Background: Surgical consultation is an important step for patients considering elective surgery. Despite proven effectiveness of many spine procedures, patients who are good operative candidates may choose nonsurgical treatment. Our goal is to determine what factors affect a patient's decision to undergo elective spine surgery following surgical consultation. Methods: Survey and open-response questions regarding pre- and postconsultation (PRE and POST) surgical concerns and willingness to undergo surgery were analyzed from 124 patients with nonurgent spine disorders who were deemed surgical candidates. Demographic, surgical willingness, and patient concern rank data were analyzed. Free-text data were tallied and coded using line-by-line analysis for overarching themes. Subanalysis was included on patients who reconsidered their surgical willingness POST. Results: Presurgical concerns were grouped into 6 major headings: interference on quality of life (QOL), general fear of surgery or complications, physical concerns (pain or functional restriction), surgical success, risk, and concerns about the surgeon. Of 124 patients, 174 concerns were raised PRE and 364 POST. Success and risk were the highest coded PRE concerns $(48,45)$ and remained equally as concerning POST $(71,81)$; Fear, physical concerns, and QOL all more than doubled POST. Of the postoperative concerns, risk was most highly prioritized, followed by success and QOL. Subanalysis of the impact of surgical consultation on willingness to undergo surgery demonstrated 103 remained willing PRE and POST and 6 became unwilling. Twenty-one patients were unwilling before consultation; only 5 of these remained unwilling. A small analysis of freetext concerns on these patients concluded that success, risk, and physical concerns were most important; social implications of surgery and a desire to explore alternative therapies were emphasized. There were no differences in PRE concerns between willing and unwilling patients. Conclusion: The decision to undergo surgery is multifactorial and complex. Eighty-seven percent of patients have made up their mind before attending their surgical consultation. An appropriate, contextually specific understanding of a patient's surgical willingness and concerns should be pursued to enable discussion and aid in a shared decision-making process.
39

Clinical practice variation (CPV) in informed consent for lumbar spine surgery. Kunal Bhanot, ${ }^{1}$ Eric Crawford, ${ }^{1,2}$ Gary Rosenberg, ${ }^{3}$ Zachary Tan, ${ }^{3}$ Albert Yee. ${ }^{1,4}$ From the ${ }^{1}$ Division of Orthopaedic Surgery, University of Toronto, Toronto, Ont.; ${ }^{2}$ Institute of Health Policy, Management and Evaluation, University of Toronto, Toronto, Ont.; ${ }^{3}$ University of British Columbia, Vancouver, BC; ${ }^{4}$ Sunnybrook Health Sciences Centre, Toronto, Ont.

Background: Informed consent is a critical tenet of the physician-patient relationship. We hypothesize that there is significant clinical practice variation (CPV) in the consent process discussion between surgeons and their patients. We standardized a routine single-level lumbar microdiscectomy surgical case to determine the extent of CPV that exists amongst practising spine surgeons in Canada. Methods: An established framework for surgical complications in routine posterior lumbar single-level spinal microdiscectomy was used. A cross-sectional survey was administered to Canadian Spine Society members with surgical expertise in this procedure. Fifteen routinely identified surgical risks were surveyed and graded as commonly, uncommonly, rarely or not routinely discussed by surgeons. Results: A mean of 3.6 (24\%) out of 15 complications were "not routinely discussed" by the surgeons surveyed. Interestingly, "wrong level surgery" was a complication not discussed by $59.2 \%$ of respondents (29/49), lower than we anticipated. In addition, orthopedic surgeons were more likely to discuss fewer complications $(4.1 / 15 ; 27.3 \%)$ as compared with their neurosurgical counterparts $(2.2 / 15 ; 14.7 \%$; $p=0.03)$. Poisson multivariable regression considering surgeon and practice factors indicated that surgeon specialty (neurosurgeons compared with orthopedic surgeons, $p=0.02$ ) and the experience in number of consent discussions $(<50 \mathrm{v}$. $>50, p=$ 0.03 , and $<50$ v. $100, p=0.006)$ were statistical predictors. Orthopedic surgeons were more likely to discuss fewer complications compared with neurosurgeons, and physicians with more experience obtaining consent were also more likely to discuss fewer complications. Conclusion: There is significant CPV in the individual risks discussed during the formal informed consent process. The results of this study highlight the importance of considering CPV. Surgeon background training and their experience with informed consent appear to be predictive of potential complications that may be discussed with patients. Although we recognize that in many situations individualization of informed consent is required because of patient and anatomic factors, in this survey, we did standardize a "routine" clinical scenario for which there still appears to be significant CPV.

\section{0}

Biological and clinical outcomes of chiropractic spinal manipulative therapy in the treatment of acute inflammatory radiculopathy secondary to lumbar disc herniation: a pilot study. Paul Bishop, Feffrey Quon, Brian Arthur, Melissa Nadeau, Nicolas Dea, Tamir Ailon, Charles Fisher, Marcel Dvorak, Scott Paquette, Brian Kwon, Fobn Street. From the Division of Spine, Department of Orthopaedics, University of British Columbia, Vancouver, BC

Background: We performed a pilot evaluation of a randomized controlled trial (RCT) of the effects of chiropractic spinal 
manipulative therapy (CSMT) on in vivo inflammatory cytokine (IC) expression and clinical outcomes in patients with acute sciatica and lumbosacral disc herniation (AS/LDH). Methods: Patients with AS/LDH of less than 16 weeks' duration were randomized to either 6 weeks of CSMT or the surgical wait list with no further nonoperative physical therapy. Standardized neurologic and lumbar spine examinations, visual analog scale (VAS) and Roland Morris Disability scores, in addition to serum IC levels were assessed at baseline and at 6, 12 and 24 weeks. Inflammatory cytokines were also measured in disc tissue, nerve root and disc lavagate collected during surgery. Research ethics approval was obtained. Results: A total of 40 of $44(91 \%)$ invited patients participated and were randomized. Of these patients, 13 avoided surgery. Post-CSMT improvement was associated with longer duration of sciatica, absence of crossover sign and motor deficit, reduced lumbar flexion increment and lower IC levels at baseline. Conclusion: We confirm the feasibility of an RCT with patients randomized to CSMT or a wait list control group. There may be a subgroup of AS/LDH patients who will significantly benefit from high-velocity, lowamplitude CSMT. A large scale clinical trial is now underway to test these preliminary findings.

\section{9}

Beneficial effects of regular exercise prior to thoracolumbar spinal surgery. Kim Vu, ${ }^{1}$ Greg McIntosh ${ }^{2,3}$ Darren Roffey, ${ }^{1}$ Eve Tsai, ${ }^{1}$ Alexandra Stratton, ${ }^{1}$ Philippe Phan, ${ }^{1}$ Stephen Kingwell ${ }^{1}$ Eugene Wai, ${ }^{1}$ Michael Fobnson. ${ }^{4}$ From the ${ }^{1}$ The Ottawa Hospital, Ottawa, Ont.; ${ }^{2} \mathrm{CBI}$ Health Group, Toronto, Ont.; ${ }^{3}$ Canadian Spine Outcomes Research Network, Toronto, Ont.; ${ }^{4}$ University of Manitoba, Winnipeg, Man.

Background: "Prehabilitation" (or "prehab") has been promoted as a preoperative intervention purported to improve patient outcomes, especially in the context of arthroplasty surgery. However, there is currently little evidence to support this theory following spine surgery. Our objective was to determine if there is an association between self-reported preoperative exercise and postoperative outcomes after thoracolumbar spinal surgery. Methods: We performed a retrospective multivariable analysis of the Canadian Spine Outcomes and Research Network (CSORN) database. All thoracolumbar surgery patients with degenerative pathology only were identified. Fracture or tumour patients were excluded. Multivariable logistic regression was used to adjust for possible confounding effects of age, sex, number of operated levels, instrumentation, revision surgeries, current smoking status and Charlson comorbidity index. Patients who reported that they exercised regularly (i.e., at least twice per week) for at least 6 weeks before surgery were compared with those who did not report exercising regularly. Results: There were 1105 patients in the regular exercise group compared with 2788 in the nonexercising control group. There was no significant difference in improvement in the Oswestry Disability Index at 12 months' follow-up between the 2 groups. In univariate analyses, the regular exercise group trended toward having fewer adverse events $(5.5 \%$ vs. $7.3 \%, p=0.0532)$, and significantly shorter length of stay above expected $(0.3$ v. $1.0 \mathrm{~d}, p<0.0001)$. In multivariable analysis, the regular exercise group had fewer adverse events (adjusted odds ratio $[\mathrm{OR}]$ 0.695, 95\% confidence interval [CI]
0.51-0.95), and were less likely to have a length of stay greater than expected for their procedure (OR $0.690,95 \%$ CI $0.57-0.84$ ). Conclusion: Using a national Canadian database, after adjusting for confounding, we showed that patients who exercised regularly before surgery had significantly shorter length of stay and fewer postoperative adverse events. Further study is required to determine whether a "prehab" program is effective, although our results suggest that cost savings from reduced length of stay may justify the expense.

\section{5}

Sociodemographic characteristics of distinct patterns of low back pain. Greg McIntosh, Hamilton Hall, Thomas Carter. From the CBI Health Group, Toronto, Ont.

Background: Most previous research on patient characteristics of back pain classification has focused on clinical factors. This study presents new and original findings that concentrate on sociodemographic traits. The purpose of this study was to compare sociodemographic characteristics based on a syndrome or clinical pattern classification approach to low back pain (LBP). Methods: This is a retrospective review of prospectively collected LBP cases from 8 provinces. Patient enrolment occurred between January 2014 and June 2017. Baseline data were recorded at the initial assessment across a range of sociodemographic variables. All patients had mechanical LBP as determined by the Saskatchewan Spine Pathway triage methodology. Results: We identified 25313 patients as follows: 8535 patients with Pattern 1 PEP (prone extension positive, i.e., relieved with prone extension) (33.7\%); 12239 with Pattern 1 PEN (prone extension negative, i.e., not relieved with prone extension) (48.4\%); 3651 with Pattern 2 (relieved with flexion) (14.4\%); 55 with Pattern 3 (aggravated with flexion) (2.2\%); and 333 with Pattern 4 (relieved with flexion) $(1.3 \%)$. The mean age of the cohort was 42 years (SD $13.5)$, and $52 \%$ of patients were male; mean symptom duration was 64 (median 18) days. Multivariable logistic regression revealed different characteristics for each pattern. Patients with Pattern 1 PEP were more likely to be younger (odds ratio [OR] 0.994), male (OR 1.11), have high baseline functional status (OR 1.01 ), have no lawyer involvement (OR 1.32), show no sleep disturbance (OR 1.28), and continue to work (OR 1.15). Patients with Pattern 1 PEN were more likely to have lower baseline function (OR 0.99), lawyer involvement (OR 1.21) and sleep disturbance (OR 1.31), and were not likely to be working (OR 1.17). Patients with Pattern 2 were more likely to be female (OR 1.19), with high baseline function (OR 1.02) and no lawyer involvement (OR 1.36). Patients with Pattern 3 were more likely to be older (OR 1.02), to have lower baseline function (OR 0.94) and no lawyer involvement (OR 2.42), and to not use medication (OR 1.70). Patients with Pattern 4 were more likely to be older (OR 1.02) and male (OR 1.68), with lower baseline function (OR 0.965) and no lawyer involvement $(\mathrm{OR}=2.08)$, were more likely to smoke (OR 1.84), and more likely to be chronic (OR 1.83). Conclusion: These data further suggest that LBP is a heterogeneous entity that not only has distinct clinical markers, but sociodemographic characteristics; thus, a classification approach to LBP is warranted in both clinical and research settings. The results provide a unique contribution to the literature because little has been published on sociodemographic differences (similarities and differences) of low back pain subgroups. 


\section{8}

Improving postoperative patient-reported benefits and satisfaction after spinal fusion with a single preoperative education session. Donna Eastwood, ${ }^{1,2}$ Neil Manson, ${ }^{1,2,3,4}$ Richard Paixao, ${ }^{1,2}$ Erin Bigney, ${ }^{3}$ Kate Ellis, ${ }^{3}$ Eden Richardson, ${ }^{3}$ Edward Abrabam. ${ }^{1,2,3,4}$ From the ${ }^{1}$ Saint John Regional Hospital, Saint John, NB; ${ }^{2}$ Horizon Health Network, Saint John, NB; ${ }^{3}$ Canada East Spine Centre, Saint John, NB; ${ }^{4}$ Dalhousie University, Department of Surgery, Saint John, NB.

Background: The primary objective was to determine whether participation in a preoperative multidisciplinary educational session would result in improved postoperative patient satisfaction and reduce patient dissatisfaction with surgical expectations. A secondary objective investigated whether participation resulted in improvements in postsurgical pain and disability. Methods: A retrospective cohort study was conducted using Canadian Spine Outcomes and Research Network (CSORN) data. All participants were elective spinal fusion patients $(n=206)$. Cohort 1 included patients who opted to participate in a preoperative multidisciplinary education session 3-6 weeks before their surgery $(n=103)$. The single, 2-hour education session included interactive discussions with nursing staff, physiotherapy and occupational therapy, concentrating on what patients should expect and how to best prepare for surgery. Cohort 2 included patients who opted out of the educational session $(n=103)$. Outcome measures of interest were collected at 6-18 weeks postoperatively and included the Oswestry Disability Index (ODI), numerical rating scales for back and leg pain (NRS-B/L), CSORN questions pertaining to the patient's satisfaction with surgery and whether the surgery met a variety of patient expectations. Continuous variables were compared with analysis of variance, and categorical variables with $\chi^{2}$ analyses. Significance was set at $\alpha<0.05$. Results: Patients who took part in the presurgical education sessions were significantly more satisfied with their surgery compared with the control cohort $(p=0.014)$. Patients who did not participate in the education session failed to have their expectations met in terms of improvement in daily activities $(p=0.03)$, improvement in walking capacity $(p=0.03)$ and back pain reduction $(p=001)$. There was a statistically significant effect of participation in the educational session reducing postoperative back pain $(p=0.03)$, although this improvement did not reach clinically significant levels. Conclusion: Participation in a single 2-hour educational session before surgery positively impacted patient satisfaction, expectations and back pain. Managing patients' expectations before surgery is a viable tool for improving patient outcomes.

\section{3}

Prediction rule for determining discharge destination after posterior thoracolumbar spinal fusion procedures using the Canadian Spine Outcomes and Research Network (CSORN). Fan Fiang, ${ }^{1}$ Greg McIntosh, ${ }^{2}$ Mayilee Canizares, ${ }^{1}$ Michael Weber, ${ }^{3}$ Raja Rampersaud. ${ }^{1}$ From the ${ }^{1}$ University Health Network, University of Toronto, Toronto, Ont.; ${ }^{2}$ Canadian Spine Outcomes and Research Network, Toronto, Ont.; ${ }^{3}$ Department of Surgery, McGill University, Montreal, Que.

Background: Patients unable to be discharged home after spinal operations may require a prolonged stay in hospital while waiting for placement at rehabilitation facilities, thus creating reduced capacity within the health care system. Preoperative prediction of discharge destination could have significant impact on health system capacity and planning. Our objective was to create a prediction rule to determine the probability of being discharged home after thoracolumbar posterior spinal fusion. Methods: Using multicentre prospectively collected data from the CSORN database, a retrospective analysis was conducted of patients who underwent posterior thoracolumbar fusion between 2008 and 2017 ( $n=1988$ ). Multivariable logistic regression was used to identify predictors of patient discharge destinations - home $(n=$ $1807)$ or other facilities $(n=181)$ - using a backward selection procedure. A data-splitting technique was used to develop and test the multivariable models. Results: The mean age of the cohort was 59.9 years, with $52.6 \%$ women. Regression analyses identified 9 significant preoperative predictors of discharge home: age $<65$ years (odds ratio [OR] 2.349); not living alone (OR 2.48); lower Oswestry Disability Index (ODI) score (OR 0.96); diagnosis of deformity (OR 1.96) or spondylolisthesis (OR 2.1); American Society of Anesthesiologists (ASA) score other than III (OR 1.6); minimally invasive surgical (MIS) procedure (OR 2.8); levels of fusion < 3 (OR 4.4); and absence of preoperative neurologic deficit (OR 1.26). The final model was internally validated and confirmed the same predictors. Receiver operating characteristic curve analysis revealed area under the curve of 0.846 (sensitivity $=90 \%$, specificity $=45 \%$ ). A clinical prediction rule for the final model is represented by the following equation: $\log$ (odds of success $)=1.097-0.711($ age $<66)+0.797$ (living not alone $)-$ 0.029 (ODI score) +0.391 (diagnosis of deformity) +0.817 (diagnosis of spondyolisthesis) +0.367 (not ASA III) +1.40 (MIS procedure $)+1.41(<3$ levels $)+0.485$ (no neurologic deficit). Conclusion: Using the CSORN registry, we constructed a highly sensitive preoperative prediction rule to determine postoperative discharge destination following posterior thoracolumbar instrumented fusion. Further refinement of the prediction rule will allow the development of a simple point-based preoperative spine fusion discharge prediction tool for use by perioperative interprofessional surgical teams.

\section{1}

The impact of frailty and sarcopenia on postoperative outcomes in adult spine surgery: a systematic review of the literature. Eryck Moskven, ${ }^{1}$ Étienne Bourassa-Moreau, ${ }^{1}$ Rapbaële Charest-Morin, ${ }^{2}$ Alana M. Flexman, ${ }^{1}$ Fobn T. Street. ${ }^{1}$ From the ${ }^{1}$ University of British Columbia, Vancouver, BC; ${ }^{2}$ Centre Hospitalier Universitaire de Québec, Québec, Que.

Background: We sought to identify currently used measures of frailty and sarcopenia in the adult spine surgery literature, and to assess their ability to predict postoperative outcomes: death, morbidity, length of stay (LOS) and discharge disposition. In addition, we sought to determine which is the best clinical measure of frailty and sarcopenia for predicting outcome after spine surgery. Frailty and sarcopenia have been identified as predictors of death and adverse events (AEs) in numerous nonsurgical and nonspine populations. This is an emerging area of interest and study in patients undergoing spinal surgery. Methods: We performed a systematic literature review using the PRISMA methodology of Medline, PubMed, Ovid, Embase, and Cochrane databases from 
January 1950 to August 2017. All studies that examined measures of frailty or sarcopenia in adult patients who were undergoing any spinal surgery were included. The literature was synthesized, and recommendations were proposed based on the GRADE system. Results: The initial search yielded 53 results, 11 of which met our complete inclusion criteria: 7 reported on measures of frailty, and 4 reported on measures of sarcopenia. Frailty, assessed using a variety of measurement tools, was a consistent predictor of death, major and minor morbidity, prolonged hospital stay and discharge to a centre of higher care for adult patients undergoing spinal surgery. The relationship between sarcopenia and postoperative outcomes was inconsistent owing to the lack of consensus regarding definition, measurement tools and the wide variability in sarcopenia measured in the spinal population. Conclusion: Frailty is predictive of AEs, death, LOS and discharge disposition in a number of distinct spinal surgery populations. The impact of sarcopenia on postoperative outcomes is equivocal given the current state of the literature. The relationship between the spinal pathology, frailty, sarcopenia and how they interact to yield outcomes remains to be clarified. Frailty and sarcopenia are potentially useful tools for risk stratification of patients undergoing spinal surgery. This systematic review was registered with PROSPERO (registration no. 85096).

\section{9}

The need to improve prediction of responders and nonresponders in elective spine, hip and knee surgery: a healtheconomic argument. Y. Raja Rampersaud, Kala Sundararajan, Antbony V. Perruccio, Rajiv Gandbi, F. Rod Davey, Syed Khalid, Christian Veillette, Nizar N. Mabomed. From the University Health Network, University of Toronto, and the Krembil Research Institute, University Health Network, Arthritis Program, Toronto, Ont.

Background: An estimated $20 \%-25 \%$ of elective spine, hip and knee surgeries have poor outcomes, suggesting that considerable resources are being spent on elective procedures that do not benefit the patient. Our objective was to evaluate the cost utility of elective hip, knee and spine surgery on the basis of patient-reported surgical outcomes. Methods: Hospital costs were obtained for a cohort of elective surgeries at a Toronto tertiary-care centre in 2011/12 comprising inpatient and outpatient spine procedures in addition to hip and knee replacements. Patients completed the 12or 36-item short-form (SF12 or SF36) health survey before and 1 year after surgery. Patients were classified as responders if they achieved clinically important improvement 1 year postsurgery, defined as 4.6-point improvement on the SF-12/36 physical component summary score. One-year incremental cost-utility ratios (ICURs) were calculated as cost per quality-adjusted life year (QALY) gained after surgery, determined using utility scores from the short-form 6-dimension (SF-6D). Results: The sample comprised 48 inpatient spine, 39 outpatient spine, 150 hipreplacement, and 166 knee-replacement patients. The mean age of patients was 64 years, and $47 \%$ of patients were female. We deemed $71 \%$ of patients to be responders: $62 \%$ of spine, $85 \%$ of hip and $63 \%$ of knee patients. Mean hospital cost was $\$ 10937$ (2016 \$CAN, standard deviation [SD] \$6657), and patients gained a mean of 0.12 QALYs (SD 0.14) over the year after surgery, corresponding to an ICUR of \$89 464/QALY gained. Nonresponders had higher costs, a mean of $\$ 12447$ (v. $\$ 10319$ for responders, $p=$
$0.02)$, and smaller QALY gains by 1 year $(0.03$ v. 0.13 for responders, $p<0.01)$. The resulting overall 1 -year ICUR varied greatly with responder status: \$64 015/QALY in responders compared with \$460 399/QALY in nonresponders. Furthermore, ICUR ratios varied by operative site: responder/nonresponder ICURs were $\$ 115$ 694/\$1 574451 for inpatient spine; \$23 599/\$139 362 for outpatient spine; $\$ 57$ 975/\$118 068 for hip replacement; and \$77 761/\$1 121714 for knee replacement cases. Conclusion: Although elective hip or knee replacement and spine surgery have acceptable cost-effectiveness overall, they were grossly cost-ineffective in the $29 \%$ of patients who did not achieve clinically important change. These findings provide a strong economic case to support efforts to develop real-world predictive tools for these high-volume procedures.

\section{6}

Prognostic utility of pain diagrams for predicting surgical intervention among spine referrals. Khaled A. Almansoori, Michael M.H. Yang, Stephan Du Plessis. From the University of Calgary, Calgary, Alta.

Background: Pain diagrams (PDs) have been used as screening tools for the evaluation of back pain but show variability in their diagnostic and prognostic utility. Several studies have compared different PD methods, but none have investigated their role in predicting surgical intervention. We sought to evaluate 5 different PD interpretation methods: Uden, Ransford, Margolis, Ohnmeiss and the Quebec Task Force (QTF) approaches. Our primary objective was to compare the methods' likelihoods of predicting surgical intervention among patients. Methods: Prospective cohort design with consecutive patients who met our inclusion criteria. Patients were invited to complete a PD before their first encounter with their spine surgeon. Clinical management details were then retrospectively collected, and PDs were evaluated and graded by 2 surgeons to provide inter- and intra-rater reliabilities. Descriptive statistics, Spearman correlative analyses and binomial logistic regression analyses were performed using SPSS v.22 (SPSS Inc., Chicago, Illinois). Results: A total of 440 PDs were included in the study, with $22 \%$ of patients only reporting cervical spine symptoms and $11.6 \%$ of all participants ultimately undergoing surgical intervention. Binomial logistic regression showed weak relationships between PD interpretations and surgical intervention as shown by Nagelkerke variability estimations: Uden $\left(r^{2}=0.32\right)$, Ransford $\left(r^{2}=0.21\right)$, Margolis $\left(r^{2}=0.17\right)$, Ohnmeiss $\left(r^{2}=0.15\right)$ and the QTF methods $\left(r^{2}=0.31\right)$. Positive predictive value was highest among the Ohnmeiss method (20.3\%), and negative predictive value was highest with the QTF method $(95.97 \%)$. Inter-rater agreement was highest with the Ohnmeiss method (88.5\%) and lowest with the Margolis method (66.7\%). Application of PD assessments to the lumbar spine showed greater sensitivity and accuracy than did application to the cervical spine. Conclusion: Pain diagrams show good inter-rater agreement and generally provide negative prognostic value for determining which patients are unlikely to undergo surgical intervention. The most reliable pain diagram tools were the Ohnmeiss and QTF methods, whereas the Margolis method was the least reliable.

\section{0}

Preoperative psychological factors significantly add to the predictability of chronic narcotic use: a 2-year prospective 
study. Neil Manson, ${ }^{1,2,3}$ Kate Ellis, ${ }^{1}$ Erin Bigney, ${ }^{1}$ Eden Richardson, ${ }^{1}$ Dean Tripp, ${ }^{4}$ Edward Abrabam. ${ }^{1,2,3}$ From the ${ }^{1}$ Canada East Spine Centre, Saint John, NB; ${ }^{2}$ Horizon Health Network, Saint John Regional Hospital, Department of Orthopaedic Surgery, Saint John, NB; ${ }^{3}$ Dalhousie University, Faculty of Medicine, Department of Surgery, Saint John, NB; ${ }^{4}$ Departments of Psychology, Anesthesiology and Urology, Queen's University, Kingston, Ont.

Background: We wanted to determine whether psychological factors are significant predictors of narcotic use 2 years after thoracolumbar spine surgery. Methods: We conducted a prospective observational study that included 191 consecutively enrolled adult patients who had undergone thoracolumbar spine surgery. Patients who had undergone a previous spine surgery were excluded. Baseline measures of interest included the Pain Catastrophizing Scale (PCS), Tampa Scale for Kinesiophobia, Multidimensional Scale for Perceived Social Support (MSPSS), Chronic Pain Acceptance Questionnaire (CPAQ-8), Oswestry Disability Index (ODI), numeric rating scales for back and leg pain (NRS-B/NRS-L), short-form 12 (SF-12) Mental Component Summary (MCS), narcotic use and demographic variables. Data were collected using Canadian Spine Outcomes and Research Network (CSORN) questionnaires alongside validated psychologic scales. The measure of interest at 2 years after surgery was narcotic use. Narcotic use was collapsed into binary categories of use and no use. Descriptive statistics were run. We used $\chi^{2}$ analysis for categorical variables and an analysis of variance for continuous variables. Significant variables were built into a binary logistic regression to determine predictors of postoperative narcotic use. Significance was set at $\alpha<0.05$. Results: A total of $27.23 \%$ of the sample were using narcotics 2 years after surgery. The regression model included ODI, NRS-L, time with condition, chief complaint, preoperative drug use, sex, MCS, PCS subscale helplessness and CPAQ subscale pain willingness, and was significant $\left(\chi^{2}=[13, n=191] 54.99 ; p=0.000\right)$. The model accounted for $39.6 \%$ of the variance in narcotic use and correctly predicted in $79.7 \%$ of cases. Psychological variables accounted for $9.6 \%$ of the variance over and above the other predictors. Conclusion: Psychological factors in the preoperative period are significant predictors of narcotic use 2 years after surgery. The likelihood of chronic narcotic use after surgery is predictable and therefore may be proactively managed. Identifying predictors of chronic postsurgical narcotic use can enable surgeons to provide their patient with preventative resources. Managing chronic narcotic use is imperative, because it is central to the patient's overall health and quality of life.

\section{3}

Limitations of the National Surgery Quality Improvement Program in evaluation of risk for patients undergoing emergent surgery for spine metastases. Hanbing Zhou, Nicolas Dea, Charles Fisher, Tamir Ailon, Marcel Dvorak, Brian Kwon, Scott Paquette, Fobn Street. From the Division of Spine, Department of Orthopaedics, University Of British Columbia, Vancouver, Canada.

Background: Life expectancy and treatment options for metastatic spine disease continue to evolve. Prospective identification of complications is essential for risk stratification and treatment planning. The National Surgery Quality Improvement Program is currently popular, although its accuracy, validity and applicability to metastatic spine patients is unknown. We sought to examine acute care complications of surgery for spine metastases by comparing Spine Adverse Events Severity System (SAVES) and NSQIP, and to assess the NSQIP online tool predictive ability for adverse events in metastatic spine patients. Methods: All patients at our institution who underwent emergent surgery for spine metastases from 2012 to 2016 were identified. Adverse events were examined from both databases. Complication prevalence and relative risk were compared between NSQIP and SAVES. The NSQIP prediction tool was tested using primary and secondary tumour characteristics, hospital-stay characteristics, surgical invasiveness, patient demographics and patient comorbidities. Results: We identified 188 patients with complete data in both databases. Overall complication rates were $28 \%$ in NSQIP and $74.9 \%$ in SAVES. Adverse events more reliably identified in SAVES included wound complications (5.6 more; $p<0.05)$, delirium (2.8 more; $p<0.001)$, neuropathic pain $(3.2$ more; $p<0.001)$, urinary tract infection $(1.4 ; p<0.05)$, pressure sore $(2.9 ; p<0.001)$ and intraoperative adverse events $(2.3 ; p<0.05)$. Six adverse events more frequently identified by SAVES impacted length of stay $(p<$ $0.05)$. Risk factors such as tumour characteristics and severity of deficit more reliably correlated to adverse events collected through SAVES. Reoperation, in-hospital death and 30-day readmission rates were identical for both databases. The current NSQIP online tool had low predictive ability $(\mathrm{C}$-index $<0.6)$ for major complications. Conclusion: We found SAVES to be superior to NSQIP in identifying important medical and surgical complications in patients undergoing emergent surgery for metastatic disease of the spine. The current NSQIP online tool has low predictive ability for major complications.

4

Surgical site infection in spinal metastasis: incidence and risk factors. Amer Sebaaly, ${ }^{1}$ Zbi Wang, ${ }^{1}$, Francis Abed Rabbo, ${ }^{2}$ Ghassan Boubez, ${ }^{1}$ Daniel Shedid. ${ }^{2}$ From ${ }^{1}$ Department of Orthopedics, Centre Hospitalier de l'Université de Montréal,_Montreal, Que.; ${ }^{2}$ Department of Neurosurgery, Centre Hospitalier de l'Université de Montréal, Montreal, Que.

Background: Surgical site infection (SSI) of spinal metastasis surgery represents the most common postoperative surgical complication with high morbidity and mortality. The main objective of this paper is to evaluate the incidence of SSI in spinal metastasis surgery and its risk factors. Methods: Preoperative, operative and postoperative data were collected, as were modified Tokuhashi score and Frankel score at all time checkpoints. We divided SSI between superficial and deep SSI and between early (<90 d) and late SSI. Multiple logistic regression analysis was used to identify independent risk factors, with $\mathrm{p}<0.05$ as the significance threshold. Results: We included 376 patients with an incidence of SSI of 5.1\% (superficial SSI: 3.5\%; deep SSI: $1.6 \%$ ). Cervicothoracic surgery was associated with the highest incidence of SSI, whereas lumbar surgery had the lowest incidence. Smoking, higher number of spinal metastasis, higher body mass index (BMI), history of radiation therapy and higher American Society of Anesthesiologists (ASA) score were the preoperative 
factors associated with increased risk of SSI. Increased operative bleeding and increased number of fixed vertebra increased the SSI incidence, and SSI increased hospital stay by a mean of 22 days. When all variables were analyzed in a multiple regression model, only duration $\geq 4$ hours and ASA $\geq 3$ were independent risk factors for the occurrence of SSI. Conclusion: This is the largest series in the literature to report the incidence of SSI (5.1\%). Smoking, higher BMI, higher number of spinal metastasis, higher ASA score, preoperative radiation therapy, higher number of fused vertebrae, intraoperative bleeding $\geq 2000 \mathrm{~mL}$ and neurologic deterioration are risk factors for SSI occurrence. Only ASA $\geq 3$ and operative duration $\geq 4$ hours are independent risk factors for this complication. Finally, SSI is associated with increased hospital stay, increased 30-day mortality and decreased survival rates.

\section{4}

Adverse events profile in en bloc resection for metastasis and surgery for primary bone tumor. Shreya Srinivas, Raphael Charest Morin, Heidi Britton, Tamir Ailon, Charles Fisher, Mike Boyd, Marcel Dvorak, Scott Paquette, Brian Kwon, Fobn Street, Nicolas Dea. From the Vancouver Spine Surgery Insititute, Vancouver, BC

Background: The purpose of this study was to determine the adverse event (AE) profile in patients undergoing en bloc resection for spinal metastases or surgery for primary bone tumour of the spine. Methods: Patients who underwent surgery for metastases with a curative intent or surgical excision of a primary tumour of the spine between Jan. 1, 2009, and July 31, 2017, were included. Instances of AE were collected on a standardized form (Spine Adverse Events Severity System version 2 [SAVES V2]) at weekly dedicated morbidity and mortality rounds. Prospective data were collected on demographics, primary tumour histology, neurologic status, surgical intervention details, marginal status, Enneking appropriateness and all AEs (perioperative and postoperative). Results: There were 112 patients (64 women, 40 men, median age $51 \mathrm{yr}, 116$ procedures) included with primary bone tumour $(n=96)$ or metastatic spine lesion $(n=16)$. Surgical resection margin was Enneking appropriate (EA) in $73 \%$ and Enneking inappropriate (EI) in $27 \%$ of procedures for primary bone tumour. There was at least $1 \mathrm{AE}$ observed in $70.6 \%$ of patients, with 1 death. Intraoperative AEs occurred in $27.7 \%$ of patients, most commonly incidental durotomy (19.5\%), visceral or neurovascular injury (20.7\%) and massive blood loss (23\%). Postoperative AEs occurred in $65.5 \%$ of patients and were usually due to systemic infection $(39.5 \%)$ or a cardiac event $(35.5 \%)$. Incidence of thromboembolic events (deep vein thrombosis or pulmonary embolism) was $10.5 \%$. About $23.6 \%$ of patients experienced postoperative delirium, and neurologic deterioration was recorded in $14 \%$ of patients. Implant-related complications (malposition, failure) in the intraoperative and postoperative period was seen in $12 \%$ of procedures. Occurrence of AEs was marginally higher with EI (75\%) than with EA resection (66\%), but was similar regardless of tumour pathology (69\% in metastatic lesions and $71 \%$ for primary bone tumour). Wound-related complications $(22.4 \%)$ were significantly higher for procedures around fixed spine. Conclusion: En bloc resection for metastatic tumours and surgery for primary bone tumours are associated with high inci- dence of AEs. This should be of significant consideration in preoperative planning for patient counselling and to develop preventative strategies.

78

Development and validation of clinical prediction models of survival and clinical outcomes for patients with metastatic epidural spinal disease: a systematic review. Anick Nater, ${ }^{1}$ Fetan H. Badbiwala, ${ }^{1}$ Fames Hong, ${ }^{2}$ So Kato, ${ }^{1}$ Melanie Anderson, ${ }^{3}$ David Choi, ${ }^{4}$ Michael G. Fehlings. ${ }^{1,2,3,5}$ From the ${ }^{1}$ Toronto Western Hospital, Toronto, Ont.; ${ }^{2}$ Krembil Research Institute, Toronto, Ont.; ${ }^{3}$ University Health Network, Toronto, Ont.; ${ }^{4}$ University College London, London, United Kingdom; ${ }^{5}$ University of Toronto, Toronto, Ont.

Background: In multivariable prognostic research, the development and external validation are the first phases typically involved toward the establishment of clinical prediction models (CPMs) in practice. This systematic review aims to identify and assess CPMs created to predict clinical outcomes in patients with metastatic epidural spinal disease (MESD) and subsequent validation studies. Methods: Three electronic databases were searched (Jan. 1, 1990, to June 20, 2017), without language restriction, to identify studies that developed or evaluated CPMs predicting any clinical outcomes in adult patients with MESD (PROSPERO: CRD42017072908). Selected studies were then assessed based on their accordance with the Transparent Reporting of a multivariable prediction model for Individual Prognosis or Diagnosis (TRIPOD) statement. Results: Among 7275 unique full-text articles, 107 were included. Among the 43 articles describing the development of a CPM, 25 did not include any assessment related to internal validity/ model performance while 13 reported the number of outcome events and 6 how missing data were handled and 3 reported outcome predicted probabilities. We identified 80 studies evaluating CPMs. Among the 27 articles with the term "validation," "validated" or "validity" in the title or abstract, missing data for predictors and outcome, number of outcome events and both calibration and discrimination were specifically mentioned in 8 , 16 and 3 studies, respectively. Conclusion: Since 1990, over 40 CPMs predicting clinical outcomes in patients with MESD were developed and 80 studies performing some sort of evaluation of these CPMs were published. Based on the items included in the TRIPOD statement, the majority of these studies did not report on key methodological and data analysis elements. The lack of rigor in the development and validation of CPMs may explain why most CPMs are not generally used in clinical practice.

\section{8}

Evidence from the Epidemiology, Process and Outcomes of Spine Oncology (EPOSO) cohort: surgical versus radiation therapy for the treatment of cervical metastases. Michael Bond, ${ }^{1}$ Anne Versteeg, ${ }^{2}$ Arjun Sabgal, ${ }^{3}$ Peter Varga, ${ }^{4}$ Daniel Sciubba, ${ }^{5}$ James Schuster, ${ }^{6}$ Michael Weber, ${ }^{7}$ Michelle Clarke, ${ }^{8}$ Laurence Rbines, ${ }^{9}$ Stefano Boriani, ${ }^{10}$ Chetan Bettegowda, ${ }^{5}$ Michael G. Feblings, ${ }^{11}$ Paul Arnold, ${ }^{12}$ Ziya Gokaslan, ${ }^{13}$ Charles Fisher. ${ }^{1}$ From the ${ }^{1}$ Combined Neurosurgical and Orthopaedic Program, Vancouver General 
Hospital and University of British Columbia, Vancouver, BC; ${ }^{2}$ Department of Orthopedics, University Medical Center Utrect \& University Utrecht, Utrecht, Netherlands; ${ }^{3}$ Department of Radiation Oncology, Sunnybrook Odette Cancer Centre and University of Toronto, Toronto, Ont.; ${ }^{4}$ National Center for Spinal Disorders and Buda Health Center, Budapest, Hungary; ${ }^{5}$ Department of Neurosurgery, Johns Hopkins University School of Medicine, Baltimore, Md.; ' ${ }^{6}$ Department of Neurosurgery, Hospital of the University of Pennsylvania, Philadelphia, Pa.; ${ }^{7}$ Division of Surgery, McGill University and Montreal General Hospital, Montreal, Canada; ${ }^{8}$ Department of Neurosurgery, Mayo Clinic, Rochester, NY; ${ }^{9}$ Department of Neurosurgery, Division of Surgery, The University of Texas MD Anderson Cancer Center, Houston, Tex.; ${ }^{10}$ GSpine4 Spine Surgery Division, IRCCS Istituto Ortopedico Galeazzi, Milan, Italy; ${ }^{11}$ Division of Neurosurgery, University of Toronto and Toronto Western Hospital, Toronto, Ont.; ${ }^{12}$ Department of Neurosurgery, The University of Kansas Hospital, Kansas City, Kans.; ${ }^{13}$ Department of Neurosurgery, The Warren Alpert Medical School of Brown University and Rhode Island Hospital and The Miriam Hospital, Providence, RI.

Background: Cervical metastases have unique clinical considerations because of complex neighbouring anatomy, and the unique biomechanical regions within the cervical spine. The literature regarding cervical metastases is limited to retrospective studies without comparison of different treatment regimens. The purpose of this study was to compare surgery (with or without radiation) with radiation alone for the management of cervical metastases in a prospective manner. Methods: Patients who underwent surgery and/or radiotherapy for cervical metastases between August 2013 and February 2017 were identified from the Epidemiology, Process and Outcomes of Spine Oncology (EPOSO) observational cohort. Demographic, diagnostic, treatment and health-related quality of life (HRQOL) (numerical rating scale for pain (NRS pain), EurQOL 5-dimension [EQ-5D], 36-item short-form version 2 [SF-36v2] and Spine Oncology Study Group Outcomes Questionnaire [SOSGOQ]) measures were prospectively collected at baseline and at 6 weeks, 3 months and 6 months postintervention. Results: Fifty-five patients who received treatment for cervical metastases were identified: 38 underwent surgery (with or without radiation) and 17 received radiation alone. Surgically treated patients had higher spine instability neoplastic scores compared with the radiation-alone group (13.0 [standard deviation (SD) 2.8] v. 8.0 [SD 2.8], $p<0.001$ ) and were more likely to have mechanical neck pain $(89.5 \%$ v. $37.5 \%$, $p<0.001)$. Surgically treated patients presented with significantly higher NRS pain scores and lower HRQOL scores compared with the radiation-alone group $(p<0.05)$. From baseline to 6 months posttreatment, surgically treated patients showed significant improvements in NRS pain, EQ-5D and SOSGOQ version 2.0 scores compared with nonsignificant improvements in the radiotherapy-only group. Conclusion: Surgically treated patients presented with significantly worse baseline pain and HRQOL scores compared with patients who underwent radiotherapy alone. Preservation of pain and HRQOL was observed for patients treated with radiotherapy only compared with significant improvements for surgically treated patients.

\section{0}

Predictive factors of survival in a surgical series of metastatic epidural spinal cord compression and a complete external validation of eight multivariable scoring systems in a prospective North American multicentre study. Anick Nater, ${ }^{1}$ Lindsay A. Tetreault, ${ }^{2}$ Branko Kopjar, ${ }^{3}$ Paul Arnold, ${ }^{4}$ Mark Dekutoski, ${ }^{5}$ Joel Finkelstein, ${ }^{6}$ Charles Fisher, ${ }^{7}$ Jobn France, ${ }^{8}$ Ziya Gokaslan, ${ }^{9}$ Laurence Rbines, ${ }^{10}$ Peter Rose, ${ }^{11}$ Arjun Sabgal, ${ }^{6}$ James Schuster, ${ }^{12}$ Alexander Vaccaro, ${ }^{13}$ Michael G. Feblings. ${ }^{1}$ From the ${ }^{1}$ University of Toronto, Toronto, Ont.; ${ }^{2}$ University College Cork, Cork, Ireland; ${ }^{3}$ University of Washington, Seattle, United States; ${ }^{4}$ Kansas University Medical Center, Kansas City, Kans.; ${ }^{5}$ The Core Institute, Sun City West, Ariz.; ${ }^{6}$ Sunnybrook Health Sciences Centre, Toronto, Ont.; ${ }^{7}$ Vancouver General Hospital, Vancouver, BC; ${ }^{8}$ West Virginia University, Morgantown, WV; ${ }^{9}$ Rhode Island Hospital, Providence, RI; ${ }^{10}$ University of Texas, Houston, Tex.; ${ }^{11}$ Mayo Clinic, Rochester, NY; ${ }^{12}$ Hospital of the University of Pennsylvania, Philadelphia, Pa.; ${ }^{13}$ Thomas Jefferson University and Rothman Institute Orthopaedics, Philadelphia, Pa.

Background: This study was designed to identify preoperative predictors of survival in surgically treated patients with metastatic epidural spinal cord compression (MESCC), to examine how these predictors are related to 8 prognostic models, and to perform the first full external validation of these models in accordance with the Transparent Reporting of a Multivariable Prediction Model for Individual Prognosis or Diagnosis (TRIPOD) statement. Methods: One hundred forty-two surgically treated patients with MESCC were enrolled in a prospective, multicentre North American cohort study and were followed for 12 months or until death. Cox model was used. Noncollinear predictors with $<10 \%$ missing data, $\geq 10$ events per stratum and $p<0.05$ in univariable analysis were tested through a backward stepwise selection. For the original and revised Tokuhashi, Tomita, modified Bauer, van der Linden, Bartels, OSRI and Bollen, we examined calibration graphically, discrimination with Harrell c-statistics and survival stratified by risk groups with the Kaplan-Meier method and log-rank test. Results: The following were significant in univariable analysis: type of primary tumour, sex, organ metastasis, body mass index, preoperative radiotherapy to MESCC, 36-item short form version 2 (SF-36v2) physical component (PC) and EurQOL 5-dimension (EQ-5D). Breast, prostate and thyroid primary tumour (hazard ratio [HR] 2.9; $p=0.0005$ ), presence of organ metastasis (HR 2.0; $p=0.005)$ and SF-36v2 PC (HR 0.95; $p<$ $0.0001)$ were associated with survival in multivariable analysis. Predicted prognoses poorly matched observed values on calibration plots; Bartels' calibration slope was 0.45 . Bollen $(0.61 ; 95 \%$ confidence interval [CI] 0.58-0.64) and Bartels' (0.68; 95\% CI $0.65-0.71)$ had the lowest and highest c-statistics, respectively. Conclusion: The primary tumour type (breast, prostate, or thyroid), an absence of organ metastasis, and a lower degree of physical disability are preoperative predictors of longer survival for surgical MESCC patients. These results are in keeping with current models. This full external validation of 8 prognostic models of survival in surgical MESCC patients has revealed that calibration is poor, especially for long-term survivors, whereas discrimination is possibly helpful. 
5

Anterolateral cervical kyphoplasty for metastatic cervical spine lesions. Amer Sebaaly, ${ }^{1}$ Zhi Wang, ${ }^{1}$ Francis Abed Rabbo, ${ }^{2}$ Ghassan Boubez, ${ }^{1}$ Daniel Shedid. ${ }^{2}$ From the ${ }^{1}$ Department of Orthopedics, Centre Hospitalier de l'Université de Montréal, Montreal, Que.; ${ }^{2}$ Department of Neurosurgery, Centre Hospitalier de l'Université de Montréal, Montreal, Que.

Background: Even though the spine is the third most common site of metastasis, the cervical spine is affected the least, with an incidence of $10 \%-15 \%$. Surgical decompression is challenging because of the proximity of neural and vascular elements. Kyphoplasty for cervical spine metastasis was described in small case reports with promising results. Our objective was to evaluate the clinical and radiologic efficacy of anterolateral kyphoplasty for cervical spinal metastasis. Methods: We performed a retrospective analysis of a prospectively collected single-centre spine metastasis database for cervical kyphoplasty cases. Demographic data included age, sex and origin tumour diagnosis. Other variables included modified Tokuhashi score, spine instability neoplastic scores (SINS), preoperative visual analog score (VAS), analgesic medication of the patients and opioid use. Postoperative data included postoperative day 1 VAS, duration of hospital stay, self-reported functional outcome and last follow-up VAS. Results: We identified 11 patients with cervical spine metastases treated with 15 levels of kyphoplasty, with a mean age of 62.5 years. Mean Tokuhashi score was 8.1 and mean SINS was 7.85 . Mean preoperative pain was 7.1 and the mean number of preoperatively consumed analgesics was 1.8 , with $82 \%$ of patients using opioids. Total bleeding volume was $100 \mathrm{~mL}$. Mean complicationfree length of stay was 2.6 days with a decrease of postoperative pain (VAS $=2.8 ; p<0.05$ ). There was a $56 \%$ decrease of opioid dosage and the number of consumed analgesics $(1.09 ; p=0.004)$. A total of $82 \%$ of the patients reported excellent improvement on last follow-up self-assessment. Conclusion: This series represents the largest series of vertebral augmentation using balloon kyphoplasty for cervical spinal metastasis. This technique is associated with low postoperative complication, significant decrease in patient pain, lower use of opioids and shorter length of stay in hospital. The main indications for vertebral kyphoplasty are lytic lesions of the cervical spine, painful lesions refractive to medical treatment, SINS greater than 6 , but less than 10 , and modified Tokuhashi of less than 12 .

16

Characterizing the molecular landscape of sarcomatoid differentiation in prostate cancer bone metastasis. KarlPhilippe Guérard, ${ }^{1}$ Walead Ebrabimizadeb, ${ }^{1}$ Fadi Brimo, ${ }^{2}$ Michael Weber, ${ }^{3}$ Facques Lapointe. ${ }^{1}$ From the ${ }^{1}$ Research Institute of the McGill University Health Centre, Cancer Research Program, Department of Surgery, Division of Urology, Montreal, Que.; ${ }^{2}$ Research Institute of the McGill University Health Centre, Department of Pathology, Montreal, Que.; ${ }^{3}$ Research Institute of the McGill University Health Centre, Department of Surgery, Division of Orthopaedics, Montreal, Que.

Background: Advanced prostate cancer (PCa) often metastasizes to bones, leading to debilitating pain. Because bone metastases are rarely treated surgically, the limited access to tissue has hampered their genomic profiling. We have started collecting samples from spine metastasis surgeries for molecular profiling and recently identified a case of sarcomatoid differentiation. Mestastases are mostly acinar adenocarcinoma, but very rare cases show admixture of sarcomatoid differentiation, a highly aggressive histologic subtype. Whether the sarcomatoid clonally derives from the epithelial component is a matter of debate. We hypothesize that epithelial cells dedifferentiate into sarcomatoid cells and originate from the same malignant process. The overall objective of this study was to compare the molecular profiles of both subtypes by assessing genomic and proteomic alterations from the resected material on fresh and fixed samples and derived cell cultures. Methods: Surgical resection of PCa bone metastases was performed at the Montreal General Hospital. Blood was collected before surgery, and fresh material was either fresh-frozen; formalin-fixed, decalcified and paraffin-embedded (FFPE); or digested with collagenase for cell culture. Hematoxylin and eosin staining and immunohistochemistry with specific antibodies were performed on FFPE tissue. Cell cultures derived from the tumour were monitored and subjected to immunocytochemistry and immunofluorescence after fixation. Nucleic acids and proteins were extracted from the cultures. Results: Histopathologic assessment asserted sarcomatoid differentiation along adenocarcinoma in defined clusters. Immunostaining highlighted the existence of 2 compartments: adenocarcinoma cells being epithelial marker pan-cytokeratins (CK) positive $(+)$ and mesenchymal marker vimentin (Vim) negative (-), whereas surrounding sarcomatoid cells were CK- and Vim+. Some cell cultures were enriched for the same morphological subtypes, visually mirroring tissue display, and differentially expressed those markers. Specific genomic alterations identified in those cultures confirmed their malignant nature. Conclusion: We have identified markers that can, for this case, delineate both compartments in situ. RNA, DNA and protein analysis on tissue and matched cell cultures, from both components, will assess the molecular landscape of sarcomatoid differentiation. This work should provide molecular evidence of the origin of sarcomatoid differentiation, which might share characteristics with other lethal forms of PCa.

32

Ewing sarcoma of the spine: prognostic variables for survival and local control in surgically treated patients. Raphaële Charest-Morin, ${ }^{1}$ Michael S. Dirks, ${ }^{2}$ Sbreyaskumar Patel, ${ }^{3}$ Stefano Boriani, ${ }^{4}$ Alessandro Luzzati, ${ }^{4}$ Michael G. Feblings, ${ }^{5}$ Charles G. Fisher, ${ }^{6}$ Mark B. Dekutoski, ${ }^{7}$ Richard Williams, ${ }^{8}$ Nasir A. Quraishi, ${ }^{9}$ Chetan Bettegowda, ${ }^{10}$ Ziya L. Gokaslan, ${ }^{11}$ Niccole M. Germscheid, ${ }^{12}$ Peter Pal Varga, ${ }^{13}$ Laurence D. Rbines. ${ }^{3}$ From the ${ }^{1}$ Centre Hospitalier Universitaire de Québec, Québec, Que.; ${ }^{2}$ Walter Reed National Military Medical Center, Bethesda, Md.; ${ }^{3}$ The University of Texas MD Anderson Cancer Center, Houston, Tex.; ${ }^{4}$ IRCCS Istituto Orthopedico Galeazzi, Milan, Italy; ${ }^{5}$ University of Toronto and Toronto Western Hospital, Toronto, Ont.; ${ }^{6}$ University of British Columbia and Vancouver General Hospital, Vancouver, BC; ${ }^{7}$ The CORE Institute, Sun City West, Ariz.; ${ }^{8}$ Princess Alexandra Hospital, Brisbane, Australia; ${ }^{9}$ Nottingham University Hospital NHS Trust, Nottingham, United Kingdom; ${ }^{10} \mathrm{Johns}$ Hopkins University School of Medicine, Baltimore, Md.; ${ }^{11}$ The 
Warren Alpert Medical School of Brown University, Providence, RI; ${ }^{12}$ Research Department, AOSpine International, Davos, Switzerland; ${ }^{13}$ National Center for Spinal Disorders and Buda Health Center, Budapest, Hungary.

Background: Treatment of primary Ewing sarcoma (ES) of the spine is complex. Ambiguity remains regarding the role and optimal type of surgery in the treatment of spinal ES. The objectives of this study were to quantify mortality and local recurrence after surgical treatment of spinal ES and to determine whether an Enneking appropriate procedure and surgical margins (en bloc resection with wide or marginal margins) are associated with improved prognosis. Methods: The AOSpine Knowledge Forum Tumor developed a multicentre database including demographics, diagnosis, treatment, mortality and recurrence rate data for spinal ES. Patients were stratified based on surgical margins and Enneking appropriateness. Survival and recurrence were analyzed using Kaplan-Meier curves and log-rank tests. Results: Fiftyeight patients diagnosed with primary spinal ES underwent surgery. Enneking appropriateness of surgery was known for 55 patients; 24 (44\%) were treated Enneking appropriately (EA) and $31(56 \%)$ were treated Enneking inappropriately (EI). A statistically significant difference in favour of EA-treated patients was found with regards to survival $(p=0.034)$. Neoadjuvant and postoperative chemotherapy was significantly associated with increased survival $(p=0.008)$. Local recurrence occurred in $22 \%$ $(n=5)$ of patients with an EA procedure compared with $38 \%(n=$ 11) of patients with an EI procedure. The timing of chemotherapy treatment was significantly different between the Enneking cohorts $(p<0.001)$, and all EA-treated patients received chemotherapy treatment. Local recurrence was not significantly different between Enneking cohorts ( $p=0.140$ ), but intralesional surgical margins and a previous spine tumour operation were associated with increased local recurrence $(p=0.025$ and $p=$ 0.018 , respectively). Conclusion: Surgery should be undertaken when an en bloc resection with wide or marginal margins is feasible. An EA surgery correlates with improved survival, but the impact of other prognostic factors needs to be evaluated. En bloc resection with wide or marginal margins is associated with local control.

\section{7}

Nanoparticle-based targeted zoledronate delivery to treat bone metastasis secondary to prostate cancer. Elie Akoury, ${ }^{1}$ Bardia Barimani, ${ }^{1}$ Pouyan Abangar, ${ }^{1}$ Karl-Pbilippe Guerard, ${ }^{2}$ Facques Lapointe, ${ }^{2}$ Lisbet Haglund, ${ }^{1}$ Derek Rosenzweig, ${ }^{1}$ Michael Weber. ${ }^{1}$ From ${ }^{1}$ Research Institute of the McGill University Health Centre, Injury Repair Recovery Program, Department of Surgery, Division of Orthopaedics, Montreal, Que.; ${ }^{2}$ Research Institute of the McGill University Health Centre, Cancer Research Program, Department of Surgery, Division of Urology, Montreal, Que.

Background: Zoledronate ( $\mathrm{Zol})$ is a bisphosphonate (BP) class drug used in treatment of spinal metastasis. Systemic BP treatment is the current standard for delivering medication to patients. However, BP can cause multiple adverse effects. Interestingly, our group has shown that local Zol delivery blocks tumour-induced osteolysis in mice while preventing the occurrence of adverse effects associated with systemic administration.
Over the past few decades, nanoparticles have been explored as an exciting method for delivering anticancer drugs directly to tumour sites. These nanoparticles allow for high and local administration of drugs while avoiding the complications of systemic delivery. Our aim is to develop a valuable tool that delivers Zol locally for the treatment of bone metastasis secondary to prostate cancer. Methods: To test the Zol-releasing nanobead system, fluorescent Zol was incubated overnight with mesoporous nanoparticles that were either uncoated or coated with a thin chitosan shell. The nanoparticles were dispersed in aqueous media. Aliquots from the dispersed solution were taken daily for up to 14 days, and fluorescent Zol was measured using a plate reader. To test the effect of Zol on the prostate cancer cell line LAPC4, cells were seeded, incubated with non-fluorescent Zol for 7 days and assessed for proliferation rate and metabolic activity using the Vybrant MTT cell proliferation and alamarBlue assays, respectively. Results: Chitosan-coated nanoparticles hold and release more Zol over time in aqueous media compared with uncoated nanoparticles. In addition, treating LAPC4 with Zol results in significantly reduced proliferation and metabolic activity. Conclusion: We will test the proliferation and metabolic activity of patient-derived bone metastasis cells after Zol treatment. In addition, we will test the Zol-releasing nanobead system on LAPC4 and patient-derived cells. Zol-releasing nanoparticles could constitute a therapeutic promise to combat metastatic spine tumours secondary to prostate cancer. These nanoparticles can be integrated into commercial bone putty to develop a bioactive bone graft after bone tumour resection to deliver localized BP drug that facilitates bone stability and healing while preventing tumour recurrence.

\section{5}

Lumbar surgery in octagenarians. Really? Felipe Nares, ${ }^{1}$ Greg McIntosh, ${ }^{2}$ Alexandra Soroceanu, ${ }^{3}$ Kennetb Thomas. ${ }^{3}$ From the ${ }^{1}$ University of Calgary, Calgary, Alta.; ${ }^{2}$ Canadian Spine Outcomes and Research Network, Toronto, Ont.; ${ }^{3}$ Foothills Medical Center, University of Calgary, Calgary, Alta.

Background: Lumbar decompression for spinal stenosis is the most common spine surgical procedure performed. With our aging population, elective spine surgery is becoming more common in older adults. The aim of this study was to identify whether age, specifically being more than 80 years old, influences the effects of lumbar decompression surgery on leg pain at 1-year follow-up. Methods: Prospectively collected Canadian Spine Outcomes and Research Network (CSORN) data was used to identify surgical patients with a pathologic diagnosis of lumbar stenosis. The cohort was divided into 2 groups $(<80 \mathrm{yr}, \geq 8 \mathrm{yr})$. The impact of age on 1-year improvement in health-related quality of life (HRQL) was assessed using analysis of covariance, adjusting for significant confounders identified on univariate analysis. Results: One-year HRQL data were available for 1507 patients (<80 yr, $n=1414$; $\geq 80 \mathrm{yr}, n=93)$. Median patient age was 61 (range 18-90) years, and $49 \%$ of patients were female. Adjusting for confounders showed that older patients were more likely to have a higher American Society of Anesthesiologists (ASA) score, less likely to smoke, less likely to be receiving insurance payments, less preoperative narcotic use and lower body mas index. The 2 groups were similar in sex distribution, exercise status, preoperative neurologic deficit, symptom duration, number 
who had a fusion and previous lumbar surgery. After adjusting for confounders, there was no statistically significant difference in the 1-year improvement on the Oswestry Disability Index (-18.90 v. $-21.27, p<0.798$ ), EurQOL 5-dimension (EQ5D; -0.06 v. 0.01, $p<0.072$ ), short-form 12 mental component (SF-12 MCS; 0.60 v. $3.54, p<0.067)$, numerical rating scale (NRS) back pain $(-3.88$ v. $-3.45, p<0.16)$ and NRS leg pain $(-4.31 \mathrm{v} .-3.69, p<0.28)$. The $\geq 80$ years age group experienced statistically less improvement on SF-12 physical component (PCS; 5.96 v. 10.78, $p<$ 0.027 ) at 1 year compared with their younger counterparts (although the difference was smaller than the minimum clinically important difference for PCS). Conclusion: At 1-year postoperative follow-up, change in VAS leg pain and SF-12 PCS score showed significantly less improvement in patients aged 80 years and older, although these observed differences were not clinically significant. The remaining patient-reported outcomes appeared similar between the older and younger patient groups. When considering patient-reported outcomes, lumbar surgery in patients 80 years of age and older may be a reasonable choice.

\section{8}

Clinical outcomes research in lumbar spine surgery: Are 2-year follow-ups necessary? Oliver Ayling, Charles Fisher, Hanbing Zhou, Tamir Ailon, Greg McIntosh, Nicolas Dea. From the University of British Columbia, Vancouver, BC.

Background: There has been generic dictum in spine and musculoskeletal clinical research that 2-year follow-up is necessary for patient-reported outcomes (PROs); however, the rationale for 2-year follow-up is not evidence-based. The purpose of this study was to determine the PRO follow-up time necessary to ensure the effectiveness of a lumbar surgical intervention is appropriately evaluated. Methods: Using PROs from the Canadian Spine Outcomes and Research Network (CSORN) prospective database, the time-course to plateaued recovery after lumbar spine surgery was assessed for lumbar disc herniation, degenerative spondylolisthesis and spinal stenosis. One-way analyses of variance (ANOVAs) with post hoc testing were used to analyze PROs at baseline and at 3,12, and 24 months postoperatively on the following standardized PROs: EurQOL 5-dimension (EQ5D), Oswestry Disability Index (ODI), visual analogue scale (VAS) leg and back, and short-form 12 (SF-12) mental and physical scales. Results: There were significant differences determined by 1-way ANOVAs for all spine pathologies and specific PROs $(p<$ $0.0001)$. Time to plateaued recovery after surgery for lumbar disc herniation, lumbar spondylolithesis or lumbar stenosis followed the same time-courses for the following PROs: VAS back and leg, 3 months; ODI, 12 months; SF-12 physical, 12 months; and SF-12 mental, 3 months. Conclusion: Individual PROs after surgery for lumbar spine pathologies follow specific time-courses to plateaued recovery, suggesting that a 2 -year follow-up is not required for all outcomes to be accurately assessed. Ultimately, the clinical research question should dictate follow-up time and the outcome measure used; however, there is now evidence to guide the specific duration of follow-up for each PRO.

\section{0}

Residents' and fellows' participation and postoperative technical surgical outcomes in elective anterior cervical spine surgery. Mabmoud Bedaiwy, Eugene Wai, Philippe
Phan, Stephen Kingwell, Yabya Alqabtani, Mobammad Alswat, Abdulkarim AlRabie. From the Ottawa Spine Collaborative Analytics Network (OSCAN) and The Ottawa Hospital, Ottawa, Ont.

Background: The purpose of our study is to identify whether trainee participation in elective anterior cervical spine surgery cases is associated with deleterious technical outcomes. Methods: We performed a retrospective study using data from the National Surgical Quality Improvement Program database (NSQIP) for 2011 to 2012. We identified 5648 Anterior cervical decompression and fusions (ACDFs). Our inclusion criteria were ACDF as principal procedure performed as an elective procedure availability of information on trainee participation and outcomes. Our exclusion criteria included nonclean wound class and cancer or emergency surgery. Complications related to technical surgical issues including surgical site infection, wound dehiscence and unplanned return to the operating room were prospectively collected. Potential confounding factors considered included age, sex, American Society of Anesthesiologists (ASA) classification, outpatient operating room (OR), in addition to preoperative blood work, smoking, alcohol use, preoperative chronic diseases and operative diagnoses. Results: After applying inclusion and exclusion criteria, we identified 1920 patients available for analysis. Multivariate logistic regression analysis, with adjustments for confounding factors, showed that Resident or Fellow participation or year of training was not significantly related to surgeryrelated complications $(p>0.45)$. Multivariate regression analysis, with adjustments for confounding factors, showed that Resident or Fellow was significantly related to longer operative time (adjusted mean $58.7 \mathrm{~min}$ ) in OR $(p<0.0001)$. Conclusion: Using a large prospective multicentre North American database, we confirmed the anecdotal observations that operative time was significantly longer with trainee involvement. However, it is reassuring that the trainee participation did not result in any effect on surgical complication rates.

\section{0}

Canadian spine fellowship training: self assessment. Foel Finkelstein. From the Sunnybrook Health Sciences Centre, Toronto, Ont.

Background: A survey of recent spine fellowship graduates was used to assess the perceptions of their training with respect to appropriate emphasis and their satisfaction on decompression techniques compared with instrumentation. Methods: Graduated spine fellows and recently appointed staff were asked to answer questions of relative time spent during their fellowship on formal teaching of basic decompression techniques, specific techniques for difficult cases and direct instruction for instrumentation. The confidence level was also assessed for their ability to do a difficult decompression and to perform instrumentation. Finally, the trainees perception of their expectation to get a dural tear while performing a difficult decompression and their belief on the effect on outcome were determined. Results: There were 24 respondents, $80 \%$ of whom were orthopedic trained, and $37 \%$ of whom were currently in their fellowship year. Up to 3 mentors were rated with time spent devoted to specific teaching for decompression and for instrumentation. The results indicated there was variability in the total teaching time between mentors. 
Direct technical teaching for instrumentation was felt to be substantial in $90 \%$ of respondents; for decompression, $65 \%$ of respondents felt there was substantial direct decompression teaching. The confidence level for instrumentation was rated as very confident in $66 \%$ of respondents and $50 \%$ for performing a difficult decompression. The wish to have more training in decompressions was $58 \%$ compared with $42 \%$ for instrumentation. Fifty-eight percent of respondents expected to get a dural tear while decompressing a severe stenosis, and $42 \%$ believed this had little effect if any on outcome. Conclusion: The self assessment of spinal surgery training in Canada was measured in this survey with regard to the confidence one has in a difficult decompression and for instrumentation of the spine. There was a desire to obtain more direct technical training in decompressions, although there was less perceived need for the same for instrumentations. Several factors may create the opportunity for more experience and training for the instrumentation of the spine. The perceptions about dural tears reinforce the need for more direct training in performing decompressions. The survey is intended to open a country-wide dialogue on our emphasis on spine surgery training.

\section{1}

Trainee perspectives on procedural competencies during spine surgery clinical fellowship. Antony Bateman, ${ }^{1,2}$ Feremie Larouche, ${ }^{1}$ Chrisitina Goldstein ${ }^{3}$ Daniel Sciubba, ${ }^{4}$ Theodore Choma, ${ }^{3}$ Brandon Lawrence, ${ }^{5}$ Joseph Cheng, ${ }^{6}$ Michael G. Feblings, ${ }^{7}$ Scott Paquette, ${ }^{8}$ Albert Yee ${ }^{1,7}$ From the ${ }^{1}$ Sunnybrook Health Sciences Centre, Toronto, Ont.; ${ }^{2}$ Royal Derby Hospital, Derby, United Kingdom; ${ }^{3}$ University of Missouri, Columbia, Mo.; ${ }^{4}$ Johns Hopkins Hospital, Baltimore, United States; ${ }^{5}$ University of Utah, Salt Lake City, Utah; ${ }^{6}$ Yale University, New Haven, Conn.; ${ }^{7}$ University of Toronto, Toronto, Ont.; ${ }^{8}$ University of British Columbia, Vancouver, BC.

Background: It remains important to align competence-based objectives for training deemed important by fellowship educators to those that are desired by their trainees. We know that after residency training, spine surgeons often complete fellowshiplevel training before entering independent practice. Although residency programs follow an established syllabus, only some fellowship training programs are accredited. The Canadian Spine Society recently established a syllabus of competency-based educational objectives for fellowship training that includes specific procedural competencies. The primary aim of this study was to determine trainee views on the relative importance of these specific procedural competencies. Secondarily, we aimed to evaluate self-perceived confidence in procedural performance at the commencement and completion of fellowship. Methods: Questionnaires were administered to 68 clinical fellows enrolled in the AOSpine North America (AOSNA) fellowship program during the 2015/16 academic year. A Likert scale was used to quantify trainee perspectives on the relative importance of specific procedural competencies to their training base on an established curriculum including 53 general and 22 focused or advanced procedural competencies. We measured trainee self-perceived confidence in performing procedures at the commencement and completion of their program. Statistical analysis was performed on fellow demographic data and procedural responses. Results:
Our response rate was $82 \%(56 / 68)$ for the initial survey and $69 \%$ (47/68) for the follow-up survey. Most syllabus procedures were regarded as of high importance, with some differences observed on comparing neurosurgical and orthopedic trainees. There were more variable replies to some focused or advanced competencies (e.g., spinal injections). We identified several procedures of high importance and low confidence among fellows (i.e., upper cervical, thoracic discectomy surgery), which highlights an educational opportunity. Overall procedural confidence increased from 4.2 (standard deviation [SD] 1.3) in the initial survey to 5.4 (SD 0.8) in follow-up survey $(p<0.0001)$. Conclusion: Understanding trainee goals for clinical fellowship remains important, and this study advances knowledge on the importance of specific procedural competencies. Identification of areas of low procedural confidence and high importance to training will better guide fellowship programs and supervisors in the strategic delivery of the educational experience.

\section{2}

Surgical feasibility and results of oblique lateral interbody fusion at L5-S1 level. Alisson R. Teles, W. Bradley Jacobs, Fred H. Nicholls, Peter Lewkonia, Roger Cho, Kenneth C. Thomas, Ganesh Swamy. From the Spine Program, University of Calgary, Calgary, Alta.

Background: Oblique lateral interbody fusion at L5-S1 level (OLIF51) is a recently introduced less invasive technique that presents several clear advantages over open surgical approaches for this level. The literature lacks information on appropriate patient selection criteria for OLIF51 and predictors of successful execution. Our objective was to identify predictors for feasibility of OLIF51. Methods: We performed a retrospective review of consecutive cases of OLIF51 done between 2014 and 2017. Failure was defined as a major vascular injury or abortion of the procedure owing to difficulty of vessel mobilization or complete exposure of the disc space. Potential predictors for failure were age, sex, surgical indication, previous abdominal surgery, position of the iliocaval junction (ICJ), position of the left common iliac vein (LCIV), absence of fat behind the LCIV, flattening of the LCIV and presence of a large anterolateral osteophyte at L5-S1. Anatomical features were assessed using preoperative magnetic resonance imaging (MRI). Using SPSS, bivariate and multiple regression analyses were performed to identify predictors for infeasibility of OLIF51. Intra- and interobserver reliability of the measurements were assessed among 7 fellowship-trained spine surgeons and 3 spine fellows. Results: A total of 62 OLIF51 procedures were attempted during the study period, of which $53(87.1 \%)$ were completed and 8 $(12.9 \%)$ were aborted intraoperatively. The rate of vascular injury was $6.5 \%(n=4)$. In the bivariate analyses, the risk factors for infeasibility of OLIF51 were position of the LCIV $(p<$ $0.0001)$, low ICJ $(p=0.001)$, absence of fat behind the LCIV $(p=0.018)$, and male sex $(p=0.024)$. Logistic regression demonstrated that medial position of the LCIV (adjusted odds ratio [OR] 26.29, $p=0.001$ ) and male sex (adjusted OR = 9.68, $p=$ 0.019 ) were independent predictors for infeasibility of OLIF51. Median distance of LCIV from midline was $11.6 \mathrm{~mm}$ (25th percentile $=7 \mathrm{~mm}, 75$ th percentile $=17 \mathrm{~mm}$ ) for successful cases and $-4 \mathrm{~mm}$ (25th percentile $=-6.5 \mathrm{~mm}, 75$ th percentile $=$ $1.5 \mathrm{~mm})$ for failed cases $(p=0.002)$. Conclusion: Careful 
preoperative anatomical assessment of the LCIV on MRI may improve patient selection for this novel technique.

\section{8}

Long-term practice trends of fellowship-trained spine surgeons in Canada. Khaled A. Almansoori, ${ }^{1}$ Zenon Zirko, ${ }^{2}$ Dale Williams. ${ }^{2}$ From the ${ }^{1}$ Sheikh Khalifa Medical City, Abu Dhabi, United Arab Emirates; ${ }^{2}$ McMaster University, Hamilton, Ont.

Background: Spine surgeons have one of the highest fellowship participation rates among the medical subspecialties, with more than $88 \%$ of Canadian graduates completing at least one fellowship and $63 \%$ undertaking a second. Several studies have shown that a large proportion of surgeons narrow and limit their scope of practice within a decade of their fellowships. As a result, this study investigates the long-term practice trends of fellowshiptrained spine surgeons in Canada with the hypothesis that, over time, most surgeons limit their scope and volume of surgical practice. Methods: Cross-sectional self-reported semistructured surveys were distributed to 102 practising spine surgeons in Canada. Demographic data, fellowship education, clinical scope and details, practice motivations and employment information were collected to determine attrition rates, work environments and subspecialty practice trends and changes. Statistical analyses were performed using SPSS version 22 (IBM Corp., Chicago, Illinois). Results: The response rate was $35 \%$ $(n=35)$, with a mean age of 49 years and 16 years in practice; $94 \%$ of respondents were men, and $89 \%$ worked in an urban setting. Spine-related work comprised $72 \%$ of their practice, with orthopedic trauma and pediatrics being the most common complementary practices. About $66 \%$ of participants narrowed their scope of practice over time, with the most prognostic factors being rural work setting $\left(r^{2}=0.99\right)$ and age $\left(r^{2}=0.49\right)$. Practice dissatisfaction (31\%), followed by inadequate resources (28\%) and work volume or hours $(20 \%)$ were the most commonly reported reasons for limiting their practices, while physical disability and financial remuneration were the least commonly reported reasons. More than $74 \%$ of participants agreed that most of the skills they currently employ were acquired during their fellowship training. Overall, satisfaction rates were generally high, with rural spine surgeons expressing the highest proportion of dissatisfaction owing to limited resources and excessive working hours or volume. Conclusion: Most orthopedic spine surgeons continue to perform procedures they acquired during their fellowship training and generally narrow their scope of practice in relation to age and rural work settings. The most commonly cited reasons for restricting their practices were work satisfaction, followed by inadequate resources and work volume.

\section{5}

Predictive model for return to work after lumbar spine surgery in Canada. Alisson R. Teles, ${ }^{1}$ Steven Casba, ${ }^{1} \mathrm{~W}$. Bradley Facobs, ${ }^{1}$ Kenneth C. Thomas, ${ }^{1}$ Greg McIntosh ${ }^{2}$ Raphaele Charest-Morin, ${ }^{3}$ Jeff D. Golan, ${ }^{4}$ Carlo Santaguida, ${ }^{4}$ Peter Farzem, ${ }^{4}$ Jean A. Ouellet, ${ }^{4}$ Michael H. Weber. ${ }^{4}$ From the 'Spine Program, University of Calgary, Calgary, Alta.; ${ }^{2}$ CBI Health Group, Toronto, Ont.; ${ }^{3}$ Department of Orthopedic Surgery, Centre Hospitalier Universitaire de
Québec, Québec, Que.; ${ }^{4}$ McGill Scoliosis \& Spine Group, Montreal, Que.

Background: Work absenteeism after spine surgery is a significant contributor to financial and societal burden of spinal disorders. Identifying predictors for return to work (RTW) will help surgeons have a substantive personalized discussion with patients and promote realistic expectations before surgery. Our objective was to create a predictive model for RTW after elective lumbar spine surgery in Canada. Methods: Data from a prospective multicentre registry (Canadian Spine Outcome and Research Network [CSORN]) were used to assess RTW after lumbar spine surgery. Inclusion criteria were: being employed at the time of enrollment; age between 20 and 65 years; and diagnosis of lumbar disc herniation, stenosis, spondylolisthesis or disc degeneration. We assessed RTW using survival analysis, calculated by the method of Cox proportional hazards regression to find the best multivariable model predicting outcome using a backward selection procedure. The model performance was measured using a data-splitting technique in which an $80 \%$ random sample of the full data set was used for model development and the entire data set for validation. Results: A total of 1076 patients were included in the study. The median time to RTW was 58 (range 0-571) days (mean $82.5 \pm 76.1 \mathrm{~d}$ ). After adjusting for all other covariates in the model, shorter times to RTW were associated with patients who did not have workers' compensation or insurance claims (hazard ratio [HR] 1.43), were male (HR 1.55), had light (HR 1.33) or sedentary (HR 1.50) lifting requirements at work, had no need for help at work (HR 1.15), had symptoms lasting less than 1 year (HR 1.24), did not have fusion (HR 1.62), had a single-level operation (HR 1.38), had lower depression scores (HR 0.97) and were not from New Brunswick (HR 1.46) or Quebec (HR 1.45). The model presented adequate internal validity in the validation sample. Conclusion: We present a novel predictive model for RTW after lumbar spine surgery in Canada. Canadian spine care providers can use this model to educate patients and encourage them in shared decision-making regarding RTW after lumbar spine surgery.

67

Effect of surgical decompression on back pain in lumbar spinal stenosis: a Canadian Spine Outcome and Research Network registry study. Shreya Srinivas, ${ }^{1}$ Greg McIntosh, ${ }^{2}$ Charles Fisher, ${ }^{1}$ Nicolas Dea. ${ }^{1}$ From the ${ }^{1}$ Vancouver Spine Surgery Institute, Vancouver, BC; ${ }^{2}$ Canadian Spine Outcome Research Network, Toronto, Ont.

Background: Surgical decompression is usually offered to improve neurogenic claudication in patients with lumbar canal stenosis. These patients often have associated low back pain (LBP), and little is known about surgical results for this disabling symptom. The goal of the present study was to specifically quantify improvement of LBP following surgical decompression for lumbar canal stenosis and to identify factors associated with change in LBP in this population. Methods: Consecutive patients who underwent surgical treatment for lumbar spinal stenosis without instability (1-2 level) were prospectively enrolled in the multicentre Canadian Spine Outcomes Research Network (CSORN) registry. Patient-reported outcomes were collected at baseline and at 3,12 and 24 months after surgery. Our primary 
outcomes were change in LBP numeric rating scale (NRS) and Oswestry Disability Index (ODI). Multivariable logistic regression was used to model the relationship between the outcomes and potential factors associated with achieving minimal clinical important difference (MCID) in back pain using a backward selection procedure. Results: There were 1340 patients included in the analysis. The mean age was 65 years, and $59 \%$ of patients were male. Follow-up evaluations were available for 952 patients at 12 months and 548 patients at 24 months. Low back pain significantly improved 3 months after surgery and was sustained at 24 months $(p<0.001)$. We found that $74 \%$ of patients reached MCID in regards to NRS. Factors associated with sustained improvement (12 and 24 months) in LBP after surgical intervention were absence of narcotic usage, severity of LBP before surgery (high NRS) and being a nonsmoker. Conclusion: Low back pain was statistically and clinically significantly alleviated at 3 months after decompression surgery for lumbar spinal stenosis, and this improvement was maintained at 12 and 24 months' follow-up in most patients. Back pain severity, smoking status and narcotic usage were associated with LBP improvement.

\section{9}

Increased prevalence of chronic disease in back pain patients living in a car-dependent neighbourhood. Amy Zeglinski-Spinney, ${ }^{1}$ Darren Roffey, ${ }^{1,2}$ Stephen Kingwell, ${ }^{1,2,3,4}$ Philippe Phan, ${ }^{1,2,3,4}$ Eugene Wai, ${ }^{1,2,3,4}$ Denise Wai. ${ }^{1}$ From the ${ }^{1}$ Ottawa Spine Collaborative Analytics Network (OSCAN), Ottawa, Ont.; ${ }^{2}$ Ottawa Hospital Research Institute, Ottawa, Ont.; ${ }^{3}$ Division of Orthopaedic Surgery, The Ottawa Hospital, Ottawa, Ont.; ${ }^{4}$ Faculty of Medicine, University of Ottawa, Ottawa, Ont.

Background: Chronic diseases, including chronic back pain, result in substantial patient morbidity and societal burden. Improvement in physical fitness is strongly recommended for treatment and prevention. Walking is an easy modality to perform to achieve initial gains. However, most population studies rely on patient self-reported levels of activity, which are often inaccurate. Our objective was to determine if neighbourhood walkability, acting as a surrogate measure of physical fitness, was associated with presence of chronic disease in chronic back pain patients. Methods: We performed a retrospective, crosssectional study of prospectively collected data from 162 adult patients referred for assessment of their chronic back pain. Charlson Comorbidity Index (CCI) was calculated from data captured through a patient-completed survey and medical records review. Using patient postal codes, neighbourhood walkability was determined courtesy of Walk Score, a large-scale, public access walkability index that assigns a numerical walkability score to any address in Canada. The Walk Score algorithm awards points based on distance to closest amenities, yielding a score from 0 to 100 (0-50 points, car-dependent; 50-100 points, walkable). Multivariate analysis was used to adjust for confounding. Results: Based on their Walk Score, 69 patients (42.6\%) lived in a cardependent neighbourhood, while 93 (57.4\%) lived in a walkable neighbourhood. In unadjusted univariate analysis, the odds of having a chronic disease was 2.2 (95\% confidence interval [CI] 1.1-4.5) times greater for patients living in a car-dependent neighbourhood, and there was a significant trend $(p=0.0114$, Mantzel Hansel $c^{2}=6.4$ ) toward more severe CCI scores. A mul- tivariate logistic regression model, adjusting for age, sex, body mass index, smoking, median household income and percentage employment, showed an adjusted odds of 2.8 (95\% CI 1.1-4.5, $p=0.0110)$ times greater risk for having a chronic disease for patients living in a car-dependent neighbourhood. Conclusion: Our study shows a correlation between living in a car-dependent neighbourhood and presence of chronic disease. These results suggest that advocating for improved neighbourhood planning to permit greater walkability may help offset the burden of chronic disease.

76

Effect of the duration of symptoms on surgical outcomes in lumbar stenosis and disc herniation: a Canadian Spine Outcomes and Research Network registry study. Duncan Cushnie, Kennetb Thomas, Steven Casha. From the University of Calgary and Foothills Medical Centre, Calgary, Alta.

Background: Lumbar spinal stenosis and disc herniation are the most common spine pathologies requiring surgical intervention. A longer duration of neurologic compression could possibly lead to irreversible neural injury resulting in a failure of surgery to alleviate symptoms. We sought to determine if longer symptom duration in these conditions was associated with inferior surgical outcomes. Methods: The Canadian Spine Outcomes and Research Network (CSORN) registry was queried for all lumbar stenosis and disc herniation cases with neurogenic claudication or radiculopathy treated surgically. Spinal deformity and previous surgery were exclusion criteria. Cases were grouped based on symptom duration. Primary outcomes were change in disability and leg pain as measured by Oswestry Disability Index (ODI) and numerical rating scale (NRS) leg pain at 1-year postoperative follow-up. Results: We identified 482 lumbar stenosis and 293 lumbar disc herniation cases. Improvement in ODI was significantly correlated with shorter symptom duration in those patients with stenosis $(p=0.002)$, but not disc herniation $(p=0.08)$. Fewer lumbar stenosis patients with more than 1 year of symptoms achieved a minimal clinically important difference (MCID) in ODI $(67.8 \%)$ compared with those with less than 1 year of symptoms $(80.3 \% ; p=0.008)$. Mean NRS leg pain improvement also correlated with shorter duration of symptoms in lumbar stenosis $(p=0.03)$ but not lumbar disc herniation $(p=0.36)$. Conclusion: Greater duration of preoperative symptoms was associated with smaller improvements in leg pain and disability in lumbar stenosis patients after decompressive surgery.

\section{9}

Resolution and factors associated with low back pain after lumbar discectomy: a Canadian Spine Outcomes and Research Network (CSORN) registry study. Christian Iorio-Morin, ${ }^{1}$ Charles Fisher, ${ }^{2}$ Greg McIntosh, ${ }^{3}$ Nicolas Dea, ${ }^{2}$ CSORN Investigators. From the ${ }^{1}$ Université de Sherbrooke, Sherbrooke, Que.; ${ }^{2}$ University of British Columbia, Vancouver, BC; ${ }^{3}$ CSORN, Toronto, Ont.

Background: Lumbar discectomy is frequently performed to alleviate radicular pain resulting from disc herniation. Although this goal is typically achieved in most patients, additional improvement in low back pain has been reported inconsistently. The goal of this study was to better characterize how low back 
pain resolves following discectomy and identify factors associated with back pain improvement. Methods: We performed a retrospective analysis of prospectively collected data from the Canadian Spine Outcomes Research Network (CSORN) registry. Any patient who underwent discectomy (using any technique) for single-level lumbar disc herniation was eligible for inclusion. The primary outcomes were back pain numerical rating scale (BPNRS) and Oswestry Disability Index (ODI) assessed at 3, 12 and 24 months. Multivariable logistic regression was used to model the relationship between the outcomes and potential factors associated with a minimal clinically important difference (MCID) change in back pain, using a backward selection procedure. Results: There were 1479 patients included in the analysis; $54 \%$ were male, $56 \%$ were married and $46 \%$ were smokers. The chief complaint was radiculopathy (83\%); $48.5 \%$ underwent a minimally invasive procedure. Both BPNRS and ODI showed significant reduction at all time points postoperatively, by $39 \%-$ $52.7 \%(p<0.001)$, with $70 \%$ of patients achieving the MCID. In the various multivariate models, the factors most consistently associated with a clinically significant reduction in BPNRS and ODI were the preoperative BPNRS and ODI. Other significant factors included not having worker compensation or insurance claims, being younger and having shorter symptom duration (< 1 yr). Conclusion: In an unselected, real-life cohort, clinically significant improvement in low back pain was seen in $70 \%$ of patients after lumbar discectomy and was maintained up to 2 years. The level of preoperative pain is most associated with showing clinically significant improvement.

\section{1}

Patient expectations in spine surgery: a national Canadian perspective. Mayilee Canizares, ${ }^{1}$ Andrew Glennie, ${ }^{2}$ Ken Thomas, ${ }^{3}$ Christopher Bailey, ${ }^{4}$ Sean Christie, ${ }^{2}$ Eugene Wai, ${ }^{5}$ Charles Fisher, ${ }^{6}$ Antbony Perruccio, ${ }^{1}$ Y. Raja Rampersaud. ${ }^{1}$ From the ${ }^{1}$ Krembil Research Institute, University Health Network, Arthritis Program, Toronto, Ont.; ${ }^{2}$ QEII Health Sciences Centre, Halifax, NS; ${ }^{3}$ Foothills Medical Centre, Calgary, Alta.; ${ }^{4}$ Western University, London, Ont.; ${ }^{5}$ The Ottawa Hospital - Civic Campus, Ottawa, Ont.; ${ }^{6}$ Vancouver General Hospital, Vancouver, BC.

Background: Few studies have examined patients' expectations in spine surgery. The objectives of this study were to examine the most important change patients expected from their spine surgery and to examine the influence of sociodemographic and clinical characteristics on expected changes. Methods: Data from 4408 patients with a primary degenerative spinal diagnosis were found in the Canadian Spine Outcomes and Research Network (CSORN) registry and analyzed. Patients indicated on a questionnaire their level of expectation as it related to 7 separate dimensions as: no change (0), somewhat better (1), better (2) and much better (3). An overall expectations score (normalized to 100) was calculated, with higher scores reflecting greater surgical expectations. Patients also indicated which of the 7 areas was most important to them. Sociodemographic and clinical variables, including pain and disability from the Oswestry Disability Index (ODI) and the Neck Disability Index (NDI) for patients with thoracolumbar and cervical diagnosis, respectively, were also collected. Multiple linear regression was used to examine the adjusted associations between these variables and expectation scores. Results: Of the included patients, $28.9 \%$ ranked "improve leg/arm pain," 25.9\% ranked "back/neck pain," $21.0 \%$ ranked "general physical capacity" and $15.8 \%$ ranked "independence in everyday activities" as the most important expected change. No differences were found in the ranking of expectations by demographic or clinical variables. The mean expectation score was $55.0( \pm 29.9)$. Factors associated with higher expectations were: older age ( $\beta=1.6$ per $10-y r$ increment, $p<0.001)$, being in the labour force but not working compared with not in the labour force $(\beta=3.8, p=0.019)$, nonobese compared with obese $(\beta=2.6$, $p=0.011)$, fewer comorbidities $(\beta=-1.9, p<0.001)$, thoracolumbar site $(\beta=4.9, p=0.008)$, diagnosis of disc herniation $(\beta=5.5$, $p \leq 0.001$ or spondylolisthesis $(\beta=2.7, p=0.042)$ compared with stenosis alone, depressed $\operatorname{mood}(\beta=5.4, p<0.001)$, worse pain $(\beta=2.3, p<0.001)$ and worse disability $(\beta=0.12, p=0.003)$. Conclusion: The most important expectation of spine surgery is highly variable across patients. Identifying specific individual expectations is important for improved shared decision-making and presurgery education, and may be key to understanding patient satisfaction following surgery.

\section{2}

Patients' expectation fulfillment and satisfaction with spine surgery. Mayilee Canizares, ${ }^{1}$ Andrew Glennie, ${ }^{2}$ Ken Thomas, ${ }^{3}$ Christopher Bailey, ${ }^{4}$ Sean Christie, ${ }^{2}$ Eugene Wai, ${ }^{5}$ Charles Fisher, ${ }^{6}$ Anthony Perruccio, ${ }^{1}$ Y. Raja Rampersaud. ${ }^{1}$ From the ${ }^{1}$ Krembil Research Institute, University Health Network, Arthritis Program, Toronto, Ont.; ${ }^{2}$ QEII Health Sciences Centre, Halifax, NS; ${ }^{3}$ Foothills Medical Centre, Calgary, Alta.; ${ }^{4}$ Western University, London, Ont.; ${ }^{5}$ The Ottawa Hospital - Civic Campus, Ottawa, Ont.; ${ }^{6}$ Vancouver General Hospital, Vancouver, BC.

Background: The purpose of this study was to determine the extent to which patient expectations of spine surgery were fulfilled and to examine the association between unmet expectations and postoperative satisfaction. Methods: Canadian Spine Outcomes and Research Network (CSORN) data from 1985 patients with a primary degenerative spinal diagnosis and 1 year of followup data were used for analysis. Preoperative information regarding each patient's most important dimension (of 6) for which they were expecting change was collected. For each of these dimensions, patients reported whether the surgery fulfilled their expectations at 1-year follow-up. Logistic regression models were fit to examine the association between expectations being met and baseline sociodemographic (e.g., age, sex) and clinical variables (e.g., principal diagnosis), in addition to pre- and post-surgical changes in pain and disability scores. Results: Overall, $88.8 \%$ of patients reported that their most important expected change was met: $95.8 \%$ among those expecting improvement in independence in everyday activities; $94.5 \%$ for improvements in sporting activities/recreation; $89.0 \%$ and $87.3 \%$ for improvements in leg/ arm pain and back/neck pain, respectively; $85.4 \%$ for improvements in general physical capacity; and $82.7 \%$ of those expecting improvements in mental well-being. Factors associated with expectations being met were spondylolisthesis diagnosis versus stenosis and improvements in pre- to post-surgical pain and disability scores $(p<0.0001$ for all variables). In addition, about $93 \%$ of patients who had their expectations met were extremely or somewhat satisfied with their surgery, compared with $52.2 \%$ of 
patients with unmet expectations. Likewise, $4.2 \%$ of those with met expectations were extremely or somewhat dissatisfied, compared with $27.3 \%$ of those with unmet expectations. Similar trends were seen for each of the expectation dimensions examined. Conclusion: Most patients reported their most important surgical expectation as having been met; 1 in 10 patients reported unmet expectations. Although met or unmet expectations were strongly associated with satisfaction or dissatisfaction, these results must be considered against a backdrop of "most important expected change" being highly variable across patients. Preoperative inquiry and education regarding realistic individual patient expectations are paramount to patient satisfaction with spine surgery.

43

Opioid use in elective spine surgery: predictors of preoperative use and provincial variations across Canada. JeanChristophe Murray, ${ }^{1}$ Mayilee Canizares, ${ }^{2}$ 7. Denise Power, ${ }^{2}$ Antbony V. Perruccio, ${ }^{2,3,4}$ Y. Raja Rampersaud. ${ }^{1,2,5}$ From the ${ }^{1}$ Division of Orthopaedic Surgery, Department of Surgery, University of Toronto, Toronto, Ont.; ${ }^{2}$ Arthritis Program, Krembil Research Institute, Toronto, Ont.; ${ }^{3}$ Healthcare \& Outcomes Research, Krembil Research Institute, Toronto, Ont.; ${ }^{4}$ Institute of Health Policy, Management \& Evaluation, Dalla Lana School of Public Health, University of Toronto, Toronto, Ont.; ${ }^{5}$ Divisions of Neurosurgery, Department of Surgery, University of Toronto, Toronto, Ont.

Background: Opioid use is an emergent public health issue in Canada, and use among surgical spine patients is common. However, large studies to assess patient factors associated with preoperative opioid use are lacking. Our primary objective was to identify predictors of preoperative opioid use among Canadian elective spine surgical patients. Secondarily, we wanted to assess whether there was significant provincial variation in such usage. Methods: We performed a retrospective review of Canadian Spine Outcomes Research Network (CSORN) data across 8 provinces. Patients $(n=4375)$ with a primary degenerative spinal diagnosis who provided data on preoperative opioid use were included. The primary outcome was preoperative opioid status (daily, intermittent, no use). Multinomial logistic regression models were estimated to examine the associations of sociodemographic (e.g., age, sex, education) and clinical variables (e.g., diagnosis type, type of surgery) with preoperative opioid use. Proportions using opioids across provinces were compared. Results: Daily, intermittent and no preoperative opioid use were reported by $18.3 \%, 32.4 \%$ and $50.7 \%$ of patients, respectively. No significant difference was found in opioid use across provinces $(p=0.08)$. Independent factors significantly associated with preoperative daily opioid use were thoracolumbar diagnosis (odds ratio [OR] 2.73), depressed mood (OR 2.22), 3 or more comorbidities (OR 1.93), disc herniation diagnosis (v. stenosis) (OR 1.79), positive legal claim (OR 1.56), in labour force but not working (OR 1.54), anxious mood (OR 1.48), degenerative disc disease (OR 1.41) and being a current smoker (OR 1.37). Older patients, patients who exercised regularly and patients who were currently working were significantly less likely to report daily opioid use. With the exception of smoking, degenerative disc disease, work status and exercise, the same factors (with same direction of effect) were also associated with intermittent opioid use. Conclusion: Preoperative opioid use is highly prevalent in patients undergoing elective spine surgery across Canada, with $50 \%$ reporting intermittent or daily use. This large study lays the foundation for subsequent work to examine the impact of opioid use on health services and patient-reported surgical outcomes, and to identify potentially at-risk subpopulations of patients using opioids.

62

Opioid use in low back pain: patient characteristics, outcomes and costs. Eric 7. Crawford, ${ }^{1,2}$ Robert A. Ravinsky, ${ }^{1,3}$ Peter C. Coyte, ${ }^{2}$ Y. Raja Rampersaud. ${ }^{1,3}$ From the ${ }^{1}$ Division of Orthopaedic Surgery, University of Toronto, Toronto, Ont.; ${ }^{2}$ Institute of Health Policy, Management and Evaluation, University of Toronto, Toronto, Ont.; ${ }^{3}$ Division of Orthopaedic Surgery, Toronto Western Hospital, University Health Network, Toronto, Ont.

Background: Low back pain (LBP) is common among adults and is associated with enormous health care and societal costs. Opioids are the most commonly prescribed drug class for LBP, yet little evidence exists regarding their efficacy in this population or their health economic impact. The purpose of this investigation was to determine the treatment outcomes and costs of care associated with opioid use in adults with LBP. Methods: A retrospective review of the Inter-professional Spine Assessment and Education Clinics (ISAEC) research database containing prospectively collected outcomes was undertaken to identify adults with LBP without leg-dominant symptoms. Opioid-use, health economic data and outcomes were determined based on patient-reported data at entry to an LBP management program and at a 6-month follow-up. Outcomes were assessed with the Oswestry Disability Index (ODI). Health economic analyses were performed from a societal perspective and included health care use and work absenteeism data. Results: Of the patients meeting our inclusion criteria, $15.5 \%(183 / 1183)$ reported opioid use. A logistic regression model identified that opioid use was associated with smoking (odds ratio [OR] 1.82, 95\% confidence interval [CI] 1.12-3.02; $p=0.016$ ), a previous history of LBP (OR 1.94, 95\% CI $1.21-$ $3.11 ; p=0.006)$ and increased disability/ODI scores at baseline (opioid users: mean $=42.3$, standard deviation $[\mathrm{SD}]=16.4$; nonopioid users: mean $=29.7, \mathrm{SD}=15.9 ; p=0.025)$. Although patients with opioid use experienced equivocal reductions in disability at follow-up, they continued to have increased ODI scores relative to patients who did not report opioid use. Health economic analyses indicated that opioid use was associated with increased direct health care costs (opioid users: mean $=\$ 485.7$, SD 67.8; non-opioid users: mean $=\$ 298.3, \mathrm{SD}=21.6 ; p=$ 0.002 ) and a trend toward more days off work (opioid users: mean $=8.6, \mathrm{SD}=3.8$; non-opioid users: mean $=4.2, \mathrm{SD}=0.8$; $p=0.07)$. Conclusion: This investigation found that opioid use was associated with another addictive behaviour (smoking), recurrent episodes of LBP and increased disability at baseline and follow-up. Furthermore, opioid use did not improve outcomes and was associated with increased health care costs and possibly work absenteeism. Given these results, and the dangers associated with this drug class, opioid use is not recommended and should be minimized in adults with LBP. 
136

The influence of preoperative opioid use on self-reported pain and disability 1 year after elective spine surgery. FeanChristophe Murray, ${ }^{1}$ Mayilee Canizares, ${ }^{2}$ 7. Denise Power, ${ }^{2}$ Antbony V. Perruccio, ${ }^{2,3,4}$ Y. Raja Rampersaud. ${ }^{1,2,5}$ From the ${ }^{1}$ Division of Orthopaedic Surgery, Department of Surgery, University of Toronto, Toronto, Ont.; ${ }^{2}$ Arthritis Program, Krembil Research Institute, Toronto, Ont.; ${ }^{3}$ Healthcare \& Outcomes Research, Krembil Research Institute, Toronto, Ont.; ${ }^{4}$ Institute of Health Policy, Management \& Evaluation, Dalla Lana School of Public Health, University of Toronto, Toronto, Ont.; ${ }^{5}$ Divisions of Neurosurgery, Department of Surgery, University of Toronto, Toronto, Ont.

Background: Limited studies have demonstrated an association of preoperative opioid use with negative surgical outcomes. Our objective was to assess the influence of preoperative opioid use on patient-reported pain and disability 1 year after elective spine surgery. Methods: We performed a retrospective review of Canadian Spine Outcomes Research Network (CSORN) data across 8 provinces. Patients with a primary degenerative spinal diagnosis, preoperative opioid use data, and baseline and 1-year postsurgical patient-reported outcomes were included. Two outcomes at 1 year after surgery were examined: pain from a visual analog scale (0, no pain; 10 , unbearable pain) and disability from the Oswestry Disability Index (ODI) and the Neck Disability Index (NDI), for patients with thoracolumbar and cervical diagnoses, respectively. The main covariate of interest was preoperative opioid use (daily, intermittent, and no use). Other covariates included sociodemographic (e.g., age, sex, education, work status), lifestyle (e.g., smoking, body mass index), and clinical variables (e.g., depressive mood, surgery type). Multiple linear regression models were used to examine the associations between preoperative opioid use and 1-year postsurgical pain and disability, after adjusting for baseline levels of these variables, as well as the other study covariates. Results: In multivariable linear regression models, preoperative daily opioid use was independently associated with worse self-reported pain $(\beta=0.48 ; p=$ $0.003)$ and disability $(\beta=4.83 ; p<0.0001)$ at 1 -year postsurgery as compared with patients not using opioids. Intermittent use was associated with worse disability $(\beta=2.44 ; p<0.042)$ but not pain. Legal claims $(\beta=0.55 ; p=0.001)$, smoking $(\beta=0.47 ; p=0.007)$, anxiety $(\beta=0.85 ; p=0.004)$, and higher baseline pain $(\beta=0.24$; $p<0.0001)$ were associated with worse pain scores at 1 year. Worse disability scores were associated with older age $(\beta=0.90$; $p=0.029)$, legal claims $(\beta=5.93 ; p<0.0001)$, smoking $(\beta=2.83$; $p=0.013), 3$ or more comorbidities $(\beta=4.85 ; p=0.003)$, anxiety $(\beta=2.28 ; p<0.026)$, and higher baseline ODI/NDI $(\beta=0.44 ; p<$ $0.0001)$. Conclusion: Preoperative use of opioids in elective spinal surgery patients is independently associated with greater pain and disability at 1 year postsurgery. Future work will focus on examining efficacy of opioids and predictors of changes in opioid use postsurgery and its associated impact on outcomes.

\section{0}

Low back pain prognostic factors for time receiving wagereplacement benefits. Greg McIntosh, ${ }^{1}$ Hamilton Hall, ${ }^{1}$ Ivan Steenstra, ${ }^{2}$ Cyntbia Chen, ${ }^{3}$ Sheilab Hogg-Fobnson, ${ }^{3}$ Ben Amick, ${ }^{3}$ Teresa D'Elia, ${ }^{3}$ Thomas Carter. ${ }^{1}$ From the ${ }^{1} \mathrm{CBI}$
Health Group, Toronto, Ont.; ${ }^{2}$ Morneau Shepell, Toronto, Canada; ${ }^{3}$ Institute for Work \& Health, Toronto, Ont.

Background: Recent systematic reviews have identified only a few back pain studies that use "time-to-event" data. This study addresses that oversight with a unique and original data analysis approach using survival analysis, calculated using Cox regression. The objective was to develop a prognostic model that predicts time receiving workers' compensation benefits for low back pain claimants enrolled in any 1 of 20 postinjury rehabilitation clinics across Ontario. Methods: A retrospective review of prospectively collected data was used to identify an inception cohort. To obtain the outcome and predictor variables, patient data from 2 separate resources were linked: a clinical (physiotherapy) and an administrative (Ontario Workers' Compensation) database. Those with back pain claims filed between 2011 and 2015 were included and followed for 1 year from the date of accident $(n=1652)$. The independent variables assessed for predictive value were a back pain questionnaire, as well as clinical, demographic, and administrative factors. The outcome variable was cumulative number of calendar days receiving wage-replacement benefits. Results: Multivariable Cox proportional hazards regression revealed 8 significant predictors of shorter time on benefits: symptom duration less than 31 days (hazard rate [HR] 0.879); attending early intervention rehabilitation (HR 1.51); not working in the construction industry (HR 1.89); high Low Back Outcome Score (higher perceived function) (HR 1.025); younger age (HR 0.992); higher wage-replacement benefit rate (HR 1.00); intermittent (not constant) pain (HR 1.15); and no sleep disturbance (HR 1.15). A predictive score was calculated to categorize claimants as at high or low risk for chronicity; $23 \%$ were found to be at high risk. Conclusion: Building a predictive model should focus on the identification of key variables that can be easily identified and reliably collected in a clinical setting. Clinicians need to be aware of what factors differentiate people who become chronically disabled from those who do not. This study identifies 8 significant factors associated with shorter time on wage-replacement benefits. This multivariable model indicates a robust and reasonably accurate clinical prediction capacity.

59

Narcotic use trends in elective thoracolumbar spinal surgery patients. Alexandra Stratton, ${ }^{1}$ Greg McIntosh, ${ }^{2}$ Eugene Wai, ${ }^{1}$ Phillippe Phan, ${ }^{1}$ Stephen Kingwell, ${ }^{1}$ Darren Roffey. ${ }^{1}$ From the ${ }^{1}$ University of Ottawa, Ottawa, Ont., ${ }^{2}$ Canadian Spine Outcomes Research Network.

Background: Narcotic use has increased rapidly in the United States in recent years. There is an association between preoperative narcotic use and inadequate perioperative pain control, increased length of stay and poor spinal surgery outcomes. Our aim was to investigate patterns of narcotic use in Canadian spinal surgery patients, examine trends over time, and determine the effect of spinal surgery on postoperative narcotic use. Methods: Retrospective analysis of prospectively collected data on elective thoracolumbar surgery patients in the Canadian Spine Outcomes and Research Network (CSORN) database. Self-reported narcotic use at baseline, presurgery and 1 year postsurgery were compared. Baseline narcotic use by age, sex and presenting complaint were also compared. All patients meeting eligibility criteria 
from database inception to 2017 were included. Results: We identified 3511 patients who met the inclusion criteria. Over time (before 2014 to 2017), there was no significant change in the proportion of patients taking narcotics on a daily (range $32.3 \%-$ $38.2 \%$ ) or intermittent (range $13.7 \%-22.5 \%$ ) basis. There was no difference in the frequency of narcotic use at baseline and presurgery in patients who waited longer than 6 weeks for surgery. There were significantly more patients using narcotics with a chief complaint of back pain or radiculopathy than neurogenic claudication $(p<0.001)$, and who were younger $(<65$ years) than older $(p<0.001)$. At 1 year postsurgery, daily narcotic use decreased significantly from baseline $(34.5 \%-16.9 \%, p<0.01)$. Conclusion: Narcotic use in spinal surgery patients in Canada is widespread. Although narcotic use might decrease postoperatively, as surgeons, we are having little to no impact on our patients' narcotic use while on the wait list for surgery. An opportunity may exist to intervene in this critical preoperative stage to optimize surgical outcomes. Continued efforts to decrease narcotic use should be focused on patients less than 65 years old with radiculopathy or back pain.

\section{4}

Addition of dexmedetomidine and aprepitant to a multimodal postoperative pain regimen reduces narcotic requirements, emesis, and length of stay in treating adolescent idiopathic scoliosis. Andrew Tice, Heather Kent, Dong-Phuong Tran, Chan-Hee Jo, Brandon Ramo. From the Texas Scottish Rite Hospital, Dallas, Tex.

Background: We sought to assess the affect of institutional practice change in perioperative management of adolescent idiopathic scoliosis to optimize pain control while reducing adverse effects. The original protocol included an epidural catheter with infusion of hydromorphone and ropivicaine (group 1). The first alteration was to eliminate the hydromorphone from the epidural and augment with a dexmedetomidine intravenous infusion (group 2). The second alteration was addition of antiemetic aprepitant (group 3). All patients had supplemental oral narcotics, oral or intravenous acetaminophen, oral ibuprofen, intravenous ketorolac, and supplemental antiemetics. Methods: We performed a quality improvement project consisting of a retrospective review of patients undergoing posterior spinal fusion for adolescent idiopathic scoliosis over a 2-year period. The primary outcome was total analgesic use, further divided into narcotic and non-narcotic use. All doses of analgesics were converted, using equianalgesia calculations, to reference doses of oral morphine. Secondary outcomes include pain scores, episodes of nausea, emesis or pruritis, hours to ambulation, length of stay and adverse events. Results: We identified 171 eligible patients: 51 in group 1, 42 in group 2 and 78 in group 3 . Total analgesic requirements were significantly less in groups 2 and 3 compared with group 1, most likely explained by significantly reduced total narcotic requirement in groups 2 and 3 . We found no significant differences in pain scores when comparing maximum scores or standard deviation by postoperative day. Group 3 had significantly less nausea and emesis than either other group. Groups 2 and 3 had significantly fewer episodes of pruritis. Group 3 had the shortest length of stay, showing statistical significance on comparison with group 1. There were no differences detected with regards to nursing calls for hypotension, hours to ambulation, or hours to first bowel movement. Conclusion: The elimination of a narcotic epidural infusion and addition of dexmedetomidine significantly reduces postoperative narcotic use. Furthermore, addition of aprepitant significantly reduces episodes of nausea and emesis. Culmination of both these benefits resulted in a statistically significant, though clinically modest, reduction in length of stay.

\section{0}

The discrepancy between clinical measures and selfreported ratings of health status after surgery for degenerative cervical myelopathy: an introduction to response shift. Lindsay A. Tetreault, ${ }^{1,2}$ Mary Zbu, ${ }^{3}$ Racbel Howard, ${ }^{1}$ Michael G. Feblings, ${ }^{2}$ Fefferson R. Wilson. ${ }^{3}$ From the ${ }^{1}$ University College Cork, Cork, Ireland; ${ }^{2}$ University of Toronto, Toronto, Ont.; ${ }^{3}$ St. Michael's Hospital, Toronto, Ont.

Background: Despite objective clinical improvements following surgery for degenerative cervical myelopathy (DCM), experience dictates that a proportion of patients remain dissatisfied with their outcome. The learning objective is to investigate the presence of response shift in functional outcomes in DCM patients treated surgically. Methods: We enrolled 401 patients at 16 global sites. Functional impairment and self-reported rating of health status were recorded at baseline and 1-year after surgery. Concordance was defined as achieving a clinically meaningful improvement (minimal clinically important difference [MCID]) on the modified Japanese Orthopaedic Association (mJOA) scale and reporting general health as somewhat better or much better, whereas discordance was defined as achieving an MCID and reporting general health as the same, somewhat worse, or much worse. Logistic regression analysis was used to determine important differences between patients with discrepancies between their clinical measures and self-reported ratings and those without. Results: Based on patient self-reports, 55 patients were somewhat or much worse than 1 year prior, 82 patients were the same, and 264 patients were somewhat or much better. Thirtyone percent of patients who reported being somewhat or much worse achieved an MCID on the mJOA. In addition, 56\% of patients who indicated their health status was the same as 1 year prior exhibited clinically meaningful improvements in functional impairment. Univariate analyses indicated that a patient is more likely to be dissatisfied with their outcomes if he or she exhibited less improvement in mJOA upper extremity scores $(p=0.071)$, was older $(p=0.0073)$, was a smoker $(p=0.082)$, and achieved lower total mJOA scores at 1 year $(p=0.087)$. Following multivariate analysis, the most important factors were age and improvement in mJOA upper extremity scores. Conclusion: A response shift in functional outcomes was detected in surgical DCM patients. Older patients and those with smaller improvements in postoperative upper extremity scores tend to report worsened or unchanged general health status, despite experiencing improvements in overall function.

\section{2}

Degenerative cervical myelopathy in North America, 20022014: a silent epidemic. Fetan H. Badbiwala, ${ }^{1}$ Farshad Nassiri, ${ }^{1}$ Christopher D. Witiw, ${ }^{1}$ Mubammad Akbar, ${ }^{1}$ Alireza Mansouri, ${ }^{1}$ Saleb Almenawer, ${ }^{2}$ Michael G. Feblings, ${ }^{1}$ Fefferson R. Wilson. ${ }^{1}$ From the ${ }^{1}$ Division of Neurosurgery, 
Department of Surgery, University of Toronto, Toronto, Ont.; ${ }^{2}$ Division of Neurosurgery, Department of Surgery, McMaster University, Hamilton, Ont.

Background: Although degenerative cervical myelopathy (DCM) is purported to be the leading cause of spinal cord dysfunction globally, the population incidence of this clinicopathologic entity remains poorly defined. We sought to determine the incidence and health care resource use of surgical DCM at the population level, and compare these to other common neurosurgical pathologies using a large American national health care database. Methods: Patients who underwent surgery for DCM were identified from the Healthcare Cost and Utilization Project (HCUP) National Inpatient Sample (NIS) for years 2002 through 2014 using International Classification of Diseases, Ninth Revision, Clinical Modification (ICD-9-CM), codes. We calculated overall and age- and sex-stratified incidences per 100000 persons for each year using patient-specific discharge weights and population estimates from the United States Census Bureau. Trends for incidence rates were evaluated by joinpoint regression to determine the average annual percent change (AAPC). Incidences were also calculated for traumatic spinal cord injury (SCI) and 3 common neurosurgical procedures: evacuation of subdural hematoma (SDH) (including acute, subacute, and chronic), craniotomy for tumour (including primary and metastatic), and clipping or coiling of cerebral aneurysm. Results: The overall incidence of surgical DCM rose from 10.3 per 100000 in 2002 to 21.1 per 100000 in 2014, representing an AAPC of $5.9 \%$. The highest incidence $(61.1 / 100000)$ and growth rate (AAPC 7.4\%) were seen in the 65- to 84-year age group. Age-adjusted incidences and annual growth rates for DCM exceeded those of SCI $(5.0 / 100000 ;-0.5 \%)$, evacuation of SDH $(8.2 / 100000 ; 0.4 \%)$, craniotomy for brain tumour $(14.2 / 100000 ; 1.1 \%)$, and clipping or coiling of cerebral aneurysm $(7.2 / 100000 ; 2.4 \%)$. Cumulative hospital charges and costs related to surgery for DCM in 2014 were $\$ 1.14$ billion and \$291 million, respectively. Conclusion: Degenerative cervical myelopathy is a leading indication for surgery on the central nervous system. The incidence of, and health care dollars spent on, surgery for DCM has steadily risen over the past decade owing both to an aging population and increasing recognition of the role of surgical management.

\section{4}

Outcomes of surgical decompression in patients with very severe degenerative cervical myelopathy. Branko Kopjar, ${ }^{1}$ Parker Bobm, ${ }^{2}$ Goshua Arnold, ${ }^{3}$ Michael G. Feblings, ${ }^{4}$ Lindsay A. Tetreault, ${ }^{5}$ Paul Arnold, ${ }^{2}$ Fetan H. Badbiwala. ${ }^{4}$ From the ${ }^{1}$ University of Washington, Seattle, Wash.; ${ }^{2}$ Kansas University Medical Center, Kansas City, Kans.; ${ }^{3}$ Tufts University School of Medicine, Boston, Mass.; ${ }^{4}$ University of Toronto, Toronto, Ont.; ${ }^{5}$ University College Cork, Cork, Ireland.

Background: Although decompressive surgery has been determined to be a safe and effective approach for patients with myelopathic deficiencies, studies have suggested residual disability following treatment in patients with more severe disease presentation. The aim of this study was to evaluate outcomes of decompressive surgery in patients with very severe degenerative cervical myelopathy (DCM). Methods: We performed a prospective observational international study. Postoperative outcomes of 60 patients with very severe DCM (modified Japanese Orthopaedic Association $[\mathrm{mJOA}]$ score $=8$ ) were compared with outcomes of 188 patients with severe DCM $(\mathrm{mJOA}=9-11)$. Postimputation follow-up rate was $93.1 \%$. Unadjusted and adjusted analyses were performed using 2-way repeated-measures of covariance. Results: The 2 cohorts were similar in demographics, duration of myelopathy symptoms, source of stenosis, and surgical approaches used to decompress the spine. The very severe and severe cohorts differed in preoperative Nurick grades $(4.97 \mathrm{v}$. 3.91 , respectively, $p<0.0001$ ) and Neck Disability Index (NDI) scores (45.20 v. 56.21 respectively, $p=0.0006$ ). There were no differences in Short Form 36 (SF-36v2) physical (PCS) and mental (MCS) component summary scores. Both cohorts improved in mJOA, Nurick, NDI, and SF-36v2 PCS and MCS scores. Despite the substantial postoperative improvements, patients in both cohorts had considerable residual symptoms. Two-thirds of the patients in the very severe cohort had severe $(\mathrm{mJOA}=11)$ or moderate $(\mathrm{mJOA}=14)$ myelopathy symptoms at 24 months' follow-up. Longer duration of disease was associated with poorer treatment response. Conclusion: Decompressive surgery is effective in patients with very severe DCM; however, patients have significant residual symptoms and disability. The very severe subgroup $(\mathrm{mJOA}=8)$ of patients with DCM represents a distinct group of patients, and their different clinical trajectory is important for clinicians and patients to recognize. Duration of symptoms negatively affects chances for recovery. Whenever possible, patients with DCM should be treated before developing very severe symptomatology.

86

Radiographic outcome after cervical disc arthroplasty: development of heterotopic ossification. Michael M.H. Yang, ${ }^{1}$ Won Hyung A. Ryu, ${ }^{1}$ Stephan Du Plessis, ${ }^{1}$ Casha Steven, ${ }^{1}$ W. Bradley Facobs, ${ }^{1}$ R. Fobn Hurlbert. ${ }^{2}$ From the ${ }^{1}$ University of Calgary, Calgary, Alta.; ${ }^{2}$ University of Arizona, Tucson, Ariz.

Background: Cervical disc arthroplasty (CDA) has been widely used for select patients with degenerative disc disease. However, there are ongoing challenges to CDA including long-term durability to prevent adjacent segment disease (ASD), and development of heterotopic ossification (HO). The aim of this study was to examine radiographic characteristics of $\mathrm{CDA}$ in relation to the development of $\mathrm{HO}$ and the association between $\mathrm{HO}$ and ASD. Methods: A retrospective chart review was performed on patients who underwent 1- to 2-level CDA. For each patient, a minimum of 1 year of follow-up with serial cervical radiography was analyzed. Radiographs immediately after surgery and at follow-up were examined for HO (McAfee classification) and ASD (Hilibrand classification). Other measurements included global Cobb angle, global range-of-motion, adjacent level disc height, index level angular motion, and overhang of end plate in relation to artificial disc. Results: Thirty-five patients underwent CDA (mean age $47 \mathrm{yr} ; 20$ men and 15 women), with $74 \%$ receiving a 1-level procedure. Mean follow-up duration was 3.3 (range $1-8)$ years. Fifteen patients (43\%) had grade 3 or $4 \mathrm{HO}$, and 9 patients (26\%) had grade 2 or 3 ASD. Comparing the cohort of patients with high- and low-grade $\mathrm{HO}$, there was a significant difference in the length of end plate overhang $(1.31 \pm 1.76$ v. 2.59 
$\pm 1.44, t=3.25 ; p=002$ ). Both groups of patients maintained their global alignment, global range-of-motion, and angular motion at the index level for up to 8 years. There was no significant association between development of HO and ASD $\left(\chi^{2}=2.8 ; p=0.094\right)$. Comparing patients with and without ASD, patients with ASD had significant kyphotic changes in their alignment $\left(5^{\circ}\right)$ unlike those without ASD $\left(-2^{\circ} ; t=-2.33 ; p=0.03\right)$. Conclusion: Patients with greater than $2 \mathrm{~mm}$ of overhang of end plate in relation to the artificial disc may be at higher risk of developing HO. The relationship between development of $\mathrm{HO}$ and ASD remains unclear. However, change in sagittal alignment may predict radiographic ASD.

\section{7}

Prediction of anterior versus posterior surgical approach for degenerative cervical myelopathy based on magnetic resonance imaging pathology: analysis of a global cohort. Aria Nouri, ${ }^{1}$ Allan R. Martin, ${ }^{2}$ So Kato, ${ }^{2}$ Christopher D. Witiw, ${ }^{2}$ Anick Nater, ${ }^{2}$ Lindsay A. Tetreault, ${ }^{2}$ Carlo Santaguida, ${ }^{2}$ David Gimbel, ${ }^{1}$ Rani Nasser, ${ }^{1}$ Joseph Cheng, ${ }^{1}$ Hamed Reibani-Kermani, ${ }^{2}$ Michael G. Feblings. ${ }^{2}$ From the ${ }^{1}$ University of Cincinnati, Cincinnati, Ohio; ${ }^{2}$ University of Toronto, Toronto, Ont.

Background: Degenerative cervical myelopathy (DCM) is the most common cause of spinal cord compression (SCC) and impairment. Surgical treatment is usually undertaken, but the decision between an anterior or posterior approach as the optimal choice is often controversial. To better understand this surgical decision-making process, we analyzed imaging data from 2 multicentre prospective studies. Methods: We analyzed magnetic resonance images (MRIs) of 458 patients for various pathologic features, characteristics of SCC, sagittal alignment using the modified K-line, and signal changes on T1-weighted (T1WI) and T2-weighted (T2WI) images. Clinical severity was measured with modified Japanese Orthopaedic Association (mJOA) score. Fisher exact tests and multivariate logistic regression were used to assess relationships between clinical/MRI features and anterior, posterior, or combined surgical approach. Results: Operative approach was 265 anterior, 184 posterior, and 9 combined. Anterior surgery was favoured with lower age, $\mathrm{mJOA} \geq 15$, single-level disc pathology, anterior-only SCC, and kyphosis, whereas a posterior approach was favoured in South America or with multilevel spondylosis, ligamentum flavum enlargement, spondylolisthesis, SCC at or above C4-C5, SCC at or below C6-C7, more levels with SCC, T2WI hyperintensity, and more levels with T2WI hyperintensity (all $p<0.05)$. In multivariate analysis, levels with SCC $(p<$ $0.001)$, age $(p<0.001)$, South American region $(p<0.001)$, mJOA $\geq 15(p=0.008)$, kyphosis $(p=0.02)$, and maximal SCC at or above C4-C5 ( $p=0.05)$ were significant independent predictors. Models based on clinical and MRI factors predicted anterior or posterior approach with $78 \%$ and $80 \%$ accuracy, respectively, whereas a combined model achieved $87 \%$ accuracy. Conclusion: Clinical and MRI factors are independently able to predict surgical approach in DCM, and a combined model has excellent predictive capacity. The predictive utility of specific MRI factors on surgical approach corroborated expert opinion, and this study has provided information regarding the magnitude of their influence. These findings may be used in conjunction with machine learning to develop surgical treatment algorithms for DCM.
90

Using a machine learning approach to predict outcome after surgery for degenerative cervical myelopathy. Zamir Merali, ${ }^{1}$ Jetan H. Badbiwala, ${ }^{1}$ Christopber D. Witiw, ${ }^{1}$ Michael G. Feblings. ${ }^{1,2}$ From the ${ }^{1}$ University of Toronto, Division of Neurosurgery, Toronto, Ont.; ${ }^{2}$ Toronto Western Hospital, Division of Neurosurgery, Toronto, Ont.

Background: Decision-making within spine surgery is challenging owing to heterogeneous patient populations and variability in disease presentation. Spine surgeons must synthesize a large quantity of complex information to make an accurate prediction of a patient's response to a given intervention. Recent increases in computing power have made it possible to extract patterns from large clinical data sets and develop predictive models to assist medical decision-making. We used a machine learning approach to predict outcomes after surgery for degenerative cervical myelopathy (DCM). Methods: Patients undergoing surgery for DCM as part of the AOSpine Cervical Spondylotic Myelopathy North American (CSM-NA) or International (CSM-I) trials were included in the study. Predictor variables reflected information about preoperative disease severity, disease presentation, radiographic features, patient demographics, and comorbidities. The outcome was an improvement in the modified Japanese Orthopaedic Association (mJOA) score by at least the minimum clinically important difference (MCID). A machine-learning approach of feature engineering, data preprocessing, and model optimization was used to create the most accurate predictive model of outcome after surgery for DCM. Models were evaluated against a separate testing patient cohort that was not used for model development. Results: Out of 757 patients, 562 had complete information at follow-up and were included in the analysis. Following data preprocessing, 58 predictors were chosen for model training. The best predictive model used a random forest structure and had a mean area under the curve (AUC) of 0.70 , sensitivity of $83 \%$, and specificity of $51 \%$. This model was secondarily validated on an independent testing cohort and achieved an AUC of 0.70 , sensitivity of $78.1 \%$, and specificity of $55 \%$. A logistic regression model had an AUC of 0.56 , sensitivity of $51 \%$ and specificity of $63 \%$. Conclusion: A machine-learning approach was able to provide accurate prediction of improvement in the mJOA score after surgery for DCM. This study demonstrates that a machine-learning approach can produce a predictive model that may be used to assist clinical decision-making.

\section{9}

Error propagation in spinal intraoperative navigation from nonsegmental registration: a prospective cadaveric and clinical study. Daipayan Guba, ${ }^{1}$ Raphael Fakubovic, ${ }^{2}$ Albert Yee, ${ }^{3}$ Victor Yang. ${ }^{1}$ From the ${ }^{1}$ Division of Neurosurgery, University of Toronto, Toronto, Ont.; ${ }^{2}$ Department of Biomedical Physics, Ryerson University, Toronto, Ont.; ${ }^{3}$ Division of Orthopedic Surgery, University of Toronto, Toronto, Ont.

Background: Computer-assisted navigation may guide spinal instrumentation. Current systems rely on a dynamic reference frame (DRF) for image-to-patient registration. Here, we quantify navigation inaccuracy due to distance from the registered level, surgical manipulation, and intraoperative patient respiration- 
induced vertebral motion. Methods: Navigation error due to distance from the DRF and vertebral motion during screw tract formation were quantified in 4 human cadavers. An optical navigation system was registered through a posterior midline exposure. Bone screws were implanted into the laminae bilaterally from $\mathrm{C} 2$ to $\mathrm{S} 1$. The tip of a tracked awl was placed into the screw head at each level, at 0-5 levels distant from the registered level, and the tool tip position compared with that of the screw head on postprocedure computed tomography. Respirationinduced vertebral motion was quantified from 13 in vivo clinical cases of open posterior instrumented fusion. The 3-dimensional (3D) position of a spinous-process clamp was tracked over 12 respiratory cycles. Results: Significant increases in 3D navigation error were seen with increasing distance from the registered level, greater than $2 \mathrm{~mm}$ at 3 or more levels from the DRF. The increase in $3 \mathrm{D}$ error was predominantly in the mediolateral axis $(1.78 \pm 0.86 \mathrm{~mm}, 2.78 \pm 0.86 \mathrm{~mm}, 2.19 \pm 0.92 \mathrm{~mm}$, and $3.08 \pm$ $0.89 \mathrm{~mm}$, at 2, 3, 4 and 5 levels from the DRF, respectively) and anteroposterior axis $(1.40 \pm 0.81 \mathrm{~mm}, 1.79 \pm 0.81 \mathrm{~mm}, 2.18 \pm$ $0.87 \mathrm{~mm}$, and $4.30 \pm 0.84 \mathrm{~mm}$, at $2,3,4$ and 5 levels from the DRF). Manipulation during screw tract formation caused displacement predominantly in the mediolateral $(0.71 \pm 0.84 \mathrm{~mm})$ and craniocaudal planes $(1.02 \pm 0.92 \mathrm{~mm})$. Respiration-induced absolute vertebral motion was maximal in the anteroposterior $(2.42 \pm 1.77 \mathrm{~mm})$ and craniocaudal axes $(0.92 \pm 0.69 \mathrm{~mm})$. In multivariate regression, both tidal volume and end-expiratory pressure were positively correlated with anteroposterior and 3D displacement. Conclusion: Although respiration- and manipulation-induced vertebral motion are small, they can be greater than $2 \mathrm{~mm}$ in up to $37 \%$ of cases. Surgeons may mitigate these errors intraoperatively by placing the DRF adjacent to the registered level; temporary apnea may be warranted at critical stages.

\section{9}

Role of the sodium-glutamate blocker riluzole in enhancing functional outcomes in patient undergoing surgery for degenerative cervical myelopathy: results of the prospective, multicentre double blind controlled cervical spondylotic myelopathy-protect randomized controlled trial. Michael G. Feblings, ${ }^{1}$ Branko Kopjar, ${ }^{2}$ Henry Abn, ${ }^{3}$ Francis Farbadi, ${ }^{4}$ Christopher Shaffrey, ${ }^{5}$ Abmad Nassr, ${ }^{6}$ Praveen Mummaneni, ${ }^{7}$ Paul Arnold, ${ }^{8}$ Bradley Facobs, ${ }^{9}$ K. Daniel Riew, ${ }^{10}$ Darrel Brodke, ${ }^{11}$ Alexander Vaccaro, ${ }^{12}$ Alan Hilibrand, ${ }^{12}$ Jason Wilson, ${ }^{13}$ James Harrop, ${ }^{12}$ S. Tim Yoon, ${ }^{14}$ Kee Kim, ${ }^{15}$ Daryl Fourney, ${ }^{16}$ Carlo Santaguida, ${ }^{17}$ Jetan Badbiwala. ${ }^{1}$ From the ${ }^{1}$ University of Toronto, Toronto, Ont.; ${ }^{2}$ University of Washington, Seattle, Wash.; ${ }^{3}$ St. Michael's Hospital, Toronto, Ont.; ${ }^{4}$ Ohio State University, Columbus, Ohio; ${ }^{5}$ University of Virginia, Charlottesville, Va.; ${ }^{6}$ Mayo Clinic, Rochester, NY; ${ }^{7}$ University of California at San Francisco, San Francisco, Calif.; ${ }^{8}$ Kansas University Medical Center, Kansas City, Kans.; ${ }^{9}$ University of Calgary Spine Program, Calgary, Alta.; ${ }^{10}$ Columbia University, New York City, NY; ${ }^{11}$ University of Utah, Salt Lake City, Utah; ${ }^{12}$ Thomas Jefferson University and Rothman Institute Orthopaedics, Philadelphia, Penn.; ${ }^{13}$ Louisiana State University, New Orleans, La.; ${ }^{14}$ Emory University, Atlanta, Ga.; ${ }^{15}$ University of California Davis Spine Center, Sacramento, Calif.; ${ }^{16}$ University of Saskatchewan Royal Univer- sity Hospital, Saskatoon, Sask.; ${ }^{17}$ McGill University Health Centre, Montreal, Que.

Background: Degenerative cervical myelopathy (DCM), which encompasses cervical spondylotic myelopathy and ossification of the posterior longitudinal ligament, is the most common cause of spinal cord impairment. Decompressive surgery is the most effective treatment; however, most patients are left with residual neurologic impairment, and some patients experience neurologic decline. Based on strong preclinical basic science evidence and collateral evidence from trials in human spinal cord injury and amyotrophic lateral sclerosis, we sought to explore if the sodiumglutamate antagonist riluzole would enhance neurologic recovery and reduce perioperative neurologic decline. Methods: This is a phase III multicentre, double-blind, placebo-controlled, randomized controlled trial. Between March 2012 and June 2017, 300 surgically naive patients with moderate to severe DCM were enrolled at 16 sites. Subjects were randomized 1:1 to either the $50 \mathrm{mg}$ riluzole bid or placebo-controlled group, beginning the medications 14 days presurgery and ending 28 days postsurgery. Follow-up was at 6- and 12 months to determine the primary end point (change in modified Japanese Orthopaedic Association [mJOA] scores) and the secondary end points (change in shortform 36 [SF-36v2], Neck Disability Index [NDI], Nurick grade, EuroQol 5-dimension [EQ-5D], American Spinal Injury Association [ASIA] motor and sensory scores, Bazaz scale, visual analogue scale [VAS] for pain, grip strength and neurologic compilations. Results: The mean age was 57.9 years (standard deviation [SD] 10.2$) ; 55.7 \%$ of patients were male; $80.1 \%$ of patients were white; $9.7 \%$ of patients were black. Baseline mJOA score was 11.84 (SD 1.5, range 8-14); Nurick grade was 3.3 (SD 0.8); NDI was 42.9 (SD 13.5); VAS arm/shoulder was 4.7 (SD 2.9); VAS neck was 4.9 (SD 3.0). The study will be completed in December 2017 , with a projected follow-up rate exceeding $90 \%$. Efficacy results will be available and presented at the conference. Conclusion: This study contributes level I evidence concerning efficacy and safety of riluzole as an adjunct therapy to decompressive surgery for patients with DCM.

8

Quantification of computational geometric congruence in surface-based registration for spinal intra-operative threedimensional navigation. Daipayan Guba, Raphael Fakubovic, Victor Yang. From the Sunnybrook Research Centre, Toronto, Ont.

Background: Computer-assisted navigation (CAN) may guide spinal instrumentation and requires alignment of patient anatomy to imaging. Iterative-closest-point algorithms register anatomic and imaging data sets, which may fail in the presence of significant geometric symmetry (congruence), leading to failed registration or inaccurate navigation. We computationally quantify geometric congruence in posterior spinal exposures, and identify predictors of potential navigation inaccuracy. Methods: Midline posterior exposures were performed from $\mathrm{C} 1-\mathrm{S} 1$ in 4 human cadavers. An optically based CAN generated surface maps of the posterior elements at each level. Maps were reconstructed to include bilateral hemilamina or unilateral hemilamina with and without the base of the spinous process. Maps were fitted to symmetric geometries (cylindrical, spherical, planar) using 
computational modelling, and the degree of model fit was quantified based on the ratio of model inliers to total points. Geometric congruence in a clinical setting was assessed similarly in 11 patients undergoing midline exposures in the cervical/thoracic/ lumbar spine for posterior instrumented fusion. Results: In cadaveric testing, increased cylindrical/spherical/planar symmetry was seen in the subaxial cervical spine relative to the high-cervical and thoracolumbar spine $(p<0.001)$. Inclusion of the base of the spinous process decreased symmetry independent of spinal level $(p<0.001)$. Registration with bilateral compared with unilateral hemilamina did not significantly reduce geometric symmetry. In clinical testing, increased cylindrical/spherical/planar symmetry was again seen in the subaxial cervical spine relative to the thoracolumbar spine $(p<0.001)$, and in the thoracic spine relative to the lumbar spine $(p<0.001)$. Symmetry in all geometries was decreased by $20 \%$ with inclusion of the base of the spinous process as opposed to without. Conclusion: Geometric congruence is most evident at $\mathrm{C} 1$ and the subaxial cervical spine, warranting greater vigilance in navigation accuracy verification. At all levels, inclusion of the base of the spinous process in unilateral registration decreases the likelihood of geometric symmetry and navigation error, important for minimally invasive unilateral approaches.

71

Upright open magnetic resonance imaging for lumbar spinal musculature in varying postures: a pilot study. Noor Shaikh, John Street, Hanbing Zhang, David Wilson, Thomas Oxland. From the Division of Spine, Department of Orthopaedics, University of British Columbia, Vancouver, BC.

Background: Lumbar spinal musculature is critical for equilibrium, stability, and control of the spine. Typically, model validation uses supine magnetic resonance imaging (MRI), which may not be representative of upright postures. This study's objective was to characterize lumbar spinal musculature in varying postures using upright open MRI. Methods: Six healthy volunteers were imaged in a $0.5 \mathrm{~T}$ vertical open MRI scanner using a T1-weighted Spin Echo sequence. Each volunteer was scanned in 7 postures: standing, standing holding $8 \mathrm{~kg}$, standing bent $45^{\circ}$ forward, seated bent $45^{\circ}$ forward, seated at $90^{\circ}$, seated bent $45^{\circ}$ back, and supine. For each posture, 3 slices were taken parallel to the disc at each intervertebral level: L3/L4, L4/L5, and L5/S1. We took measurements of muscle cross-sectional area (CSA), centroid, radius, and angle for the multifidus and erector spinae combined, and for the psoas major. Muscle CSA $\left(\mathrm{mm}^{2}\right)$ was determined. Angle $\left(^{\circ}\right)$ was relative to the spinous process, and radius $(\mathrm{mm})$ was the distance between the muscle and vertebral body centroids. The effect of posture was evaluated with a repeated-measures analysis of variance $(p<0.05)$. Posture repeatability was evaluated by comparing muscle CSA in 4 rescanned volunteers. Repeatability measures were assessed on muscle CSA by intraclass correlation coefficient (ICC). Results: Posture affected the right and left psoas major CSA at L3/L4. The largest differences in CSA were seen when seated bent $45^{\circ}$ forward, which was $51.7 \%$ greater than supine (right), and seated at $90^{\circ}$, which was $60.5 \%$ greater than supine (left). Posture also affected the multifidus and erector spinae angle at L3/L4. The muscle CSA, radius, and angle of all other levels and muscles were different but not statistically significant. Posture repeatability $\operatorname{ICC}(3,1)$ was $0.59-0.92$ for the multifidus and erec- tor spinae, and $0.77-0.93$ for the psoas major. Intrarater reliability was $0.96-0.98$ for the multifidus and erector spinae, and 0.89-0.93 for the psoas major. Conclusion: We observed a number of differences in spinal muscle morphology and anatomy in different positions. Intrarater reliability was excellent despite the lower resolution compared with closed-bore MRI scanners (1.5-3 T). Studies with more volunteers and posture repeatability should be considered before incorporating such results to inform more accurate lumbar spine models.

56

Relationship between cervical muscle morphology evaluated by magnetic resonance imaging, cervical muscle strength, and functional outcomes in patients with degenerative cervical myelopathy. Maryse Fortin, ${ }^{1}$ Nikola Wilk, ${ }^{2}$ Octavian Dobrescu, ${ }^{2}$ Philippe Martel, ${ }^{2}$ Carlo Santaguida, ${ }^{2}$ Michael Weber, ${ }^{2}$ Elie Akoury. ${ }^{3}$ From the ${ }^{1}$ PERFORM Centre, Concordia University, Montreal, Que.; ${ }^{2}$ McGill University Health Centre, Montreal, Que.; ${ }^{3}$ Montreal General Hospital, Department of Experimental Surgery, Division of Orthopaedic Surgery, Montreal, Que.

Background: Cervical muscle alterations have been reported in patients with chronic neck pain, but the assessment of muscle morphology and strength has been overlooked in patients with degenerative cervical myelopathy (DCM). This study aimed to investigate the relationship between cervical muscle degenerative changes observed on magnetic resonance imaging (MRI), muscle strength, symptom severity, and functional status in patients with DCM. Methods: A total of 20 patients diagnosed with DCM were included in this study. Quantitative cervical muscle measurements of the multifidus, semispinalis cervicis, semispinalis capitis, and splenius capitis, including total cross-sectional area (CSA), functional CSA (FCSA, fat-free area) and ratio of FCSA to CSA (e.g., indicator of muscle fatty infiltration) were obtained from T2-weigthed axial MRI from C2-C3 to C6-C7. Muscle strength was assessed using a microFET 2 hand-held dynamometer in flexion, extension, right-side bending, and left-side bending. The association between cervical muscle morphology parameters measured on MRI, muscle strength, symptom severity, and functional status was investigated using multivariate linear regression models. Results: Greater mean CSA and FCSA were associated with greater overall muscle strength. The mean FCSA explained $33 \%, 74 \%, 35 \%, 15 \%$, and $61 \%$ of the total variance in flexion, extension, right-side bending, left-side bending, and overall muscle strength, respectively. The mean ratio of FCSA to CSA was not significantly associated with cervical muscle strength in any position. None of the MRI muscle morphology parameters were significantly associated with symptom severity or duration. However, greater mean FCSA:CSA (less fatty infiltration) was associated with higher modified Japanese Orthopaedic Association score (e.g., lower disability) $\left(p=0.02, R^{2}=0.15\right)$. Conclusion: Cervical lean muscle mass (FCSA) was positively associated with cervical muscle strength in patients with DCM. Moreover, greater fatty infiltration in the cervical extensor muscles was associated with lower functional score. Such findings suggest that clinicians should pay greater attention to cervical muscle morphology and function in patients with DCM. Future studies should further evaluate the effects of specific rehabilitative cervical muscle strengthening exercises on CSA and FCSA in patients with DCM. 
10

Optical topographic imaging for spinal intraoperative 3-dimensional navigation in minimally invasive approaches: initial preclinical and clinical feasibility. Daipayan Guba, Raphael Fakubovic, Albert Yee, Michael G. Feblings, Victor Yang. From the University of Toronto, Toronto, Ont.

Background: Computer-assisted 3-dimensional navigation may guide spinal instrumentation. A novel optical topographic imaging (OTI) system, developed in our laboratory, offers comparable accuracy and significantly faster registration relative to current navigation systems, in open posterior thoracolumbar exposures. Here, we explore the utility of OTI in minimally invasive (MI) spinal approaches. Methods: Mini-open midline posterior exposures were performed in 4 human cadavers. Square exposures of $25,30,35$, and $40 \mathrm{~mm}$ were registered to a preoperative computed tomography (CT) scan. Screw tracts were fashioned using a tracked awl and probe, and instrumentation placed. Navigation data were compared with screw positions on postoperative CT imaging, and absolute translational and angular deviations computed. In vivo validation was performed in 8 patients, with mini-open thoracolumbar exposures and percutaneous placement of navigated instrumentation. Results: For 37 cadaveric screws, absolute translational errors were $1.79 \pm 1.43 \mathrm{~mm}$ and $1.81 \pm 1.51 \mathrm{~mm}$ in the axial and sagittal planes, respectively; absolute angular deviations were $3.81^{\circ} \pm$ $2.91^{\circ}$ and $3.45^{\circ} \pm 2.82^{\circ}$, respectively. Errors were similar across levels and screw types. The number of surface points registered by the navigation system, but not exposure size, correlated positively with the likelihood of successful registration (odds ratio [OR] 1.02, 95\% confidence interval [CI] 1.009-1.024, $p<$ 0.0001 ). We analyzed 55 in vivo thoracolumbar pedicle screws. Overall axial and sagittal translational errors were $1.79 \pm$ $1.41 \mathrm{~mm}$ and $2.68 \pm 2.26 \mathrm{~mm}$, respectively, whereas axial and sagittal angular errors were $3.63^{\circ} \pm 2.92^{\circ}$ and $4.65^{\circ} \pm 3.36^{\circ}$, respectively. There were no critical radiographic breaches, nor were there any neurovascular complications from any placed screws. Conclusion: Optical topographic imaging is a novel navigation technique previously validated for open posterior exposures, which has comparable accuracy for mini-open MI exposures. The likelihood of successful registration is affected more by the geometry of the exposure than by its size.

\section{4}

Early decompression strategy is effective in thoracolumbar traumatic spinal cord injury with lower-grade residual spinal canal compression. Fulien Goulet, ${ }^{1}$ Fean-Marc Mac-Thiong. ${ }^{1,2}$ From the ${ }^{1}$ Université de Montréal, Montréal, Que.; ${ }^{2}$ Hôpital du Sacré-Coeur de Montréal, Montréal, Que.

Background: After traumatic spinal cord injury (tSCI) at the thoracolumbar level, the potential for neurologic recovery and related prognostic factors are subject to many studies and controversies. Early spinal canal decompression tends to favour neurologic outcome, but no study has evaluated the role of residual spinal canal compression in early surgery strategies. The objective of this study was to determine the influence of surgical timing on neurologic recovery in relationship with the residual spinal canal compression documented after tSCI.
Methods: A prospective cohort of 42 patients that had sustained a tSCI from T10 to L2, and consecutively admitted to a single level 1 SCI-specialized trauma centre between 2010 and 2016, was studied. Patients were stratified according to surgical timing ( $<24$ or $>24 \mathrm{~h}$ posttrauma). Maximum canal compromise (MCC) and maximum spinal cord compression (MSCC) were calculated for all patients. Neurologic recovery was dichotomized and considered present if improvement was documented on the American Spinal Injury Association (ASIA) scale at follow-up of a minimum of 6 months. Nonparametric tests were applied to assess difference in prevalence of neurologic recovery in different subgroups. Results: There was no difference in neurologic recovery between patients who underwent surgery within 24 hours and patients who underwent surgery more than 24 hours from trauma. Maximum canal compromise and MSCC alone did not influence neurologic outcome. When operated on within 24 hours, patients who had a lower level of MCC $(<50 \%)$ showed comparable neurologic recuperation with patients with higher level of compression (> 50\%). Similar results were obtained when repeating the analyses with threshold of $66 \%$ or more of residual compression, and when using MCC as a continuous variable. Neurologic recovery in patients who underwent surgery within 24 hours did not differ according to different levels of MSCC. Conclusion: With demonstration of similar neurologic outcomes at 6 months, patients with low-grade residual spinal canal compression benefit from early decompression strategy similar to patients with higher-grade residual compression. Prevalence of neurologic recovery was not different in groups who underwent surgery within 24 hours, regardless of stratification for level of MCC or MSCC.

\section{1}

$\alpha$-Amino-3-hydroxy-5-methyl-4-isoxazolepropionic acid receptor modulation as a therapeutic strategy to enhance survival of spinal cord neural stem cells. Laureen Hachem, ${ }^{1,2}$ Andrea Mothe, ${ }^{2}$ Charles Tator. ${ }^{1,2,3}$ From the ${ }^{1}$ Division of Neurosurgery, University of Toronto, Toronto, Ont.; ${ }^{2}$ Krembil Research Institute, Toronto Western Hospital, Toronto, Ont.; ${ }^{3}$ Division of Neurosurgery, Toronto Western Hospital, Toronto, Ont.

Background: Transplantation of neural stem and progenitor cells (NSPCs) following spinal cord injury (SCI) is a promising strategy to enhance regeneration, but it is limited by poor survival of grafted cells. Recently, we demonstrated for the first time that the excitatory neurotransmitter glutamate, which is released after SCI, promotes survival and proliferation of adult spinal cordderived NSPCs via the $\alpha$-amino-3-hydroxy-5-methyl-4isoxazolepropionic acid (AMPA) subtype of glutamate receptors. In the present study, we examined the therapeutic potential of selective AMPA-receptor modulation on NSPC survival using a class of allosteric AMPA-receptor modulators known as ampakines. Methods: Neural stem and progenitor cells isolated from the periventricular region of the adult rat spinal cord were exposed to ampakines CX546 or CX614 for 72 hours, either alone or in the presence of low-dose $(50 \mu \mathrm{mol} / \mathrm{L})$ glutamate (to mimic glutamate concentrations in the injured spinal cord during the subacute period). To examine the effects of ampakineglutamate treatment in the setting of oxidative stress, NSPCs were concurrently exposed to a high dose of hydrogen peroxide 
(500 $\mu \mathrm{mol} / \mathrm{L}$ for $6 \mathrm{~h})$. Results: Treatment with CX546 or CX614 in the presence of glutamate led to a significant increase in live cell numbers compared with controls. This effect was due to both a reduction in cell death and increase in cellular proliferation. Moreover, ampakine-glutamate treatment led to a significant increase in cell survival compared with controls in the setting of oxidative stress. Treatment with ampakines did not significantly alter cell phenotype. Conclusions: We present the first examination of the effect of allosteric AMPA receptor modulators on adult spinal cord-derived NSPCs. Positive modulation of AMPA receptors may be a promising therapeutic strategy in the subacute or chronic phases after SCI to increase survival of endogenous or transplanted NSPCs.

\section{6}

Safety and efficacy of silver alloy-coated urinary catheters in patients with acute traumatic cervical spinal cord injury. Hanbing Zhou, Leanna Ritchie, Lise Belanger, Brian Kwon, Tamir Ailon, Nicolas Dea, Marcel Dvorak, Charles Fisher, Scott Paquette, Fobn Street. From the Division of Spine, Department of Orthopaedics, University of British Columbia, Vancouver, BC.

Background: Patients with acute traumatic cervical spinal cord injury (ATCSCI) have an increased risk of catheter-associated urinary tract infection (CAUTI). The safety and efficacy of antiseptic silver alloy-coated silicone urinary catheters (SACC) in ATCSCI is unknown. This study examined the safety and efficacy of SACC for preventing CAUTI in patients with ATCSCI. Methods: We performed a longitudinal observational study examining all CAUTI incidents in all patients undergoing spine surgery at a single quaternary centre from January 2014 to December 2016. Before July 2015, all patients received a latex indwelling catheter (LIC). After July 2015, SACC was used in all patients with ATCSCI only. Duration of catheterization, microbiology, duration of infection, antibiotic susceptibility, and catheter-associated adverse events were recorded prospectively, as was the occurrence of other noncatheter adverse events and length of stay. The safety and efficacy of SACC compared with LIC is reported. Results: We included 3081 patients, of whom $302(9.8 \%)$ had ATCSCI; $63 \%$ of ATCSCI patients were ASIA Impairment Scale (AIS) A or B. The overall rate of CAUTI was $19 \%(585 / 3081)$, with $38 \%(116 / 302)$ in patients with ATCSCI. Of 178 ATCSCI patients with LIC, 100 (56\%) developed a CAUTI compared with of 28 of 124 (23\%) patients with SACC $(p<0.05)$. The median time of urethral catheterization was 27 days in the SACC group and 28 days in the LIC group $(p=$ $0.602)$. Polymicrobial and gram-positive infection was more common in LIC than in SACC groups $(p<0.05)$. The median duration of infection was 9 days in the SACC group and 12 days in the LIC group $(p=0.08)$. Resistance to trimethoprim $(p<0.001)$ and ciprofloxacin $(p<0.05)$ were more common in LIC group. There was no difference in catheter-associated adverse events or length of stay between the groups. Conclusion: This study illustrates the safety and efficacy of antiseptic silver alloy-coated silicone urinary catheters in patients with ATCSCI. The use of SACC reduces the incidence and duration of CAUTI and the incidence of antibiotic resistance. This study provides the preliminary data for a prospective randomized controlled trial in patients with neurogenic bladder.
80

Acute complications after spinal cord injury: characteristics and the relation to long-term neurologic recovery and functional outcome in the North American Clinical Trials Network for Spinal Cord Injury longitudinal study cohort Blessing N.R. Faja, ${ }^{1}$ Fan Fiang, ${ }^{1}$ Michael G. Feblings, ${ }^{1}$ Jefferson R. Wilson. ${ }^{2}$ From the ${ }^{1}$ Divisions of Neurosurgery, Orthopedic Surgery and Spinal Program, University of Toronto and Toronto Western Hospital, Toronto, Ont.; ${ }^{2}$ St. Michael's Hospital, Division of Neurosurgery, University of Toronto, Toronto, Ont.

Background: There is a paucity of prospective multicentre series on the spectrum of acute complications and the impact on long-term outcomes in patients with traumatic spinal cord injury (SCI). This study investigated the North American Clinical Trials Network (NACTN) cohort to describe the spectrum of acute complications and the impact on long-term neurologic recovery and functional outcomes. Methods: Descriptive statistics were applied to examine the incidence, type, and distribution of acute complications in 801 patients from the NACTN database. Penalized maximum logistic and negative binomial regression models were fitted to estimate the factors associated with higher incidence of complications and to estimate the likelihood of neurologic recovery (American Spinal Injury Association (ASIA) Impairment Scale (AIS) improvement $\geq 1$ point) and functional outcome according to the Spinal Cord Independence Measure (SCIM) subscores for respiration, bladder, and bowel sphincter management; as well as that for indoor mobility 6 months after the injury. Results: A total of 1334 complications were recorded in 502 patients who experienced at least 1 complication during hospital admission (63\% of the study population). The most frequent complications were pneumonia (218 occurrences), respiratory failure (207), anemia (197), urinary tract infection (139), pleural effusion (92), bradycardia (93), depression (89), and sacral ulcers (72). Patients with acute complications were significantly more likely to have a complete injury at baseline assessment $(85.3 \%$ v. $44.5 \%$; $p<0.001)$. The occurrence of a complication doubled the average length of hospital stay $(18 \mathrm{v} .9 \mathrm{~d}$, $p<0.001)$. At 6 months' follow-up, patients who experienced at least 1 complication were more likely to require assistance with breathing (odds ratio [OR] 6.29, 95\% confidence interval [CI] 1.08-36.47), require support with bladder (OR 4.71, 95\% CI 2.32-9.58) or bowel (OR 3.35, 95\% CI 1.72-6.53) sphincter management, and require support with ambulation (OR 3.43, 95\% CI 1.90-6.19). The group with acute complications were less likely to experience AIS grade improvement of 1 or more points at 6 months (OR 0.55 , 95\% CI 0.32-0.96). Conclusion: Secondary medical complications are very common after SCI. These results are of importance, given the increasing evidence that acute complications can impact longterm functional outcomes. The results of this study may enable targeted efforts to prevent and attenuate the impact of acute complications following SCI.

\section{3}

Community-acquired pressure injuries in work-related spinal cord injury population. Barry White, ${ }^{1}$ fo-Anne Chisholm, ${ }^{2}$ Carly Rivers. ${ }^{1}$ From the ${ }^{1}$ Rick Hansen Institute, 
Vancouver, BC; ${ }^{2}$ Access Community Therapists Ltd., Vancouver, BC.

Background: The analysis is intended to enhance understanding of the problem of community-acquired pressure injuries (CAPIs) in the spinal cord injury (SCI) population and establish evidence of return on investment in a proactive solution offered to people with work-related SCI. Further evidence in SCI populations in Canada will support analyses of demand for solutions, as well as economic analyses of solutions, with an eye toward achievement of efficiencies in the supply of health care. Methods: We used retrospective data from a case series of 244 people with workrelated SCI who received preventative and early intervention, featuring comprehensive CAPI assessment by experienced health professionals between July 15, 2011, and Jan. 1, 2015, funded by WorkSafeBC, to describe the problem of CAPIs. Employing an activity-based costing methodology, detailed WorkSafeBC claims data were linked to assessment data and used to estimate the cost of CAPIs from the perspective of WorkSafeBC. Data from follow-up assessment of a subgroup of participants were used to examine economic benefits of intervention. Results: About 34\% of participants had 1 or more CAPIs at initial assessment. The mean cost of CAPIs (stages I-IV and unstageable) was estimated to be $\$ 56092$ in $2015 \mathrm{CAD}$. The mean cost ranged from $\$ 9580$ for stage I to \$238 736 for stage IV. Assuming that injury progression is likely in the absence of informed intervention, timely detection of stage I or II CAPIs provided an opportunity to avoid excess cost of more than $\$ 2.5$ million. Experiences at follow-up suggest that intervention was effective in preventing progression and occurrence or reoccurrence of pressure injuries, translating into cost avoidance and a positive net benefit. Reductions in the prevalence, mean number, and risk of pressure injuries were also observed. Conclusion: This analysis provides evidence of the problem of pressure injuries in an SCI population and evidence to support the benefits of a proactive solution to a problem in an area of care commonly described as reactive. Evidence is relevant to decisions (regional, provincial) regarding the provision of health care.

72

Relationships between specific functional abilities and health-related quality of life in chronic spinal cord injury. Fulien Goulet, ${ }^{1}$ Andréane Richard-Denis, ${ }^{2}$ Cynthia Thompson, ${ }^{2}$ Fean-Marc Mac-Thiong. ${ }^{1,2}$ From the ${ }^{1}$ Université de Montréal, Montréal, Que.; ${ }^{2}$ Hôpital du Sacré-Coeur de Montréal, Montréal, Que.

Background: Traumatic spinal cord injury (tSCI) is a debilitating condition that leads to important challenges for patients and that affects health-related quality of life (HRQoL). Qualitative research has shown that motor function and sphincter management are significant factors influencing HRQoL following tSCI. No study has objectively assessed which specific functional abilities are most important in the HRQoL of patients in the chronic phase of tSCI. The objective of this study was to explore the relationships between specific functional abilities assessed from the 3 rd version of the Spinal Cord Injury Measure (SCIM), and HRQoL assessed by the short-form 36 (SF-36v2) following tSCI. Methods: A prospective cohort of 195 patients that had sustained a tSCI from $\mathrm{C} 1$ to $\mathrm{L} 1$ consecutively admitted to a single level 1
SCI-specialized trauma centre between April 2010 and September 2016 was studied. The SCIM and SF-36v2 questionnaires were administered concurrently during routine follow-up visits between 6 and 12 months after the trauma. Correlation coefficients were calculated between SCIM scores (total, subgroups, and individual items scores), and SF-36v2 summary scores (physical component score, PCS; mental component score, MCS). All analyses were repeated separately for subjects with tetraplegia and paraplegia. Results: The total SCIM score correlated moderately with the PCS in the entire cohort, correlated strongly with PCS in patients with tetraplegia and did not correlate with PCS in patients with paraplegia. Mobility subgroup and individual items scores showed the strongest correlations with the PCS in the entire cohort as well as in patients with tetraplegia, followed by self-care and sphincter management. Correlations between SCIM scores and MCS for all patients were negligible. Conclusion: This is the first study to objectively evaluate the relative importance of specific functional abilities in the HRQoL in tSCI patients. This work is significant because it determines which specific functional abilities are mostly related to HRQoL, and highlights the differences between patients with tetraplegia and paraplegia. Our findings could help clinicians to guide the treatment and rehabilitation plans based on the importance of specific functional abilities in relation to the HRQoL.

\section{3}

Comparing the accuracy of logistic regression and artificial intelligence models to predict ambulatory outcomes after traumatic spinal cord injury in AIS (ASIA Impairment Scale) B and C: a prospective study from a Canadian multicentre spinal cord injury registry. Philippe Phan, ${ }^{1,2,3}$ Qiong Zhang, ${ }^{4,5}$ Nader Fallah, ${ }^{4,5}$ Carly S. Rivers, ${ }^{4}$ Vanessa K. Noonan, ${ }^{4,5}$ Tova Plashkes, ${ }^{4}$ Eugene K. Wai, ${ }^{2,3}$ Darren M. Roffey, ${ }^{1,3}$ Eve C. Tsai, ${ }^{1,3,6}$ Férôme Paquet, ${ }^{7}$ the RHSCIR Network. From the ${ }^{1}$ Ottawa Combined Adult Spinal Surgery Program, The Ottawa Hospital, Ottawa, Ont.; ${ }^{2}$ Division of Orthopaedic Surgery, Department of Surgery, Faculty of Medicine, University of Ottawa, The Ottawa Hospital, Ottawa, Ont.; ${ }^{3}$ Clinical Epidemiology Program, Ottawa Hospital Research Institute, Ottawa, Ont.; ${ }^{4}$ Rick Hansen Institute, Blusson Spinal Cord Centre, Vancouver, BC; ${ }^{5}$ The University of British Columbia, Vancouver, BC; ${ }^{6}$ Division of Neurosurgery, Department of Surgery, Faculty of Medicine, University of Ottawa, The Ottawa Hospital, Ottawa, Ont.; ${ }^{7}$ Département Sciences Neurologiques, Pavillon Enfant-Jésus, CHU de Québec, Que.

Background: Models for predicting recovery in patients affected with traumatic spinal cord injury (tSCI) were developed for patients with partial injury and uncertain prognosis - that is, American Spinal Injury Association (ASIA) Impairment Scale (AIS) B and C. Using logistic regression and other artificial intelligence algorithms, we evaluated the efficacy of several prognostic models and compared their ability to effectively predict recovery. Methods: In a prospective cohort study using the Rick Hansen Spinal Cord Injury Registry (RHSCIR), we identified patients that sustained tSCI between 2004 and 2016. A total of 5 prognostic models (3 logistic regression [LR] and 2 artificial intelligence) were evaluated for their ability to predict walking ability 1 -year postinjury. Models were compared by means of area under the 
receiver operating characteristic curve (AUC). Results: A total of 675 tSCI patients within the RHSCIR were identified for our study, comprising 210 AIS A, 51 AIS B, 109 AIS C, and 305 AIS D patients. Our novel, 7-variable, modified LR model demonstrated an AUC of 0.854 (95\% confidence interval [CI] $0.808-$ 0.900). For the same cohort, van Middendorp and colleagues' LR model and Hick and colleagues' simplified LR model generated AUCs of 0.833 (95\% CI 0.771-0.895) and 0.821 (95\% CI $0.754-$ 0.887 ), respectively, whereas the artificial intelligence model (using neural networks) generated an AUC of 0.872 . There were no significant differences in predictive accuracy between models $(p<0.05)$. Predictive accuracies (AUCs) for the remaining 515 AIS A and D patients using van Middendorp and colleagues' and Hicks and colleagues' models were 0.954 and 0.949 , respectively. Conclusion: Application of a novel, 7-variable LR model, 2 previously tested multivariate LR models, and 2 artificial intelligence models demonstrated limited predictive accuracy for AIS B and C patients. These findings suggest that former models garnered prognostic success by means of a disproportionate number of tSCI patients being AIS A or D, leading to increased predictive accuracy. Furthermore, no present model can predict with high accuracy ambulatory outcomes in AIS B and C patients, regardless of methodology. As such, this study suggests that future models may require integration of external variables (e.g., magnetic resonance imaging or relevant biomarkers) to increase prognostic ability in this subpopulation of tSCI patients.

\section{8}

Neurological recovery and functional outcomes in patients with acquired infections following acute traumatic spinal cord injury: pooled analysis using NACTN-STASCIS data. Fan Fiang, ${ }^{1}$ Blessing N.R. Jaja, ${ }^{1}$ Jefferson R. Wilson, ${ }^{2}$ Michael G. Feblings. ${ }^{1}$ From the ${ }^{1}$ Divisions of Neurosurgery, Orthopedic Surgery and Spinal Program, University of Toronto and Toronto Western Hospital, Toronto, Ont.; ${ }^{2}$ St. Michael's Hospital, Division of Neurosurgery, University of Toronto, Toronto, Ont.

Background: Acquired infections are common during hospital stays for traumatic spinal cord injury (SCI). This study investigated hospital-acquired pneumonia, wound infection, or sepsis impact potential for neurologic and functional recovery following SCI. Methods: Comparative analyses were conducted using pooled prospective data from the North American Clinical Trials Network (NACTN) SCI registry and the Surgical Timing in Acute Spinal Cord Injury Study (STASCIS) to evaluate the effect of hospital-acquired pneumonia, wound infection or sepsis (PWS) on American Spinal Injury Association (ASIA) Impairment Scale (AIS) grade improvement, respiratory function, and ambulation (assessed by the Spinal Cord Independence Measure [SCIM] 6 months after acute SCI $(n=1298)$. Penalized maximum likelihood regression analysis was performed with adjustment for relevant clinical confounders (baseline AIS grade, neurologic level of injury, and time to surgical decompression) and fixed effect of study. Results: Patients with PWS were more likely to be male $(22.8 \%$ v. $13.5 \%$; $p=0.007)$ and to present with a cervical- or thoracic-level neurologic injury that is complete (AIS grade A). They also were more likely to have surgical decompression within 24 hours of the injury $(26.0 \% \mathrm{v} .18 .8 \% ; p=$ $0.038)$ and to have had a longer hospital stay $(25$ v. $11 \mathrm{~d} ; p<$
0.001), compared with those without PWS. Relative to the latter group, patients with PWS were more likely to require assistance with breathing (odds ratio [OR] 4.72, 95\% confidence interval [CI $]$ 1.74-12.77) and require support with ambulation (OR 4.71, 95\% CI 1.93-1.48) 6 months postinjury, after accounting for confounders. There was no difference between groups with respect to AIS grade conversion at 6 months (OR 0.71, 95\% CI 0.39-1.30). Conclusion: The functional outcomes of SCI patients who experience acute in-hospital infections are considerably poorer than those of their counterparts without PWS, even though both have comparable neurologic recovery 6 months after the injury.

\section{3}

Interpreting neurologic recovery in acute spinal cord injury: Does the timing of the baseline neurologic examination influence outcome? Babak Sharifi, ${ }^{1}$ Carly S. Rivers, ${ }^{2}$ Nader Fallah, ${ }^{2,3}$ Zeina Wabeed, ${ }^{2}$ Vanessa K. Noonan, ${ }^{2,3}$ Tamir Ailon, ${ }^{4}$ Nicolas Dea, ${ }^{5}$ Scott Paquette, ${ }^{4}$ Fobn Street, ${ }^{5,6}$ Charles Fisher, ${ }^{4,6}$ Marcel Dvorak, ${ }^{4,6}$ Brian Kwon. ${ }^{4,6,7}$ From the ${ }^{1}$ Vancouver Acute - Adult Spine Fellow, Department of Orthopaedics, University of British Columbia, Vancouver, BC; ${ }^{2}$ Rick Hansen Institute, Blusson Spinal Cord Centre, Vancouver, BC; ${ }^{3}$ The University of British Columbia, Vancouver, BC; ${ }^{4}$ Department of Orthopaedics, University of British Columbia, Vancouver, BC; ${ }^{5} \mathrm{Com}-$ bined Neurosurgical and Orthopedic Spine Program, Vancouver General Hospital, University of British Columbia, Vancouver, BC; ${ }^{6}$ Vancouver Spine Surgery Institute, Vancouver, BC; ${ }^{7}$ International Collaboration on Repair Discoveries (ICORD), University of British Columbia, Vancouver, BC

Background: Predicting neurologic recovery after acute traumatic spinal cord injury (SCI) obviously becomes easier as time passes from the time of injury. For example, a surgeon who deems a patient to be an American Spinal Injury Association (ASIA) Impairment Scale (AIS) A complete tetraplegic 6 hours postinjury is undoubtedly less certain about the ultimate neurologic outcome than he or she is for a patient assessed as an AIS A tetraplegic 6 days or 6 months postinjury. But what if the neurologic assessment is done at 12 or 18 hours postinjury? Because acute SCI clinical trials often enrol patients within 12-24 hours of injury, the neurologic examination must be conducted very soon after injury. The objective of this study was to determine the relationship between the time of assessment (TOA) within the first 48 hours and the subsequent neurologic recovery. Methods: The Rick Hansen Spinal Cord Injury Registry (RHSCIR) was used to identify patients with acute cervical SCI who were admitted and examined within 48 hours of injury. The time of injury and the TOA to assign their baseline AIS grade were recorded. AIS grade conversion and motor score improvement were measured and related to the TOA. Results: In a preliminary analysis of 68 cervical AIS A SCI patients examined within 48 hours of injury, we found that AIS grade conversion occurred in 25 (36.8\%). Those with a TOA within 4 hours of injury showed a $54 \%$ conversion rate, whereas those with a TOA more than 12 hours postinjury had only a $25 \%$ conversion rate. Improvement in motor score 
in cervical AIS A patients with a TOA within 8 hours postinjury was much greater than in those with a TOA more than 8 hours after injury (16 v. 7.5 motor points, $p=0.039$ ). Conclusion: This is the first study to demonstrate that the TOA within the first 24-48 hours postinjury is an important parameter to consider when interpreting neurologic recovery after acute SCI. This is particularly important for nonrandomized clinical trials in acute SCI that enrol patients within this acute time frame.

\section{1}

Will cost transparency in the operating theatre cause surgeons to change their practice? R. Andrew Glennie, William Oxner, Sean Barry, Jacob Alant, Sean Cbristie.

Background: Cost-containment strategies continue to be emphasized in health care delivery, with a specific emphasis on surgical procedures. However, many surgeons do not have a thorough working knowledge regarding the costs of devices and surgical equipment, and their impact on overall costs. There are a variety of possible explanations for this, including rapid technological advancement and price insensitivity. The purpose of this study is to determine whether surgeons will change their choice of implants or equipment once they are aware of the associated specific costs. Methods: A thorough bottom-up case-costing method was used to determine the costs of all implants used by a spine surgical service at a large tertiary care centre. Costs were collected for an initial 5 -month period (blinded) during which surgeons were not aware of costs, followed by another 5 -month period (unblinded) during which detailed cost information was provided to the surgeons. Three procedures, anterior cervical discectomy and fusion (ACDF), posterior cervical fusion, and lumbar interbody fusions, were included. Statistical analysis was undertaken with STATA software. Results: Instrumentation costs decreased by a mean of $\$ 478$ once actual prices were known; however, this result was not statistically significant $(p=$ $0.069)$. Only ACDF procedures showed significant cost savings (mean $\$ 754, p=0.009$ ). Procedural costs were not significantly lower after blinding (\$5717.71 blinded v. $\$ 5420.58$ unblinded, $p=0.194$ ), nor were the total mean overall costs of admission (\$10 461.41 blinded v. $\$ 10862.26$ unblinded, $p=$ $0.228)$. There were no significant differences in individual surgeon costs or in health-related quality of life outcomes throughout the study. Conclusion: Although costs decrease for implants in surgery when prices are known, this appears to have little or no effect on overall costs of care. Length of stay and efficient use of operating room time appear to have greater effects on global costs. Future efforts to encourage efficient cost savings should focus on practice patterns for similar conditions rather than limiting the use of certain implants.

\section{7}

Perioperative anemia in minimally invasive compared with open posterior lumbar decompression and fusion for degenerative spinal stenosis. Tan Chen, ${ }^{1}$ Stephen Lewis, ${ }^{2,3}$ Raja Rampersaud. ${ }^{2,3}$ From the ${ }^{1}$ Orthopaedic Surgery, University of Toronto, Toronto, Ont.; ${ }^{2}$ Krembil Neuroscience Center, Toronto Western Hospital, University Health Network, Toronto, Ont.; ${ }^{3}$ Division of Orthopaedic Sur- gery, Toronto Western Hospital, University Health Network, Toronto, Ont.

Background: Recent efforts have seen a push in spine surgery to improve postoperative pain and health care efficiency with attention to reducing length of stay in hospital. Minimally invasive surgical (MIS) techniques in spinal decompression and fusion are relatively novel, with reported benefits of reduced perioperative anemia and transfusions, which reduces morbidity, mortality and rehabilitation. This study evaluates focal MIS posterior instrumented fusion against open techniques with regard to perioperative blood loss, transfusion requirements, length of stay, adverse effects, and in-hospital cost. Methods: Retrospective review was performed by use of prospective data from 80 patients (46 MIS; 34 open). Inclusion criteria were age more than 65 years, elective 1 - and 2-level posterior decompression and fusion for degenerative lumbar pathology. Demographics, perioperative adverse events, length of stay, hemoglobin level preoperatively and at discharge, and perioperative transfusions during stay were collected. In-hospital microcosting was collected from the Ontario Case Costing Initiative. Adverse events were captured using the Orthopaedic Surgical Adverse Event Severity System. Results: The MIS and open groups were comparable in age (MIS $70.7 \mathrm{yr}$; open $72.1 \mathrm{yr}$ ) and body mass index (MIS 28.8; open 28.7). In-hospital direct, indirect and total cost were comparable (MIS: \$14 704.45, \$4993.01 and \$19 697.46, respectively; open: \$16 504.46, \$4739.67 and \$21 244.07, respectively). Length of stay among the MIS group was significantly shorter (MIS $5.0 \mathrm{~d}$; open $9.9 \mathrm{~d}$; $p<0.01$ ). Although preoperative hemoglobin levels were comparable (MIS $132.9 \mathrm{~g} / \mathrm{L}$, open $131.3 \mathrm{~g} / \mathrm{L}$ ), absolute levels at discharge differed significantly (MIS $112.5 \mathrm{~g} / \mathrm{L}$; open $100.8 \mathrm{~g} / \mathrm{L} ; p<0.01$ ) as did the mean change in levels (MIS $20.3 \mathrm{~g} / \mathrm{L}$; open $30.9 \mathrm{~g} / \mathrm{L}$; $p<0.01$ ). Forty-seven percent of patients in the open group received transfusions postoperatively (mean 2.0 units packed red blood cells per patient). No MIS patients required transfusion. Incidence of adverse events was similar between groups (MIS $28.3 \%, 0.41$ events/patient; open $29.4 \%, 0.47$ events/patient; $p=0.79)$. Conclusion: Focal MIS posterior decompression and instrumented fusion in the lumbar spine significantly reduces perioperative anemia, need for transfusion, and length of hospitalization as compared with traditional open technique. Overall costs were similar, with more expensive MIS implants offset by shorter length of stay.

\section{7}

Epidemiology and outcomes of neck pain following surgery for cervical radiculopathy. Fred Xavier, ${ }^{1}$ Ayoub Dakson, ${ }^{1}$ Greg McIntosh, ${ }^{2}$ Sean Christie, ${ }^{1}$ CSORN Investigators. ${ }^{2}$ From the ${ }^{1}$ Department of Surgery (Spine), Dalhousie University, Halifax, NS; ${ }^{2}$ Canadian Spine Outcomes and Research Network, Toronto, Ont.

Background: Cervical degenerative disc disease affects $1 / 5$ of adults worldwide, often leading to symptomatic cervical radiculopathy. Several authors report improvement in arm pain following surgery; however, neck pain is generally not felt to improve. The purpose of this study was to determine whether surgery for cervical radiculopathy also leads to a significant improvement in neck pain. Methods: This retrospective 
cohort study used the Canadian Spine Outcomes and Research Network (CSORN) registry to identify all patients who received primary surgery for cervical radiculopathy secondary to degenerative causes. The cohort was divided into 2 groups (fusion and arthroplasty). The fusion cohort was further subdivided into 3 subgroups (1-level, 2-level, and > 2-level anterior cervical discectomy and fusion $[\mathrm{ADCF}])$. The primary outcome was achieving minimal clinically important difference (MCID) on the visual analogue scale (VAS) for neck pain. Secondary outcomes were based on achieving MCID for Neck Disability Index (NDI), VAS arm pain, short form 12 (SF-12, physical and mental component score [PCS; MCS]) and EuroQOL 5-dimension (EQ-5D). Patient-reported outcomes were obtained preoperatively and out to 1 year postoperatively using paired $t$ tests, Pearson $\chi^{2}$ test and unpaired $t$ tests. Differences in proportions of patients achieving MCID between different treatment groups were assessed using the $\chi^{2}$ analysis. Analysis of covariance (ANCOVA) and a logistic regression model were applied to determine any demographic predictors of the continuous and categorical variables, respectively. All levels of significance were 2 -tailed and set at $p<0.05$. Results: We identified 191 patients with cervical radiculopathy subgrouped into those treated with 1-level ACDF (46.1\%), 2-level ACDF (28.3\%), 3-level ACDF (9.9\%), cervical arthroplasty $(11.5 \%)$ and hybrid procedures (4.2\%). At 1-year follow-up, the overall mean VAS-neck pain and VAS-arm pain improved and achieved MCID for all the patients by a mean of 3.61 and 4.39 points, respectively. However, no difference in the thresholds for MCIDs for VAS-arm pain, VAS-neck pain, MCS, PCS and NDI were observed when comparing across the different surgical approaches $(p>0.05)$. Conclusion: Our results demonstrated a significant improvement in VAS-neck pain 1 year after cervical spine surgery, regardless of the technique used. This study provides new information on the improvement in chronic neck pain following surgery for cervical radiculopathy and may be useful in preoperative education.

\section{Poster Presentations}

30

Clinical presentation, management and outcomes of sacral metastases: a multicentre, prospective case series. Raphaële Charest-Morin, ${ }^{1}$ Nicolas Dea, ${ }^{2}$ Anne Versteeg, ${ }^{3}$ Arjun Saghal, ${ }^{4}$ Peter Pal Varga, ${ }^{5}$ James M. Schuster, ${ }^{6}$ Michael H. Weber ${ }^{7}$ Michelle 7. Clarke, ${ }^{8}$ Laurence D. Rbines, ${ }^{9}$ Stefano Boriani, ${ }^{10}$ Chetan Bettegowda, ${ }^{11}$ Michael G. Feblings, ${ }^{4}$ Paul M. Arnold, ${ }^{12}$ Ziya L. Gokaslan, ${ }^{13}$ Charles G. Fisher. ${ }^{2}$ From the ${ }^{1}$ Centre Hospitalier Universitaire de Québec, Québec, Que.; ${ }^{2}$ University of British Columbia and Vancouver General Hospital, Vancouver, BC; ${ }^{3}$ Utrecht, Netherlands; ${ }^{4}$ University of Toronto, Toronto, Ont.; ${ }^{5}$ National Center for Spinal Disorders and Buda Health Center, Budapest, Hungary; ${ }^{6}$ Perelman School of Medicine, Philadelphia, Penn.; ${ }^{7}$ McGill University, Montreal, Que.; ${ }^{8}$ Mayo Clinic, Rochester, NY; ${ }^{9} \mathrm{MD}$ Anderson Cancer Center, The University of Texas, Houston, Tex.; ${ }^{10}$ IRCCS Istituto Orthopedico Galeazzi, Milan, Italy; ${ }^{11}$ Johns Hopkins University School of Medicine, Baltimore, Md.; ${ }^{12}$ The University of Kansas Medical Center, Kansas City, Kans.; ${ }^{13}$ Rhode Island Hospi- tal, The Warren Alpert Medical School of Brown University, Providence, RI.

Background: Sacral metastases are rare, and literature regarding their management is sparse. This is a descriptive case series that reports on health-related quality of life (HRQOL) and pain in patients treated for sacral metastases with surgery or radiotherapy (RT). The secondary objectives were to describe the adverse event (AE) profile and change in neurologic function in this population. Methods: Patients presenting with sacral metastases were identified from the Epidemiology, Process and Outcomes of Spine Oncology (EPOSO) data set, a prospective, multicentre observational study. Patients requiring surgery or RT between August 2013 and February 2017 were prospectively enrolled. HRQOL, assessed by the Spine Oncology Study Group Outcomes Questionnaire (SOSGOQv2.0), the Short Form-36 version 2 (SF-36v2), and the EuroQol-5 dimension (EQ-5D) was documented at baseline and at 6 weeks, 3 months and 6 months post-treatment. Pain numeric rating scale (NRS), AEs, lower extremities motor score (American Spinal Injury Association [ASIA]), and bowel and bladder function were recorded at baseline and follow-up. Results: We identified 23 patients with sacral metastases: 8 patients underwent surgery with or without RT and 15 patients underwent RT alone. Mean age was 59.3 years (standard deviation [SD] 11.7), and 13 patients were female. At 6 months, 3 (37.5\%) surgical patients and $2(13.3 \%)$ RT patients were deceased. There was a trend showing that surgical patients had worse baseline HRQOL and pain. Pain NRS, EQ-5D, SOSGOQv2.0, and the mental component of the SF-36v2 showed improvement, irrespective of treatment. The variation in the outcome scores observed between visits within the same group were not statistically different $(p>0.05)$. Ten AEs occurred in the surgical cohort, dominated by wound complications $(n=$ 4). Bowel and bladder function improved at 6 weeks in both groups. The lower extremity mean motor score marginally improved at 3 months. Conclusion: Surgical treatment and RT are valid complementary alternatives for symptomatic sacral metastases. Improvement in HRQOL can be expected with an acceptable $\mathrm{AE}$ rate.

98

Impact of goal-directed fluid therapy on postoperative outcomes after spine and orthopedic surgery: a systematic review and meta-analysis. Rakan Bokbari, ${ }^{1,2}$ Eunice Linb You, ${ }^{2}$ Oliver Lasry, ${ }^{2}$ Gabriele Baldini, ${ }^{3}$ Michael Weber. ${ }^{4,5}$ From the ${ }^{1}$ Division of Neurosurgery, King Abdulzaziz University, Jeddah, Saudi Arabia; ${ }^{2}$ McGill University, Montréal, Que.; ${ }^{3}$ Department of Anesthesia, Montreal General Hospital, Montréal, Que.; ${ }^{4}$ Orthopa, Montréal, Que.; ${ }^{5}$ Department of Orthopaedic Surgery, Montreal General Hospital, Montréal, Que.

Background: Goal-directed fluid therapy (GDFT) is a perioperative fluid management strategy that tailors resuscitation based on the objective measures of hypovolemia and fluid responsiveness, with the ultimate goal of optimizing cardiac output and achieving adequate tissue perfusion while avoiding excessive fluid restriction or overload. It has been shown to improve patient outcomes in high-risk patients undergoing cardiac and noncardiac surgeries. However, its role in orthopedic subspecialties, 
including spine surgery, remains poorly studied. We therefore systematically review the orthopedic literature with the purpose of presenting what is known about the effect of GDFT after spine and orthopedic surgery. Methods: We systematically searched MEDLINE and Embase were systematically searched for randomized trials and nonrandomized cohort studies that compared outcomes between patients treated with GDFT or conventional fluid management (control group). Patients who received fluid therapy based on physiologic parameters other than urine output, blood pressure and heart rate in the context of an intraoperative protocol were included. Results: Of the 6783 articles retrieved, 11 studies that enrolled a total of 992 patients $(n=$ 493 GDFT, $n=499$ control) were included. Of these studies, only 2 involved patients undergoing spine surgery. Goal-directed fluid therapy was associated with a significant decrease in overall complications (odds ratio [OR] $0.52,95 \%$ confidence interval [CI] 0.36-0.75). There was no effect on mortality, length of stay or any organ-specific complication, with the exception of a lower incidence of kidney dysfunction (OR $0.47,95 \%$ CI $0.25-0.89$ ) and gastrointestinal complications (OR $0.49,95 \%$ CI $0.27-0.90$ ). These results were mainly driven by outcomes reported in studies conducted in patients undergoing nonspine surgery. Conclusion: Goal-directed fluid therapy in spine surgery is understudied despite showing promise in the orthopedic population. This systematic review provides rationale for further determining the impact of GDFT on postoperative outcomes after major spine surgery. A randomized clinical trial is currently underway at our centre.

\section{5}

Traumatic cauda equina injury in Canada: What predicts neurological and bowel or bladder sphincter outcomes? Najmedden Attabib, ${ }^{1}$ Colleen O'Connell, ${ }^{2}$ Dilnur Kurban, ${ }^{3}$ Carly S. Rivers, ${ }^{3}$ Chris Bailey, ${ }^{4}$ Sean Cbristie, ${ }^{5}$ Karen Ethans, ${ }^{6}$ Heather Flett, ${ }^{7}$ Zulio Furlan, ${ }^{8}$ Eve Tsai, $, 10,11$ RHSCIR Network, Amanda Carson. ${ }^{1}$ From the ${ }^{1}$ Dalhousie University, Saint John Regional Hospital, St. John, NB; ${ }^{2}$ Physical Medicine \& Rehabilitation, Dalhousie University Faculty of Medicine, Halifax, NS; ${ }^{3}$ Rick Hansen Institute, Blusson Spinal Cord Centre, Vancouver, BC; ${ }^{4}$ Division of Orthopaedics, Department of Surgery, Schulich School of Medicine and Dentistry, University of Western Ontario, London, Ont.; ${ }^{5}$ Research Division of Neurosurgery, Dalhousie University, Halifax, NS; 'University of Manitoba, Department of Medicine, Winnipeg, Man.; ${ }^{7}$ Department of Psychology, University of Toronto, Toronto, Ont.; ${ }^{8}$ Comprehensive Integrated Pain Program, Toronto Western University, Toronto, Ont.; ${ }^{9}$ Ottawa Combined Adult Spinal Surgery Program, The Ottawa Hospital, Ottawa, Ont; ${ }^{10}$ Clinical Epidemiology Program, Ottawa Hospital Research Institute, Ottawa, Ont.; ${ }^{11}$ Division of Neurosurgery, Department of Surgery, Faculty of Medicine, University of Ottawa, The Ottawa Hospital, Ottawa, Ont.

Background: Traumatic cauda equina injury (TCEI), usually resulting from injury to L1 and below, results in deleterious independence impairments such as bowel or bladder sphincter dysfunction and sensory or motor weaknesses. Our objective was to determine what factors predict neurologic and functional (urinary, bowel) recovery to aid in prognosis and optimal man- agement. Methods: The analysis cohort consisted of patients with acute TCEI participating in the Rick Hansen Spinal Cord Injury Registry (RHSCIR). Lower extremity motor score (LEMS), neurologic level of injury and voluntary anal contraction (VAC) were determined from the admission and discharge International Standards for Neurological Classification of Spinal Cord Injury (ISNCSCI) examination. Change in urinary or bowel function was assessed by the Functional or Spinal Cord Independence Measure (FIM or SCIM) at discharge and follow-up. Age, sex, mechanism of injury, comorbidities, surgical management and factors such as length of stay (LOS) were examined. Results: The analysis cohort included 217 participants, of whom $77.0 \%$ were male, $49.1 \%$ had fall-related injuries and $47.5 \%$ had L1 neurology level; the mean age of participants was $40.0 \pm 17.2$ years; $97.4 \%$ of the participants underwent surgery and median time from injury to first surgery was 26.5 hours. The median time from injury to fist RHSCIR acute and rehabilitation sites were 9 hours and 24 days, respectively. The participants stayed a median of 19 and 58 days, respectively, at acute and rehabilitation facilities. The median change in LEMS and in bladder and bowel functions from first admission to last discharge were 4,4 and 3, respectively. About $30.6 \%$ of participants with no anal contraction at their first admission converted to "yes" at their final discharge. Preliminary multivariate analysis indicated age, sex and rehabilitation LOS were significant predictors of bladder function recovery; rehabilitation LOS was the only significant predictor of bowel function recovery. Age and surgery status affected the change in LEMS, but they did not reach statistical significance. Conclusion: No significant predictor was identified for neurologic recovery in TCEI patients; age, sex and LOS were significant predictors of bladder and bowel function recovery.

\section{1}

Use of spinal intraoperative 3-dimensional navigation by Canadian surgeons and trainees: a population-based time trend study. Daipayan Guba, Ali Mogbaddamjou, Zaneen Fiwani, Naif Alotaibi, Albert Yee, Victor Yang. From the Sunnybrook Research Centre, Toronto, Ont.

Background: Computer-assisted navigation (CAN) is a useful adjunct to improve the accuracy of spinal instrumentation and for trainee education, but is not widely adopted by spinal surgeons partly because of lack of training, high capital costs and workflow hindrances. Here, we characterize the spatiotemporal use of spinal CAN in a single-payer health care system, such as in Canada, and assess the impact of intraoperative CAN on trainee proficiency. Methods: A prospectively maintained Ontario database of patients undergoing spinal instrumentation from 2005 to 2014 was reviewed retrospectively. Data were collected on treated pathology, spine region, surgical approach, institution type, surgeon specialty, the use of 2-dimensional or 3 -dimensional CAN and revision surgeries within 2 years of the index procedure. Predictors of CAN usage and revision surgery were identified. Trainee comfort with CAN and its impact on technical proficiency were assessed using an electronic questionnaire distributed to all Canadian orthopedic surgical and neurosurgical trainees across 15 nationwide training programs. Results: A total of $16.8 \%$ of instrumented fusions in our provincial cohort were $\mathrm{CAN}$-guided, predominantly by 3 -dimensional 
CAN. Navigation was employed more frequently in academic institutions $(15.9 \%$ v. $12.3 \%, p<0.001)$ and by neurosurgeons more than orthopedic surgeons $(21.0 \%$ v. $12.4 \%, p<0.001)$. Revision surgery was required in $6.4 \%$ of patients undergoing instrumented fusion, more frequently for trauma and deformity cases, for cases performed at academic centres and for cases performed without CAN guidance. A total of $34.1 \%$ of residents reported being fully comfortable in the setup and use of spinal CAN, greater for neurosurgical than for orthopedic surgical trainees $(48.1 \%$ v. $11.8 \%, p=0.008)$. The use of CAN for thoracic instrumentation increased the mean self-reported proficiency rank across all trainees by $11.0 \%(p=0.036)$, with orthopedic residents also reporting an increase in mean proficiency rank of $18.0 \%$ for atlantoaxial instrumentation ( $p=$ 0.014 ) with CAN guidance. Conclusion: In current practice, spinal CAN is employed most frequently by neurosurgeons and in academic centres. The use of CAN is associated with a significant decrease in associated revision surgeries. Most spine surgical trainees are not fully comfortable with the setup and use of intraoperative CAN, but do report an increase in technical comfort with CAN guidance, particularly for thoracic instrumentation. Increased education in spinal CAN starting at the trainee level, particularly for orthopedic surgery, may improve adoption.

\section{4}

Indications and outcomes of cell saver in adolescent scoliosis correction surgery: a systematic review. Vandit Sardana, Nicholas Stone, Paul Missiuna. From the Division of Orthopedics, McMaster University, Hamilton, Ont.

Background: Several blood conservation strategies exist to minimize the consequences of blood loss because of scoliosis correction surgery. The utility of intraoperative cell salvage has been contested in the literature with respect to benefits and cost. High-quality randomized control trials are needed to help surgeons make an informed decision about including Cell Saver into their practice. The aim of this study was to determine the indications and outcomes for intraoperative cell salvage during adolescent scoliosis correction surgery. Methods: We performed a systematic review of all available evidence. Results: There were 7 eligible studies that directly compared the use of Cell Saver (349 patients) against a control (non-Cell Saver) group (244 patients). Most of the demographic and hematologic data were consistent between both groups. The mean volume of blood that was re-infused into patients in the Cell Saver group was $453.8 \mathrm{~mL}$. Patients in the Cell Saver group received significantly fewer allogenic blood transfusions than the did patients in the control group, with $32.6 \%$ less intraoperatively, $45.9 \%$ less postoperatively, and $47.3 \%$ less perioperatively. On average, Cell Saver patients received 1.0 fewer units of allogenic blood compared with control patients. Conclusion: Cell Saver reduces the demand for allogenic transfusion. This review supports the use of Cell Saver if the associated monetary costs are expected to be less than the cost of transfusing 1 unit of allogenic blood.

\section{5}

Elective lumbar laminectomies: preoperative investigations, transfusion rate and postoperative outcomes. Mina
Tobidi, Tiffany Lung, David Yen. From the Queen's University, Kingston, Ont.

Background: The primary purpose of this study was to determine the prevalence and types of preoperative blood work for patients undergoing elective lumbar laminectomy for spinal stenosis. Secondary objectives were to determine the prevalence of intraoperative tranexamic acid administration, intraoperative complications, hospital length of stay and 30-day readmission in this population, and to determine whether outcomes varied by surgical approach. Methods: A retrospective electronic chart review was conducted on all patients 18 years of age and older who underwent laminectomy at 1 or more levels of the lumbar spine by an orthopedic spine surgeon (DY) between July 1, 2013, and June 30, 2017. All procedures were performed at the university-affiliated inpatient hospital or ambulatory care hospital. Results: A total of 256 patients underwent elective lumbar laminectomy of 1 or more levels by a single orthopedic spine surgeon during the study period. Among these patients, $89 \%$ underwent at least 1 preoperative blood test, and 31\% underwent preoperative blood type and screen. The intraoperative complication rate, which included dural tears that required surgical repair and intraoperative blood transfusion, was $2.34 \%$. Less than $2 \%$ of patients received intravenous tranexamic acid intraoperatively. In the 30 days following surgery, there were no hospital readmissions related to complications. Length of stay was lower for patients treated with bilateral decompression using a unilateral approach, compared with the traditional bilateral approach $(p=$ $0.0063)$. Estimated blood loss and duration of surgery did not differ significantly between the 2 groups $(p=0.86$ and $p=0.30$, respectively). Conclusion: Hospital policies should be reevaluated to address the overuse of unnecessary preoperative investigations for elective lumbar laminectomies, which have low perioperative transfusion and complication rates. Bilateral decompression using a unilateral approach is associated with shorter length of stay in hospital without increased operative time or risk of complications. Further research is warranted to determine if these results are reproducible after controlling for severity of spinal stenosis.

49

Treatment of mild cervical myelopathy: What factors are associated with the decision for surgical intervention? Michael Bond, ${ }^{1}$ Hanbing Zhou, ${ }^{1}$ Bradley Facbos, ${ }^{2}$ Michael Jobnson, ${ }^{3}$ Charles Fisher, ${ }^{1}$ Chris Bailey, ${ }^{4}$ Sean Christie, ${ }^{5}$ Raphaele Charest-Morin, ${ }^{6}$ Andrew Nataraj, ${ }^{7}$ Neil Manson, ${ }^{8}$ Hamilton Hall, ${ }^{9}$ Ken Thomas, ${ }^{10}$ Raja Rampersaud, ${ }^{11}$ Greg McIntosh, ${ }^{12}$ Nicolas Dea. ${ }^{1}$ From the ${ }^{1}$ Combined Neurosurgical and Orthopaedic Spine Program, Vancouver General Hospital and University of British Columbia, Vancouver, BC; ${ }^{2}$ Department of Clinical Neurosciences, Faculty of Medicine, University of Calgary, Calgary, Alta.; ${ }^{3}$ Department of Surgery, Section of Orthopedics and Neurosurgery, University of Manitoba, Winnipeg, Man.; ${ }^{4}$ Department of Surgery, Division of Orthopaedics, London Health Science Centre, Western University, London, Ont.; ${ }^{5}$ Division of Neurosurgery, Halifax Infirmary, Halifax, NS; ${ }^{6}$ Department of Orthopaedics, Centre Hospitalier Universitaire de Québec, Quebec, Que.; ${ }^{7}$ University of Alberta Complex Spine Surgery Program, Edmonton, Alta.; 
${ }^{8}$ Canada East Spine, Department of Surgery, Dalhousie University, St. John, NB; ' ${ }^{9}$ Department of Surgery University of Toronto, Toronto, Ont.; ${ }^{10}$ Department of Surgery, Department of Clinical Neurosciences, University of Calgary, Calgary, Alta.; ${ }^{11}$ Division of Orthopaedic Surgery, Toronto Western Hospital, Toronto, Ont.; ${ }^{12}$ Canadian Spine Society Research Operations, Markdale, Ont.

Background: Surgical treatment of cervical myelopathy is associated with improved outcomes regardless of disease severity; however, a trial of supervised rehabilitation is recommended as an alternative to surgical treatment for patients with mild cervical myelopathy (modified Japanese Orthopaedic Association [mJOA] scale score $\geq 15$ ). The purpose of this study was to evaluate what factors are associated with selection for surgical intervention in patients with mild cervical myelopathy. Methods: Patients with cervical myelopathy from 7 Canadian centres were prospectively enrolled in the Canadian Spine Outcomes and Research Network (CSORN) database. Demographic, clinical, radiographic and health-related quality of life (HRQOL) data were collected on all patients for baseline data. Multivariable logistic regression modelling was performed to identify predictors of surgical intervention. Results: We enrolled 360 patients, of whom 101 were categorized as having mild myelopathy; $83(82.2 \%)$ were treated surgically and $18(17.8 \%)$ were treated conservatively. Mean age was 56 years, and $63(62.4 \%)$ patients were male. There were no differences between surgical and nonsurgical patients with respect to body mass index, mJOA or age $(p>0.05)$. Patients who underwent surgery had higher baseline pain $(5.18 \mathrm{v} .4 .53)$ and higher disability (Neck Disability Index [NDI]) (35.03 v. 33.87) scores, which did not reach statistical significance. There was no difference between groups for any radiographic parameters including spinal cord compression, spine alignment and cord signal change $(p>0.05)$. Multivariable analysis showed that patients selected for surgical intervention had less than 1 year of symptom duration, higher neck pain scores, lower quality of life scores (EuroQOL 5 -dimension [EQ-5D]) and higher disability (NDI) scores $(p<$ 0.05). Conclusion: Patients who were treated surgically for mild cervical myelopathy did not differ from those treated nonoperatively with respect to demographic or radiographic parameters. Patients were more likely to have surgical intervention if duration of symptoms was shorter and they had worse pain and quality of life scores.

83

Mild degenerative cervical myelopathy: moving beyond the modified Japanese Orthopaedic Association score to identify surgical candidates. Fetan H. Badbiwala, ${ }^{1}$ Markus Schomacher, ${ }^{2}$ Christopher D. Witiw, ${ }^{1}$ Farshad Nassiri, ${ }^{1}$ Mubammad A. Akbar, ${ }^{1}$ Zamir Merali, ${ }^{1}$ Alireza Mansouri, ${ }^{1}$ Fefferson R. Wilson, ${ }^{1}$ Michael G. Feblings. ${ }^{1}$ From the ${ }^{1}$ Division of Neurosurgery, Department of Surgery, University of Toronto, Toronto, Ont.; ${ }^{2}$ Vivantes Klinikum Neukölln, Berlin, Germany.

Background: The value of surgery in the context of mild degenerative cervical myelopathy (DCM) is unclear. There is thought that mild DCM may represent a heterogeneous population. We sought to dissociate phenotypes of mild DCM patients associated with degree of impairment in quality of life (QOL) and surgical outcomes. Methods: Patients with mild DCM (modified Japanese Orthopaedic Association [mJOA] 15-17) enrolled in the prospective multi-entre AOSpine Cervical Spondylotic Myelopathy North America or International (CSM-NA, CSM-I) studies were identified. A $k$-means clustering algorithm was applied to baseline QOL (short-form 35 [SF-36]) scores to separate patients into 2 groups. Baseline variables, including demographic and treatment characteristics, were compared between clusters to explore phenotypes associated with each cluster. Surgical outcomes, defined by change in SF-36 scores at 1 year, were compared between clusters. A $k$-nearest neighbours $(\mathrm{kNN})$ algorithm was then used to evaluate the ability to classify patients into the 2 clusters by baseline clinical variables alone. Results: A total of 185 patients were included. Two groups were generated by $k$-means clustering. Cluster 1 had a greater proportion of female patients $(p=0.029)$, hyperreflexia $(p=0.036)$ and symptoms of gait difficulty $(p=0.025)$ or weakness $(p=0.041)$. Although baseline mJOA correlated with neither baseline QOL nor outcomes, cluster grouping had strong associations with both. The 2 clusters did not differ with respect to $\mathrm{mJOA}(p=0.28)$. However, cluster 1 was associated with significantly greater baseline disability rating on the Neck Disability Index [NDI] $(p<0.01)$ and impairment in QOL in nearly all domains of the SF-36 $(p<0.05)$. Likewise, patients in cluster 1 experienced significantly greater improvement in NDI $(p<0.01)$ and nearly all domains of the SF-36 $(p<$ 0.05 ) following surgery. A $\mathrm{kNN}$ algorithm could predict cluster classification with $67.5 \%$ (95\% confidence interval $63.8 \%$ $71.3 \%$ ) accuracy by sex and motor symptoms alone. Conclusion: Using an unsupervised machine-learning technique, we dissociated a clustering of patients within the mild DCM population that was strongly associated with baseline QOL and surgical outcomes. This clustering represented a distinct phenotype that could be identified from baseline clinical variables alone, and received heavy contributions from sex and motor symptoms.

\section{7}

Consultation and surgical wait times in patients with cervical spondylotic myelopathy: a prospective Canadian Spine Outcomes and Research Network study. Babak Sharifi, ${ }^{1}$ Charles Fisher, ${ }^{1}$ Brad Facobs, ${ }^{2}$ Mike Fohnson, ${ }^{3}$ Chris Bailey, ${ }^{4}$ Sean Christie, ${ }^{5}$ Raphaële Charest Morin ${ }^{6}$ Jerome Paquet, ${ }^{6}$ Andrew Nataraj, ${ }^{7}$ David Cadotte, ${ }^{2}$ Neil Manson, ${ }^{8}$ Hamilton Hall, ${ }^{9}$ Ken Thomas, ${ }^{2}$ Raj Rampersaud, ${ }^{9}$ Greg McIntosh, ${ }^{10}$ Nicolas Dea. ${ }^{1}$ From the ${ }^{1}$ University of British Columbia, Vancouver, BC; ${ }^{2}$ University of Calgary, Calgary, Alta.; ${ }^{3}$ University of Manitoba, Winnipeg, Man.; ${ }^{4}$ University of Western Ontario, London, Ont.; ${ }^{5}$ Dalhousie University, Halifax, NS; ' ${ }^{6}$ niversité Laval, Québec, Que.; ${ }^{7}$ University of Alberta, Edmonton, Alta.; 8Dalhousie University of Medicine, Saint John, NB; 9University of Toronto, Toronto, Ont; ${ }^{10}$ Canadian Spine Outcomes and Research Network, Toronto, Ont.

Background: Cervical spondylotic myelopathy (CSM) is the leading cause of spinal cord impairment. Prompt surgical intervention halts clinical deterioration and results in clinical improvement in most patients; however, in a public health care system, wait times to see a spine specialist and eventually access surgical treatment may be substantial. The goals of this study were to assess the consultation wait time (CWT) and surgical wait time 
(SWT), and to identify predictors of wait time length in this population. Methods: Consecutive patients enrolled in the Canadian Spine Outcomes and Research Network (CSORN) prospective, observational CSM study from March 2015 to July 2017 were included. Predictors of CWT and SWT were assessed using survival analysis. Results were stratified by disease severity based on modified Japanese Orthopaedic Association (mJOA) score. A data-splitting technique was used to develop and test the multivariable models looking at potential predictive factors. Results: A CSORN query returned $264 \mathrm{CSM}$ patients for CWT (88 women and 176 men). Mean age was 59.4 year. The median CWT was 46 days. There were $31 \%$ mild, 35/\% moderate and $33 \%$ severe cases of CSM. There was a statistically significant difference in median CWT between moderate and severe groups. There were 207 patients who underwent surgical treatment. Median SWT was 42 days. There was a significant difference between mild/ moderate and severe groups. Short symptom duration, less pain, radiologic cord compression, lower body mass index and lower physical component scores were predictive of shorter CWT. However, SWT was mainly affected by pain and symptom duration. Both CWT and SWT were shorter for CSM patients compared with lumbar stenosis patients $(p<0.001)$. Conclusion: Patients with CSM with more severe symptoms had shorter CWTs and SWTs. Patients with short symptom duration, less pain and spinal cord compression waited less to see a spine specialist in Canada. Patients with less pain and symptom duration less than a year had shorter SWTs.

\section{0}

Comparing the effect of arthroplasty versus fusion surgery on overall health status in patients with degenerative disc disease. Kim Vu, ${ }^{1}$ Stephen Kingswell, ${ }^{1}$ Philippe Pban, ${ }^{1}$ Alexandra Stratton, ${ }^{1}$ Darren Roffey, ${ }^{1}$ Mobammed Hoda, ${ }^{1}$ Eugene Wai, ${ }^{1}$ Peter Farzem, ${ }^{2}$ Facques Bouchard. ${ }^{3}$ From the ${ }^{1}$ The Ottawa Hospital, Ottawa, Ont.; ${ }^{2}$ McGill University, Montreal, Que.; ${ }^{3}$ University of Calgary, Calgary, Alta.

Background: Spinal fusion surgery is the traditional approach taken to treat pain resulting from degenerative disc disease (DDD). Artificial disc replacement (ADR) is a newer motionsparing surgical treatment option for DDD. However, there is little Canadian evidence available regarding the benefits of one treatment over the other. Our objective was to determine if outcomes of ADR differed compared with spinal fusion surgery. Methods: This study was a retrospective multivariate analysis of the prospectively collected data in the national Canadian Spine Outcomes and Research Network (CSORN) registry. Multivariate logistic regression was used to adjust for possible confounding effects of age, sex, levels operated on, baseline function and American Society of Anesthesiologists (ASA) score. All patients who underwent spinal surgery with a primary clinical complaint of back pain and primary radiological diagnosis of DDD were included. Results: There were 165 patients identified for the study: 75 had ADR in 1 or more spinal levels, and 90 had fusion surgery. Both ADR and fusion patients had similar physical function and mental health scores at baseline, but ADR patients reported a lower overall health status, as measured by EuroQoL visual analogue scale (EQ VAS). In adjusted multivariate analysis, ADR patients had significantly shorter surgery time (mean $1.8 \mathrm{v}$. $3.0 \mathrm{~d}, p=0.0150)$ and lower perioperative adverse events $(4.1 \% \mathrm{v}$.
$18.5 \%, p=0.0009)$. At 12 months' follow-up, overall improvement in EQ VAS for ADR patients was significantly higher (mean 20.3 v. $12.9, p=0.0489$ ), but there were no differences reported in any other outcomes. Conclusion: After adjusting for confounding, we demonstrated in a Canadian national database that patients with ADR had a higher improvement in overall health status compared with patients with spinal fusion surgery at 12 months, although they reported similar functional levels. Given the observational nature of this study, caution should be used in interpreting these results. Further study is required to determine if $\mathrm{ADR}$ is a superior treatment to spinal fusion surgery.

\section{1}

Validation of a finite element model to evaluate the stability of constructs used in the treatment of atlantoaxial instability. Timothy Lasswell, ${ }^{1}$ Duane Cronin, ${ }^{1}$ Fohn Medley, ${ }^{1}$ Fack Callagban, ${ }^{1}$ Colin McKinnon, ${ }^{1}$ Supriya Singh, ${ }^{2}$ Parbam Rasoulinejad. ${ }^{2}$ From the ${ }^{1}$ University of Waterloo, Waterloo, Ont.; ${ }^{2}$ Victoria Hospital, London Health Sciences Centre, London, Ont.

Background: Our objective was to validate a finite element (FE) model of the upper cervical spine for use as a tool in designing novel fusion constructs for the surgical treatment of atlantoaxial instability (AAI). Methods: An existing detailed neck FE model, previously validated under physiologic range of motion (ROM), was used to simulate AAI by implementing a type II odontoid fracture. A simplified Harms construct, consisting of an arrangement of 2-dimensional beam elements, was then added to the model. The model was run under flexion/extension, lateral bending and axial rotation, and construct stability was assessed by reporting C1/-C2 ROM at a load of $1.5 \mathrm{Nm}$. To validate the FE model, experimental testing was completed on 8 human cadaveric skull-C3 spine specimens. After implanting the Harms construct, a spinal loading simulator was used to test each cadaveric specimen, and construct stability was assessed by reporting C1-C2 ROM at a load of $1.5 \mathrm{Nm}$. Results: Simulating AAI in the FE model led to an increase in ROM for all motions that was within the scatter of experimental data from the literature. Addition of the simplified Harms construct into the FE model greatly reduced ROM compared with the destabilized AAI state. In axial rotation, the $\mathrm{FE}$ model predicted a $\mathrm{ROM}$ of $0.35^{\circ}$, which was within 2 standard deviations (SDs) of the experimental data (mean $0.9^{\circ}$, SD $0.4^{\circ}$ ). In lateral bending, the $\mathrm{FE}$ model predicted a ROM of $1.8^{\circ}$, which was outside 3 SDs of the experimental data (mean $0.7^{\circ}$, SD $0.1^{\circ}$ ), but still greatly reduced from the destabilized state (mean $5.6^{\circ}, \mathrm{SD} 1.4^{\circ}$ ). In flexion/extension, the $\mathrm{FE}$ model predicted a ROM of $2.0^{\circ}$ which was within $1 \mathrm{SD}$ of the experimental data (mean $2.1^{\circ}$, SD $1.1^{\circ}$ ). Conclusion: Finite element model simulations of AAI and a simplified Harms construct have been validated against experimental data. Future use of this model will focus on using it as a design tool to reliably predict the stabilizing effect of existing and novel fusion constructs for treating AAI.

\section{6}

Metal shedding and anterior cervical discectomy and fusion procedure using tantalum cages: a review of 117 levels fused. Melissa Nadeau, ${ }^{1}$ Fan Splawinski. ${ }^{2}$ From the ${ }^{1}$ Division of Spine, Department of Orthopaedics, University of 
British Columbia, Vancouver, BC; ${ }^{2}$ Vernon Jubilee Hospital, Vernon, BC

Background: Our primary objective was to assess fusion rate and clinical outcomes following anterior cervical discectomy fusion (ACDF) using a trabecular metal (TM) cage without bone graft. Our secondary objective was to correlate implant shedding to clinical and radiographic outcomes. Methods: We identified 126 consecutive patients from a single site undergoing ACDF with TM cages between 2006 and 2012. Operative details were obtained from patient charts, and clinical outcomes data were collected prospectively. Routine postoperative radiographs were assessed for fusions, radiolucency around the implant, implant subsidence, migration, fracture, shedding and screw back out. Clinical outcome measures included the Neck Disability Index (NDI), neck and arm pain severity by visual analog score (VAS), and Odom criteria. Adverse events were recorded. Results: Of the identified patients, 83 (45 men, 38 women) patients with a mean age of 53 years participated in the study. Mean follow-up was 3.6 (1.0-6.6) years. A total of 117 segmental levels were fused; 107 levels were plated. No implant fragmentation was seen, but shedding of the implant was noted in 7 patients (8.4\%). Shedding was never seen on initial radiographs; the earliest it was seen was 1.2 years after surgery. There was a trend toward inferior clinical outcomes in patients with metal shedding, with $29 \%$ of patients having fair to poor Odom criteria, compared with $14 \%$ for patients without shedding. Nonfusion rates were also proportionally higher in patients with shedding of the implant (29\% v. 9.2\%). Long-term radiographic follow-up of shed implants (mean $6.6 \mathrm{yr}$; range $2.2-8.5 \mathrm{yr}$ ) show that there were no progressive radiographic changes on sequential radiographs done at least 1 year apart. Conclusion: This study shows that TM implants achieve a fusion rate comparable to that of autograft and allograft, as well as good early clinical and radiologic outcomes with minimal complication rates. Metallic shedding of the implant could be seen in some cases, which likely represents bone remodeling after implantation. This may be a risk factor for nonfusion and suboptimal clinical outcomes.

\section{2}

Arthroplasty versus fusion surgery following anterior decompression - the Canadian experience. Sean Christie, ${ }^{1}$ Raja Rampersaud, ${ }^{2}$ Ken Thomas, ${ }^{3}$ Darren Roffey, ${ }^{4,5}$ Eugene $\mathrm{Wai}^{4,5,6} \mathrm{Kim} \mathrm{Vu} .{ }^{4}$ From the ${ }^{1}$ Division of Neurosurgery, Department of Surgery, Dalhousie University, Halifax, NS; ${ }^{2}$ Division of Orthopaedic Surgery, Toronto Western Hospital, University Health Network, Toronto, Ont.; ${ }^{3}$ Division of Orthopedic Surgery, Department of Surgery, University of Calgary, Calgary, Alta.; ${ }^{4}$ Ottawa Spine Collaborative Analytics Network (OSCAN), Ottawa, Ont.; ${ }^{5}$ Clinical Epidemiology Program, Ottawa Hospital Research Institute, Ottawa, Ont.; ${ }^{6}$ Division of Orthopaedic Surgery, University of Ottawa, The Ottawa Hospital, Ottawa, Ont.

Background: After anterior cervical discectomy, fusion (ACDF) is generally performed. More recently, artificial disc replacement (ADR) is gaining popularity as an alternative. However, there is little Canadian evidence available regarding the benefits of one treatment over the other. Our objective was to determine if outcomes of ADR differed compared with ACDF. Methods: This study was a retrospective multivariate analysis of the prospectively collected national Canadian Spine Outcomes and Research Network (CSORN) database. Multivariate logistic regression was used to adjust for possible confounding effects of age, sex, diagnosis, baseline function and American Society of Anesthesiologists (ASA) score. Results: There were 515 patients identified as being eligible for the study: 39 had ADR in 1 or more spinal levels, and 476 had ACDF. At baseline, the ADR group was significantly younger, had lower ASA grades and tended to be female. There was also a significantly higher proportion of neck pain and a lower proportion of myelopathy in the ADR group. Both ADR and ACDF patients had a similar number of levels operated on, and there were no significant between-group differences at baseline in the percentage of smokers, neck and arm pain scores, overall health score, physical function and mental health scores. No significant differences were identified in surgery time, length of stay or improvements in physical or mental component scores. At 12 months' follow-up, overall improvement in EuroQol visual analogue scale $(0-100)$ for ADR patients was significantly higher (mean 21.7 v. $13.7, p=0.0383$ ), but this difference did not maintain statistical significance in multivariate adjusted analysis. There was also a trend toward lower perioperative adverse events in the ADR group (2.6\% v. $10.1 \%, p=0.1233)$. Conclusion: This is the first Canadian multicentre study to compare outcomes between ADR and ACDF. Although overall improvement in healthrelated quality of life favoured arthroplasty, we were unable to demonstrate this finding following adjusted analysis with our current study numbers.

\section{1}

Use of methylprednisolone for acute spinal cord injury: identification of patterns of practice and reasons for its use worldwide. Alisson R. Teles, ${ }^{1}$ Francine W. Quadros, ${ }^{2}$ Chung Chek Wong, ${ }^{3}$ Giuseppe Barbagallo, ${ }^{4}$ Darrel Brodke, ${ }^{5}$ Abdulaziz Al-Mutair, ${ }^{6}$ K. Daniel Riew, ${ }^{7}$ Asdrubal Falavigna. ${ }^{2}$ From the ${ }^{1}$ Spine Program - University of Calgary, Calgary, Alta.; ${ }^{2}$ Universidade de Caxias do Sul, Caxias do Sul, Brazil; ${ }^{3}$ Sarawak General Hospital, Department of Orthopedic Surgery - Jalan Tun Ahmad Zaidi Adruce, Kuching, Malaysia; ${ }^{4}$ Azienda Ospedaliero - Universitaria Policlinico Neurosurgery, Catania, Italy; ${ }^{5}$ Department of Orthopaedics, University of Utah, Salt Lake City, Utah; ${ }^{6}$ Alrazi Hospital, Ministry of Health/Kuwait Institute for Medical Specialization, Adailiya, Kuwait; ${ }^{7}$ Department of Orthopedic Surgery, Columbia University, New York, NY.

Background: The use of methylprednisolone (MP) in acute spinal cord injury (SCI) is controversial. Recent guidelines do not recommend its routine administration. Our objective was to identify patterns of practice and reasons for MP prescription among spine surgeons worldwide. Methods: Modified versions of a questionnaire published by Hurlbert and Hamilton were used to evaluate opinion about MP administration in SCI. An email request to participate in the survey was sent to members of AOSpine from North America (NA), Latin America (LA), Europe (EU), Asia Pacific (AP) and the Middle East (ME) with a cover letter explaining the objective of this study and an attached link to SurveyMonkey. Bivariate and multivariate analyses were conducted with SPSS. Results: A total of 2659 AOSpine members answered the questionnaire (228 NA, $718 \mathrm{EU}, 972 \mathrm{LA}, 529$ 
$\mathrm{AP}, 212 \mathrm{ME}$ ), at a response rate of $41.5 \%$. Of the total sample, 2064 surgeons $(83 \%)$ reported that they routinely treat patients with acute SCI, and $52.9 \%$ of them reported routine administration of MP for these patients. Multivariate regression showed that use of MP was associated with region (NA, 24\%; EU 47\%; LA, $56 \%$; ME, $62 \%$; AP, $64 \%$; $p<0.001$ ), and number of SCI treated yearly. Only $33 \%$ of surgeons that routinely use MP in acute SCI believe in neurologic improvement with this medication. Reasons for routine administration were different among regions. North American surgeons reported the lowest rate of MP administration by fear of litigation. Steroid use because of fear of litigation was independently associated with orthopedic surgery specialty (adjusted odds ratio 1.55, 95\% confidence interval $1.20-1.99, p<0.001)$. About $63 \%$ of participants reported that their practice on steroid prescription had changed over the past 5 years. Critical appraisal of the current literature was associated with lower rates of MP prescription. Conclusion: Routine administration of MP in SCI is common among spine surgeons. However, most surgeons administer MP for reasons other than belief in clinical benefit. This study identified potential targets for educational campaigns aimed at reducing inappropriate practices of MP administration.

\section{7}

Correlation between magnetic resonance image findings and presence of positive disc cultures in patients undergoing surgery for degenerative disc disease. Husam Almajed, Peter Farzem, Abmed Aoude, Abdulaziz Albajery, Kyle Raasck. From Mcgill University, Montreal, Canada.

Background: The hypothesis that bacterial infection in human intervertebral discs as a plausible cause of degenerative disc disease is remarkable but controversial. The aim of our study was to determine the possible association between disc infection with the development of symptomatic degenerative disc disease and to assess a potential correlation between infected discs and specific magnetic resonance image (MRI) findings. Methods: A retrospective review of all patients undergoing spine surgery for degenerative disc disease was done. Disc biopsies were sent for cultures after gaining consent from patients. After excluding discs that did not meet the criteria and determining the types of organisms in positive discs, samples were categorized for statistical analysis as percentages, and a $\chi^{2}$ test was used to assess statistical significance. Results: We analyzed 91 discs. In total, 20 discs (22\%) were positive for bacteria. A total of $86 \%$ of patients with negative disc cultures had MRIs, whereas $85 \%$ of patients with infected discs had MRIs. Modic changes and Pfirrmann gradings were calculated for both positive and negative discs. For positive discs, $17 \%$ had Modic 1, 76\% had Modic 2 and 5\% had Modic 3. For the negative discs, 3\% had Modic 1, 93\% had Modic 2 and $3 \%$ had Modic 3. For the positive group, $0 \%$ had Pfirrmann 1, $5 \%$ had Pfirrman 2, 35\% had Pfirrman 3, 47\% had Pfirrman 4 and $11 \%$ had Pfirrmann 5 . In the negative group, $0 \%$ had Pfirrmann 1, 9\% had Pfirrmamn 2, 39\% had Pfirrmann 3, 27\% had Pfirrmann 4 and $27 \%$ had Pfirrmann 5. Comparisons showed a positive correlation between positive discs and Modic 1 changes with a $p$ value of less than 0.005 . Conclusion: The link between symptomatic disc degeneration and disc infection is evident. This research also showed a correlation between positive degenerative discs with radiologic findings. Further larger studies are needed to investigate this relationship to warrant the use of prophylactic measures such as antibiotics to delay the degenerative process.

\section{9}

Transition to electronic medical records for preoperative clinical data collection: impact on clinicians' time and administrative costs. Houssam Bouloussa, Niketh Pareek, Andrew Nataraj. From the Department of Surgery, University of Alberta, Edmonton, Alta.

Background: Electronic medical records (EMRs) are promoted as safer, more reliable and more trackable than paper records. The sine qua non condition for the successful implementation of research EMRs is work neutrality for busy clinicians. We therefore hypothesized that the transition from traditional paper-based data collection to EMRs also increases data capture (completeness and timeliness) and helps decrease clinicians' burden while reducing research and administrative costs. Methods: Paperbased preoperative specific forms were prospectively collected from 72 consecutive elective spine patients seen by the same surgeon in February 2017. Following a full transition to an electronic data acquisition system including system alerts for unfilled forms (September 2017), the same data were collected on 66 consecutive patients. The primary outcomes were the proportion and timeliness of complete forms, dedicated time and funds for data collection before and after the transition. A statistical analysis was performed using the Fisher test, $\alpha<5 \%$. Results: Form completion timeliness (same-day filling) was higher in the EMR group (74.2\% v. $23.6 \%, p<0.001)$. In $16.6 \%$ of the paper group, forms were filled in a delayed fashion (mean lag time $142 \mathrm{~d}$ ). Clinician administrative assistants' weekly time dedication to data was significantly lower in the EMR group on the following items: chart organization ( 0 v. $60 \mathrm{~min}$ ) and completion tracking (0 v. $60 \mathrm{~min}$ ). Two hours of research assistant time were saved weekly per surgeon per clinic (estimated \$2760 annual savings). Conclusion: In this nonrandomized single-surgeon study, bias may have affected the outcomes. Patients in the paper group had a high missing data rate owing to lag time exceeding study time. These forms would likely have been filled later, and therefore the proportion of paper forms filled would be much higher. However, contemporaneous data collection enforced by system alerts for unfilled forms may ensure better quality. Automatic form deployment seems to increase data collection timeliness and completion while saving clinicians' and research assistants' time (and therefore funds). A larger scale randomized-controlled study would help clarify potential benefits.

\section{1}

Isolation and characterization of an adult stem cell population within human epidural fat. Nedaa Aljezani, ${ }^{1}$ Roger Cho, ${ }^{1}$ Brian Lenehan, ${ }^{2}$ Roman Krawetz, ${ }^{1}$ Frank Lyons. ${ }^{1}$ From the ${ }^{1}$ University of Calgary, Calgary, Alta.; ${ }^{2}$ University Hospital Limerick and Mater Misericordiae University Hospital, Dublin, Ireland.

Background: To determine if an adult stem cell population resides within human epidural fat $(\mathrm{EF})$ with the ability to differentiate into adipo-, osteo- and chondro-genic lineages. We characterized the self-renewal and differentiation abilities of these 
cells to determine whether they meet the minimal criteria of a mesenchymal stem cell (MSC) as defined by the International Society for Cellular Therapy (ISCT). Mesenchymal stem cells are considered to be an attractive cell source for tissue regeneration (cellular therapy) owing to their chondrogenic, osteogenic and adipogenic capacity. Using the guidelines proposed by the ISCT, we were able to identify EF MSCs in a subset of the patients examined in this study. Methods: Epidural fat cells were obtained and isolated from 10 patients during elective lumbar spine surgery, then digested, cultured and differentiated. Differentiation was analyzed via quantitative polymerase chain reaction and histology. A subset of the cells before differentiation were stained for CD90, CD73, CD44, CD105 and CD271 to examine the cell surface marker profile. Another subset of the cells underwent cell contractility assay. Results: In 3 out of 10 patients examined in this study, the EF-derived cell populations showed multipotent differentiation capacity. These cells expressed cell surface markers consistent with MSCs (CD90+, CD73+, CD44+, low CD105+); however, all cells lacked the expression of CD271. Of note, all cell populations showed robust collagen gel contractility potential. Conclusion: These results show that MSC populations (tri-lineage potential, surface marker expression) were present in $30 \%$ of the patients examined in this study. In the remaining $70 \%$ of patients examined, the EF cells met the definition of a progenitor cell (uni- or bi-potent differentiation capacity with MSC marker expression). Cell populations from all patients also showed myofibroblast-like behaviour in the ability to contract collagen gels. Now that we have confirmed that an MSC progenitor population exists within the $\mathrm{EF}$, it will be critical to characterize the role of these cells in vivo and determine whether it is appropriate to use these cells for regenerative medicine approaches for spine injury or disease.

\section{9}

Divergent configuration improves insertion torque and pullout strength of anterior cervical screws. Fred Xavier, ${ }^{1}$ Natban Cornish, ${ }^{2}$ Rebecca Fason-Rousseau, ${ }^{2}$ Dipal Chatterjee, ${ }^{2}$ Westley Hayes, ${ }^{2}$ Qais Naziri, ${ }^{2}$ Hiroyuki Yoshibara, ${ }^{2}$ and Subrata Saba. ${ }^{2}$ From the ${ }^{1}$ Department of Surgery (Spine), Dalhousie University, Halifax, Nova Scotia, Canada; ${ }^{2}$ Department of Orthopaedic Surgery, SUNY Downstate Medical Center, Brooklyn, USA.

Background: Anterior spinal fusion surgeries such as anterior cervical discectomy and fusion (ACDF) and anterior lumbar interbody fusion (ALIF) are commonly performed with cancellous screws placed a $30^{\circ}$ convergence. However, there is no consensus on the relationship between regional variation in vertebral body bone mineral density (BMD) and screw orientation for optimal stability and fusion. The purpose of this preliminary study was to test the hypothesis that cancellous screws placed in the lateral column of the vertebra offer more resistance to axial pullout than convergent anterocentral screws. Correlation between mechanical strength and density measurements obtained from various techniques was also investigated. Methods: Twelve cervical (C6 and C7), thoracic (T6 and T7) and lumbar (L2 and L3) vertebrae were harvested from 2 fresh human cadavers (40-yr-old man and 32-yr-old woman). Bone mineral density was measured using dual energy X-ray absorptiometry (DEXA), computed tomography (CT), and Faxitron radiography. Each vertebra was caste in epoxy resin, and 2 self-tapping (diameter $4.2 \mathrm{~mm}$ ) cancellous screws of the same length were implanted for side-by-side comparison. One screw was placed at $30^{\circ}$ convergence and another at $10^{\circ}$ divergence. The screw length was measured to be $85 \%$ of the vertebral anteroposterior diameter. The insertion torque was measured using a torque dynamometer. The specimens were then mounted on a mechanical testing machine (Instron) for pullout testing. Results: The central region of the cervical vertebrae was $14 \%$ less dense than the lateral region. Cervical convergent screws mean insertion torque, pullout strength, and stiffness were $58 \mathrm{~N} / \mathrm{cm}, 1094 \mathrm{~N}$ and $654 \mathrm{~N} / \mathrm{mm}$, respectively. Cervical divergent screws showed an increase in torque $(32 \%)$, pullout strength (2\%) and stiffness (10\%). However, the centre was denser and mechanically stronger than the periphery at the thoracic level. Conclusion: These preliminary data showed regional variations in biomechanical properties within the vertebral bodies of young adults. The lateral region of the cervical vertebrae was denser and stronger than the central one. Pullout strength and stability for ACDF could be improved with a divergent screw orientation. The opposite relationship was observed at the thoracic region. We are currently gathering data on the lumbar vertebrae. More vertebrae from older adults will be tested to assess the relationship with aging and osteoporosis.

40

Use of 3 rods to manage large and rigid curves in adolescent idiopathic scoliosis: an alternative technique. Alejandro Peiro-Garcia, ${ }^{1,2}$ Garielle Brown, ${ }^{2}$ Khaled Fawaz, ${ }^{1,2}$ David Parsons, ${ }^{1,2}$ Fabio Ferri-De-Barros. ${ }^{1,2}$ From the ${ }^{1}$ Alberta Children's Hospital, Calgary, Alta.; ${ }^{2}$ University of Calgary, Calgary, Alta.

Background: Spinal arthrodesis to manage adolescent idiopathic scoliosis (AIS) is one of the elective surgical procedures most frequently performed in children's hospitals in North America. Large and rigid curves have been associated with increased complications and health resource utilization. Despite the increased trend to perform osteotomies to manage rigid curves, we propose an alternative novel technique to mitigate the high risk of complications such as spinal cord injury related to this technique. To the best of our knowledge, this is the first study reporting the use of 3 rods to manage severe cases of AIS. Methods: Twenty patients with AIS managed with 3 rods were included in the study. Demographic characteristics, radiographic outcomes, health resource use and complications were reviewed retrospectively with a median follow-up of 2 years. Radiographic outcomes including coronal Cobb angles, coronal curve flexibility, thoracic kyphosis T5-T12 (TK), T2-T5 kyphosis (T2T5), lumbar lordosis (LL), apex vertebral translation (AVT), coronal balance and sagittal balance were reported preoperatively and 2 years postoperatively. Health resource outcome measures included operative time, calculated blood loss, blood transfusion requirement, perioperative complications and cost comparison. Results: Indications for using 3 rods were AIS patients with coronal Cobb angles bigger than $70^{\circ}$ and less than $50 \%$ of correction in the lateral bending radiographs. The median hospital stay was 5 (4-11) days. The median percentage of main Cobb angle correction was $50 \%$ (45\%-62\%). The median of estimated blood loss was $550(350$ $775) \mathrm{mL}$, and median blood transfusion rate was $0 \%(0 \%-2 \%)$. Median operative time was $229(210-254.5)$ min. Median total 
cost per case was CAN\$24097.4 (21348.1-26937.2). The complication rate was $5 \%$ with a superficial wound infection. No neurologic complications or instrumentation bone failures were reported. Conclusion: Although rigid AIS curves have been managed with diverse techniques including aggressive osteotomies, new strategies have to be developed to mitigate the risk of neurologic complications. We present an alternative strategy to decrease these risks in patients with rigid and large curves. According to our results, the use of 3 rods to manage these curves has been shown to improve Cobb angles with a low complication rate. The low mean operative time, short length of stay and low transfusion rates lead to a health resource utilization similar to milder curves. To the best of our knowledge, this is the first work reporting the benefits of this technique.

\section{3}

Spinal casting in severe early onset scoliosis. Fennifer Dermott, Reinhard Zeller. From the Hospital for Sick Children, Toronto, Ont.

Background: The objective of this study was to evaluate the use of spinal casting as a treatment strategy to delay the initiation of growth-friendly surgical techniques in children with severe early onset scoliosis. Methods: This study is a retrospective chart and radiographic review of 13 consecutive patients who underwent spinal casting between July 2014 and September 2017 at a single pediatric tertiary care centre. Spinal casting is a series of 3 casts applied over a 6-month period, with each cast worn for 8 weeks. This is followed by full-time rigid brace wear. Spinal casting is repeated about once a year, with a single cast applied and worn for 2 months. The Cobb angle of the largest curve was measured before the initiation of casting (baseline) and compared with the Cobb angle measurements with the child in-cast, in-brace and out of brace at their most recent follow-up. Independent $t$ tests were used to compare the change in Cobb angle from baseline. Results: Thirteen children ( 7 girls) were reviewed. Of these, 7 were idiopathic, 5 syndromic and 1 neuromuscular. The mean age at initial cast application was 3.5 (1.9-5.6) years. At baseline, the mean Cobb angle was $66.8^{\circ} \pm 17.2^{\circ}\left(\right.$ range $45^{\circ}-91^{\circ}$ ). This improved to $29.3^{\circ} \pm$ $13.1^{\circ}(p<0.001)$ in cast and $39^{\circ} \pm 16.6^{\circ}$ in-brace $(p<0.001)$. At most recent follow-up, the mean Cobb angle was $54.4^{\circ} \pm 17.1^{\circ}(p=$ 0.0016). At a mean follow-up of 24.9 (range 13.4-39.4) months, none of the 13 children showed curve progression beyond baseline. Conclusion: Spinal casting, used in conjunction with full-time rigid bracing, is an effective treatment strategy to control severe early onset scoliosis and to delay the initiation of growth-friendly surgical techniques, which are associated with high complication rates and diminished returns over time.

\section{1}

Variations in density and shear strength within the human thoracic vertebral end plate and trabecular bone. Fred Xavier, ${ }^{1}$ Rebecca Fason-Rousseau, ${ }^{2}$ Dipal Chatterjee, ${ }^{2}$ Westley Hayes, ${ }^{2}$ Subrata Saba. ${ }^{2}$ From the ${ }^{1}$ Department of Surgery (Spine), Dalhousie University, Halifax, NS; ${ }^{2}$ Department of Orthopaedic Surgery, SUNY Downstate Medical Center, Brooklyn, NY.

Background: Previous studies investigated the overall mechanical strength of the vertebral body; however, limited information is available on the biomechanical properties of different regions within the vertebral end plate and cancellous bone. In addition, the correlation between mechanical strength and various density measurements has not been studied yet. This preliminary study proposed to assess the biomechanical strength of different regions of the vertebral body and their role on the overall stability of the human spine. Correlations between shear strength and bone density measured using various techniques were obtained. Methods: Thoracic (T10) vertebrae were harvested from 15 human cadaveric spines (mean age $77 \mathrm{yr}$ ). Twelve cylindrical cores of $7.2 \times$ $3.2 \mathrm{~mm}$ (diameter $\times$ height) were prepared from each vertebral body. Shear was produced using a stainless steel tubular blade and measured with a load cell from a mechanical testing machine (Instron 5566, Instron, Norwood, Massachusetts). Optical and bulk densities were calculated before mechanical testing. Apparent, material and ash densities were measured after testing. Results: Density increased from anterior to lateral regions of both end plate and cancellous bone. Shear strength was significantly lower in the anterior $(0.52 \pm 0.08 \mathrm{MPa})$ than in the lateral region $(2.72 \pm 0.59 \mathrm{MPa})(p=0.017)$. From the inferior to the superior end plates, shear strength and maximum load to failure decreased by $23 \%$ and $33 \%$, respectively $(p>0.05)$. Trabecular bone maximum load carrying capacity was 5 times higher in the lateral $(12 \pm 2.74 \mathrm{~N})(p=0.09)$ and 4.5 times higher in the central $(10 \pm 2.24 \mathrm{~N})(p=0.2)$ than in the anterior $(2 \pm 0.60 \mathrm{~N})$ regions. Mechanical strength positively correlated with ash density and most closely with material density. Conclusion: Shear strength was the lowest at the anterior region and highest at the lateral region for both end plate and cancellous bone. Material density had the best correlation with mechanical strength. Results from this study may explain the vertebral fracture patterns in osteoporotic patients. Furthermore, newer spinal implants could optimize the loading in the lateral aspects of both end plate and cancellous bone; thus, reducing the likelihood of screw loosening and the subsidence of disc replacement devices. However, owing to a small sample size, our study was underpowered. We are currently testing more specimens.

66

One year results of a prospective, multicenter study to assess the clinical efficacy and fusion rates of trabecular metal interbody instrumented lumbar fusion. Husam Almajed, ${ }^{1}$ Abmed Aoude, ${ }^{1}$ Rune Hedlund, ${ }^{1}$ Förg Franke, ${ }^{1}$ Geoffrey Lesage, ${ }^{1}$ Luis A Galovich, ${ }^{1}$ Patrick Chatellier, ${ }^{1}$ Fan Splawinski, ${ }^{2}$ Gunter Raderschadt, ${ }^{1}$ Albert Yee, ${ }^{1}$ Peter Farzem. ${ }^{1}$ From the ${ }^{1}$ McGill University Health Center, Montréal, Que.; ${ }^{2}$ Vernon Jubilee Hospital, Vernon, BC.

Background: Trabecular metal has been used in orthopedics for many years with positive effects in total hip arthroplasty. In this study, we investigate the rate of radiographic fusion and clinical outcomes for patients treated with a trabecular metal interbody fusion system. Methods: A multicentre prospective study was completed with 77 patients treated with a new trabecular metal interbody fusion system. Radiologic assessment of all patients was completed at 3, 6, 12 and 24 months. An adequate fusion was defined as less than $50 \%$ radiolucency on radiographs at any time. Clinical outcome was also evaluated with Oswestry Disability Index (ODI) and EuroQOL 5-Dimension (EQ5D) questionnaires at the 3,6 and 12 months. Results: Radiographic results 
showed a $100 \%$ fusion rate at 6,12 and 24 months, with only $2 \%$ showing nonfusion at the 3 -month mark. Patient ODI scores showed statistically significant decreases $(p<0.01)$ at all followups (3, 6 and $12 \mathrm{mo})$. Preoperative ODI was a mean of 56.7 (95\% confidence interval [CI] 53.4-59.9) compared with ODIs of 31.7 (95\% CI 27.3-36.1), 32.7 (95\% CI 27.5-37.8) and 29.7 (95\% CI 24.5-34.8) for 3, 6 and 12 months, respectively. In addition, a decrease of more than 15 points in the ODI was seen in $68 \%$ of patients at the 12-month mark. Similarly, EQ5D scores all increased with statistical significance $(p<0.001)$ at all follow-ups. Mean preoperative EQ5D was 33.6 (95\% CI 25.5-38.6) compared with 58.1 (95\% CI 51.3-65.0), 61.0 (95\% CI 54.6-67.5) and 60.8 (95\% CI 53.6-68.0) for 3, 6 and 12 months, respectively. Conclusion: Our study demonstrates good radiologic outcome of a new trabecular metal interbody fusion system, with $100 \%$ of patients showing signs of fusion at the 6-month mark. In addition, clinical outcomes were favourable up to 12 months for both ODI and EQ5D scores in comparison to preoperative values. Therefore, this study shows promising radiographic and clinical results for a new trabecular metal interbody fusion system.

\section{2}

Utility and reliability of magnetic resonance imaging classifications for predicting lumbar arthroplasty outcomes. Khaled Almansoori, ${ }^{1}$ Godefroy Hardy-St.Pierre, ${ }^{2}$ Facques Bouchard. ${ }^{2}$ From the ${ }^{1}$ Sheikh Khalifa Medical City, Abu Dhabi, United Arab Emirates; ${ }^{2}$ University of Calgary, Calgary, Alta.

Background: Clinical outcomes of lumbar total disc replacements (TDR) depend on multiple factors, including the preoperative condition of the vertebrae and facet joints. Several studies have highlighted magnetic resonance imaging (MRI) as the modality of choice for evaluating these features, but none have compared their utility or reliability in predicting clinical outcomes following single-level lumbar arthroplasty procedures. Methods: A retrospective observational cohort study was performed by evaluating clinical outcomes among patients undergoing single-level lumbar TDR procedures. In addition to revision and reoperation rates, patients who met inclusion criteria were asked to complete a visual analog scale (VAS) pain survey, the Oswestry Disability Index (ODI) questionnaire and a general satisfaction survey. These outcomes were then evaluated in relation to 9 lumbar MRI classifications schemes: Modic, modified Modic, Schneiderman, modified Schneiderman, Pfirrmann, modified Pfirrmann, modified Tufts Score, Weishaupt and Grogan. Pearson correlation, Cohen $\kappa$ coefficient and binomial regression analyses were performed using SPSS version 22 (SPSS Inc., Chicago, Illinois). Results: A total of 45 lumbar TDR were included with a mean follow-up of 7.8 years. The inter- and intra-observer reliabilities were generally fair to excellent, depending on the classification schemes $(\kappa=0.27-$ 0.78 and $\kappa=0.36-0.84$, respectively). Correlation analysis showed poor-to-moderate relations between MRI grading and ODI scores $\left(R^{2}=-0.01\right.$ to -0.32$)$, VAS leg scores $\left(R^{2}=-0.04\right.$ to $-0.41)$, VAS back scores $\left(R^{2}=-0.05\right.$ to -0.35$)$ and outcome satisfaction $\left(R^{2}=-0.01\right.$ to -0.36$)$. Modic and modified Schneidermann classifications were the most reliable classifications in terms of agreement reliability and outcome predictions, whereas Weishaupt and Grogan classifications were the least reliable. Conclusion: The ability of MRI classifications to predict clinical outcomes among single-level lumbar arthroplasty procedures is generally moderate, with leg pain resolution and ODI improvements being the most and least predictable outcomes, respectively. Reliability of intravertebral disc grading is generally fair to excellent, whereas facet-joint grading is fair at best. Overall, Modic and modified Schneidermann were the most useful and reliable classification systems.

\section{3}

Off-hours surgery for spinal metastasis. Amer Sebaaly, ${ }^{1}$ Zhi Wang, ${ }^{1}$ Daniel Shedid, ${ }^{2}$ Ghassan Boubez. ${ }^{1}$ From the ${ }^{1}$ Department of Orthopedics, Centre Hospitalier de l'Université de Montréal, Montreal, Que.; ${ }^{2}$ Department of Neurosurgery, Centre Hospitalier de l'Université de Montréal, Montreal, Que.

Background: Off-hours surgery in orthopedic surgery, particularly in spine surgery, is associated with increased complications. There is no study in the literature evaluating the effect of surgical timing on complication and outcomes of spinal metastasis. The objective of this study was to evaluate the complications and outcomes of off-hours spinal metastasis surgery. Methods: This is a retrospective analysis of prospectively collected data. Preoperative, operative and postoperative data were collected in addition to complications and Frankel score at all time checkpoints. Off-hours surgery was defined as surgery starting between 17:00 and 08:00 the following day, or surgery performed during the weekend. We set $p$ less than 0.05 as the significance threshold. Results: We included 376 patients, with an incidence of offhours surgery of $32 \%$. There was increased neurologic compromise and sphincteric dysfunction in the off-hours group. Higher American Society of Anesthesiologists (ASA) score and older age were increased in the off-hours group. There was decreased operative bleeding with off-hours surgery, with no difference in bleeding and number of fixed vertebrae. There was a higher percentage of neurologic improvement with off-hours surgery compared with in-hours surgery. Finally, there was no effect on surgical timing on patient survival. Conclusion: This is the first report of the effect of off-hours surgery on complications and outcomes of spinal metastasis. Greater neurologic compromise and higher age and ASA scores were associated with higher incidence of off-hours surgery. Off-hours surgery is associated with decreased surgical time and higher percentage of neurologic improvement. Finally, there is no effect of surgical timing on survival rates. 